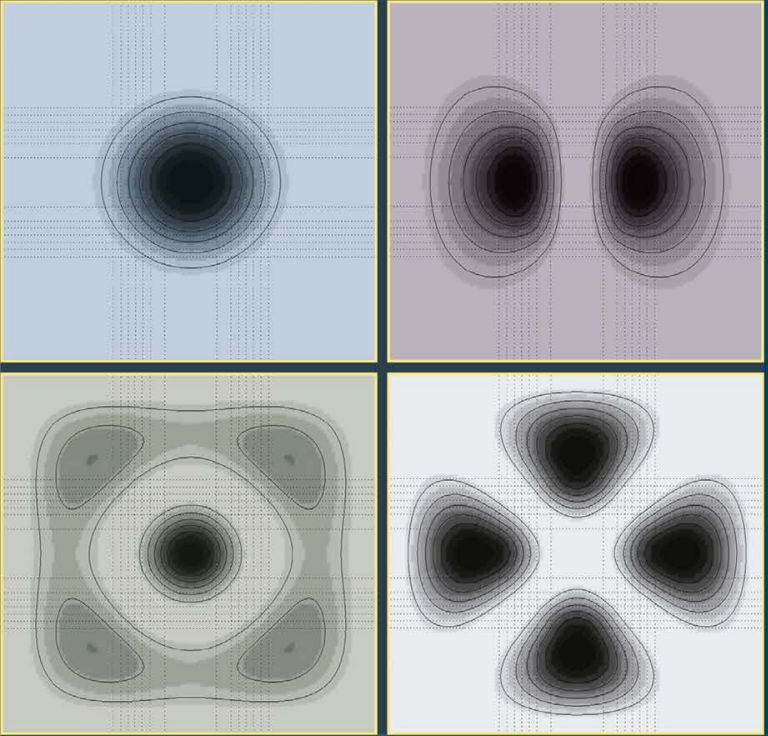

\title{
Diffractive Optics and
}

Nanophotonics

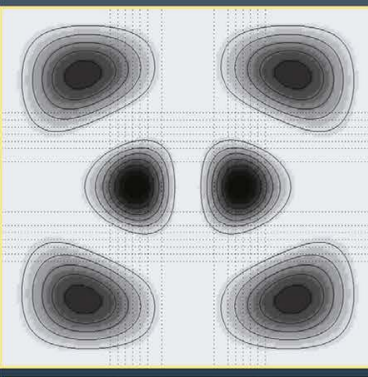

\begin{tabular}{lll}
\hline 0 & 0 \\
\hline
\end{tabular}

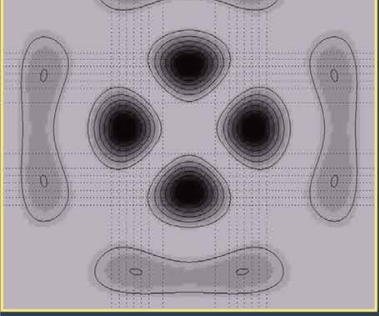

6
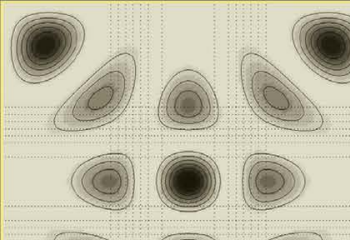

(ए)
Edited by V. A. Soifer
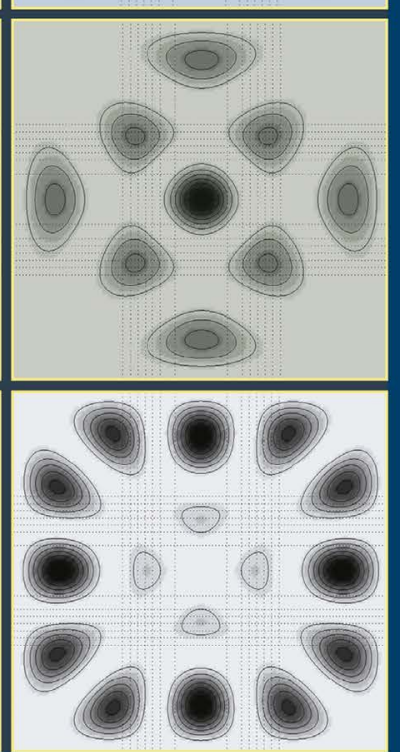


\section{Diffractive Optics and Nanophotonics}




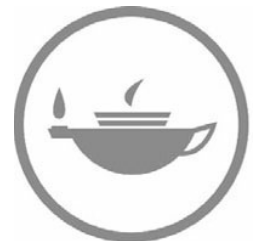

Taylor \& Francis Taylor \& Francis Group

http://taylorandfrancis.com 


\title{
Diffractive Optics and Nanophotonics
}

\author{
Edited by \\ V. A. Soifer
}

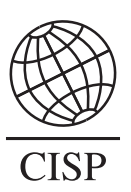

(CRC

CRC Press

Taylor \& Francis Group

Boca Raton London New York

CRC Press is an imprint of the

Taylor \& Francis Group, an informa business 


\section{CRC Press}

Taylor \& Francis Group

6000 Broken Sound Parkway NW, Suite 300

Boca Raton, FL 33487-2742

\section{(c) 2017 by CISP}

CRC Press is an imprint of Taylor \& Francis Group, an Informa business

No claim to original U.S. Government works

Printed on acid-free paper

International Standard Book Number-13: 978-1-4987-5447-7 (Hardback)

This book contains information obtained from authentic and highly regarded sources. Reasonable efforts have been made to publish reliable data and information, but the author and publisher cannot assume responsibility for the validity of all materials or the consequences of their use. The authors and publishers have attempted to trace the copyright holders of all material reproduced in this publication and apologize to copyright holders if permission to publish in this form has not been obtained. If any copyright material has not been acknowledged please write and let us know so we may rectify in any future reprint.

Except as permitted under U.S. Copyright Law, no part of this book may be reprinted, reproduced, transmitted, or utilized in any form by any electronic, mechanical, or other means, now known or hereafter invented, including photocopying, microfilming, and recording, or in any information storage or retrieval system, without written permission from the publishers.

For permission to photocopy or use material electronically from this work, please access www.copyright. com (http://www.copyright.com/) or contact the Copyright Clearance Center, Inc. (CCC), 222 Rosewood Drive, Danvers, MA 01923, 978-750-8400. CCC is a not-for-profit organization that provides licenses and registration for a variety of users. For organizations that have been granted a photocopy license by the CCC, a separate system of payment has been arranged.

Trademark Notice: Product or corporate names may be trademarks or registered trademarks, and are used only for identification and explanation without intent to infringe.

Visit the Taylor \& Francis Web site at

http://www.taylorandfrancis.com

and the CRC Press Web site at

http://www.crcpress.com 


\section{Contents}

1. The Fourier modal method and its use in plasmonics and the theory of resonant diffraction gratings

1.1. The Fourier modal method of solving the diffraction problems 3

1.1.1. The equation of a plane wave 3

1.1.2. The Fourier modal method for two-dimensional periodic $\begin{array}{ll}\text { structures } & 7\end{array}$

1.1.2.1. The geometry of the structure and formulation of the problem 8

1.1.2.2. Description of the field above and below the structure 9

1.1.2.3. The system of differential equations for the description of $\begin{array}{ll}\text { the field in the layer } & 10\end{array}$

1.1.2.4. Representation of the field inside the layer 21

1.1.2.5. 'Stitching' of the electromagnetic field at the boundaries of $\begin{array}{ll}\text { layers } & 23\end{array}$

$\begin{array}{ll}\text { 1.1.2.6. The scattering matrix algorithm } 26 & 23\end{array}$

1.1.2.7. Calculation of the field distribution 30

1.1.2.8. Intensities of the diffraction orders 31

1.1.2.9. Numerical example 33

1.1.3. The Fourier modal method for three-dimensional

1.1.4. The Fourier modal method for two-dimensional non-

1.1.4.1. The geometry of the structure and formulation of the problem 40

1.1.4.2. Perfectly matched absorbing layers 41

1.1.4.3. Solution of the diffraction problem 52

1.1.4.4 The energy characteristics of the reflected and transmitted light 54

1.1.4.5. Numerical example $\quad 55$

1.2. Methods for calculating eigenmodes of periodic diffractive
structures

1.2.1. Calculation of modes based on the calculation of the poles of the scattering matrix $\quad 56$

1.2.1.1. Resonant representation of the scattering matrix $\quad 57$

$\begin{array}{ll}\text { 1.2.1.2. Calculation of the poles of the scattering matrix } & 60\end{array}$ 
1.2.1.3. Numerical examples

1.2.2. Calculation of events based on calculating eigenvalues of the transfer matrix

1.2.2.1. Calculation of modes

1.2.2.2. Calculation of the field distribution 75

1.2.2.3. Numerical example 78

1.3. Resonant diffraction gratings for transformation of optical pulses

1.3.1. The integration and differentiation of optical pulses.

Wood's anomalies

1.3.2. Types of gratings for integration and differentiation of pulses

1.3.3. Fractional integration and differentiation of optical pulses. Rayleigh-Wood anomalies $\quad 84$

1.3.4. Examples of calculation of pulse differentiation 86

1.3.4.1. Calculation of the grating for differentiating pulses 86

1.3.4.2. Calculation of the grating for fractional differentiation of pulses

1.3.5. Example of calculation of the integrating gratings 88

1.3.5.1. Calculation of the grating for the integration of pulses 88

1.3.5.2. Calculation of the grating for the fractional integration of pulses

1.4. Diffractive optical elements for controlling the propagation of surface plasmon-polaritons

1.4.1. The dispersion relation and the properties of surface plasmon-polaritons

1.4.1.1. Dispersion relations 92

1.4.1.2. The properties of surface plasmon-polaritons 101

$\begin{array}{ll}\text { 1.4.1.3. Plasmonic waveguides } & 104\end{array}$

1.4.1.4. Excitation of surface plasmon-polaritons 106

1.4.2. Phase modulation of surface plasmon-polaritons by
dielectric structures

1.4.3. Suppression of parasitic scattering of surface plasmonpolaritons

1.4.3.1. Theoretical analysis of matching of transverse profiles of the field of the surface plasmon-polariton and the plasmonic mode of the dielectric/dielectric/metal waveguide

1.4.3.2. Suppression of parasitic scattering during the passage of surface plasmon-polaritons through two-layer dielectric structures

1.4.4. Design of the lens for focusing the surface plasmonpolaritons

1.4.4.1. Integral representations of the field in the form of the spectrum of surface plasmon-polaritons

1.4.4.2. Diffraction lenses for focusing the surface plasmon- 
polaritons

1.4.4.3. The calculation of diffractive lenses for surface plasmon polaritons with parasitic scattering suppression

1.4.5. Calculation of reflective Bragg gratings for surface plasmon-polaritons

1.5. Generation of interference patterns of evanescent electromagnetic waves by means of diffraction gratings

1.5.1. Theoretical description of the interference patterns of evanescent diffraction orders of gratings with one-dimensional and two-dimensional periodicity

1.5.1.1. One-dimensional interference patterns

1.5.1.2. Two-dimensional interference patterns

1.5.2. Formation of interference patterns of evanescent electromagnetic waves in the metal-dielectric diffraction gratings

1.5.2.1. Generation of one-dimensional interference patterns 146

1.5.2.2. Controlling the period of one-dimensional interference patterns by changing the parameters of the incident wave

1.5.2.3. Generation of two-dimensional interference patterns and control of their shape by changing the parameters of the incident wave

1.5.3. Generation of interference patterns of evanescent electromagnetic waves in dielectric diffraction gratings

1.5.3.1. Generation of one-dimensional interference patterns

1.5.3.2. Generation of two-dimensional interference patterns

2. Components of photonic crystals

2.1 One-dimensional and two-dimensional photonic crystals

2.1.1 Photonic bandgaps

2.1.2. Diffraction of a plane wave on a photonic crystal with no defects

2.1.3. The propagation of light in a photonic crystal waveguide

2.1.4. Photonic crystal collimators 188

2.2. The two-dimensional photonic crystal gradient Mikaelian lens 189

2.2.1. The modal solution for a gradient secant medium 191

2.2.2. Photonic crystal gradient lenses 193

2.2.3. Photonic crystal lens for coupling two waveguides 198

2.3. Simulation of three-dimensional nanophotonic devices for input of radiation in a planar waveguide

2.3.1. The two-dimensional simulation of the input of light in a planar waveguide using a grating

2.3.2. Three-dimensional modelling Mikaelian photonic crystal lens for coupling two waveguides

2.3.3. Three-dimensional modeling of the entire nanophotonic device 
2.4. Photonic crystal fibres

2.4.1. Calculation of modes of photonic-crystal fibres by the method of matched sinusoidal modes (MSM method)

2.4.2. Method of matched sinusoidal modes in the vector case

2.4.3. Krylov method for solving non-linear eigenvalue problems

2.4.4. Calculation of stepped fibre modes

2.4.5. Calculation of modes of photonic crystal fibres 256

2.4.6. Calculation of modes using Fimmwave

3. Sharp focusing of light with microoptics components

3.1. Numerical and experimental methods for studying sharp focusing of light

3.1.1. BOR-FDTD method

3.1.2. Richards-Wolf vector formula 284

3.1.3. Scanning near-field optical microscopy 291

3.2. Axicon 292

3.2.1. Sharp focusing of light with radial polarization using a microaxicon

3.2.2. Diffractive logarithmic microaxicon 303

3.2.3. Binary axicons with period 4, 6 and $8 \mu \mathrm{m} \quad 312$

$\begin{array}{lll}3.2 .4 \text {. Binary axicon with a period of } 800 \mathrm{~nm} & 327\end{array}$

3.3. Fresnel zone plate $\quad 330$

3.3.1. Comparison with the Richards-Wolf formulas 331

3.3.2. Symmetry of the intensity and power flow of the
subwavelength focal spot

3.4. Focusing light with gradient lenses 348

3.4.1. Mechanism of superresolution in a planar hyperbolic secant lens

3.4.2. Gradient elements of micro-optics for achieving superresolution

3.4.3. Construction of an enlarged image with superresolution using planar Mikaelian lenses

3.4.4. Hyperbolic secant lens with a slit for subwavelength focusing of light

3.4.5. Sharp focusing of radially polarized light with a 3D hyperbolic secant lens

3.4.6. Optimizing the parameters of the planar binary lens for the visible radiation range

3.5. Formation of a photon nanojet by a sphere 402

3.5.1. Numerical simulation of passage of continuous radiation through the microsphere

3.5.2. Numerical simulation of passage of pulsed radiation through a microsphere 
4. Introduction

4.1. Formation of vortex laser beams by using singular optics elements

4.1.1. Theoretical analysis of the diffraction of plane and Gaussian waves on the spiral phase plate

4.1.2. Theoretical analysis of the diffraction of plane and Gaussian waves on a spiral axicon

4.1.3. Numerical modeling of the diffraction of different beams on elements of singular optics

4.1.4. Conclusions

4.2. Vector representation of the field in the focal region for the vortex transmission function

4.2.1. Uniformly polarized beams (linear and circular polarization)

4.2.1.1. Linear x-polarization

4.2.1.2. Circular polarization

4.2.2. Cylindrical vector beams (radial and azimuthal polarization)

4.2.2.1. The radial polarization

456

4.2.2.2. The azimuthal polarization

4.2.3. Generalized vortex polarization

4.2.4. Conclusions

4.3. Application of axicons in high-aperture focusing systems

4.3.1. Adding the axicon to the lens: the paraxial scalar model

4.3.2. Apodization of the short-focus lens by the axicon: the non-paraxial vector model in the Debye approximation

4.3.3. Using vortex axicons for spatial redistribution of components of the electric field in the focal region

4.3.4. Conclusions

4.4. Control of contributions of components of the vector electric field at the focus of the high-aperture lens using binary phase structures

4.4.1. Maximizing the longitudinal component for linearly polarized radiation

4.4.2. Increasing efficiency by focusing the radially polarized radiation

4.4.3. Overcoming of the diffraction limit for azimuthal polarization by the transverse components of the electric field

4.4.4. Conclusions

4.5. Minimizing the size of the light or shadow focal spot with controlled growth of the side lobes

4.5.1. Scalar diffraction limit: theoretical analysis

4.5.2. Optimization of the transmission function of the focusing system

4.5.3. Minimization of the light spot for radially polarized radiation 
4.5.4. Formation of light rings of the subwavelength radius azimuthal polarization of laser radiation

5. Optical trapping and manipulation of micro- and nano-objects 519

5.1. Calculation of the force acting on the micro-object by a focused laser beam

5.1.1. Electromagnetic force for the three-dimensional case

5.1.2. Electromagnetic force for the two-dimensional case

5.1.3. Calculation of force for a plane wave

5.1.4. Calculation of force for a non-paraxial Gaussian beam

5.1.5. Calculation of forces for the refractive index of the object smaller than the refractive index of the medium

5.2. Methods for calculating the torque acting on a micro-object by a focused laser beam

5.2.1. The orbital angular momentum in cylindrical microparticles

5.2.2. The results of numerical simulation of the torque

5.1.7. A geometrical optics method for calculating the force acting by light on a microscopic object

5.1.8. Comparison of results of calculations by geometrical optics and electromagnetic methods

5.2. Rotation of micro-objects in a Bessel beam

5.2.1. Rotation of micro-objects in Bessel beams

5.2.2. Optical rotation using a multiorder spiral phase plate

5.2.3. Rotation of microscopic objects in a vortex light ring formed by an axicon

5.2.4. Optical rotation in a double light ring

5.2.5. Formation of the DOE with a liquid-crystal display

5.2.5. Rotation of micro-objects by means of hypergeometric beams and beams that do not have the orbital angular momentum using the spatial light modulator

5.2.6. Quantitative investigation of rotation of micro-objects in light beams with an orbital angular momentum

5.3. Rotating microparticles in light beams designed and formed to obtain the maximum torque

5.3.1. Formation of the light beams, consisting of several light rings

5.3.2. Formation of the superposition of high-order optical vortices

5.3.3. Experimental formation of vortex beams superpositions

5.4. Light beams specially formed for linear displacement and positioning of micro-objects

5.4.1. Encoding of amplitude by the local phase jump method 608

5.4.2. Modification of the method of encoding the amplitude for the calculation of light fields with a complex structure 
5.4.3. Positioning of micro-objects using focusators

5.4.4. Focusators to move micro-objects along predetermined paths $\quad 619$

5.4.5. Focusators for sorting and filtering micro-objects 624

5.4.6. Focusators to filter certain micro-objects 626

5.4.8. The linear movement of micro-objects in superposition of two vortex beams

5.5. Formation of arrays of light 'bottles' with the DOE 642

5.5.1. Bessel light beams and their remarkable properties 644

5.5.2. Multimode Bessel beams 645

5.5.3. Formation of light 'bottles' through the use of composite Bessel beams of $0^{\text {th }}$ order 647

5.5.4. Algorithm for increasing the uniformity of light traps formed on the basis of the gradient procedure

5.5.5. Formation of arrays of light 'bottles'

5.5.6. Formation of arrays of hollow light beams

5.5.7. Experimental formation of arrays of light 'bottles' with the binary DOE

5.5.8. Capture of transparent micro-objects in the system of light 'bottle'

5.5.9. Capture and displacement of metallic tin microparticles with the shape close to spherical

5.5.10. The deposition and positioning of micro-objects using arrays of hollow light beams

5.6. The light beams generated by the DOE for non-damaging capture of biological micro-objects

5.6.1. Modification of the Gaussian beam to optimize the power characteristics of the optical trap

5.6.2 Measurements of the energy efficiency of the DOE forming beams-crescents

5.6.3. Experiments with optical manipulation of cells of Saccharomyces cerevisiae 


\section{Introduction}

The phenomenon of diffraction, which was originally seen as a limiting factor in optics, is now a fundamental basis for the creation of a new component base and advanced information technology.

The development of diffractive optics and nanophotonics devices is based on a computer solution of direct and inverse problems of diffraction theory, based on Maxwell's equations. Among the numerical methods for solving Maxwell's equations most widely used are: the finite-difference time-domain method, the finite element method and the Fourier modal method, and also approximate methods for calculating diffraction integrals.

The book is devoted to modern achievements of diffractive optics, focused on the development of new components and devices for nanophotonics, and devices and information technologies based on them.

The first chapter describes the Fourier modal method, designed for the numerical solution of Maxwell's equations, as well as some of its applications in problems of calculation of diffractive gratings with the resonance properties and plasmon optics components.

The Fourier modal method (or rigorous-coupled wave analysis) has a wide range of applications. In the standard formulation the method is used to solve the problems of diffraction of a monochromatic plane wave on diffraction gratings. Introduction of the light beam in a plane-wave basis allows to use the method for modelling the diffraction of optical pulses. Using the so-called perfectly matched layers in combination with artificial periodization enables the method to be used efficiently to solve the problems of the diffraction of light waves by non-periodic structures. In this chapter the Fourier modal method is considered for solving the diffraction of a plane wave in the two-dimensional and three-dimensional diffractive gratings, as well as in the case of non-periodic structures. The implementation of the method is based on the numerical-stable approach, known as the scattering matrix method. 
The resonance features in the spectra of periodic diffraction structures are studied using the methods developed by the authors for calculating the scattering matrix poles. These methods take into account the form of the matrix in the vicinity of the resonances associated with the excitation of eigenmodes in the lattice and, compared with the known methods, have better convergence.

The Fourier modal method and scattering matrix formalism are applied to the calculation of diffractive gratings with the resonance properties for the conversion of optical pulses. The chapter proposes a theoretical model of resonant gratings performing operations of differentiation and integration of the optical pulse envelope and the results of the calculation and research of diffractive gratings for the differentiation and integration of picosecond pulses are presented.

The non-periodic variant of the Fourier modal method is used in the problem of calculating the diffractive optical elements for controlling the propagation of surface plasmon-polaritons. The principle of operation is based on the phase modulation of surface plasmon-polaritons by dielectric steps with changing height and length and located on the surface of the metal.

The Fourier modal method is also applied to the task of calculating the diffractive gratings forming, in the near-field, interference patterns of evanescent electromagnetic waves and, in particular plasmon modes. The chapter provides a theoretical description and a number of numerical examples of calculation of the gratings forming interference patterns of evanescent electromagnetic waves and plasmon modes with a substantially subwavelength period and demonstrates the ability to control the type and period of the interference patterns of damped waves due to changes in the parameters of the incident radiation.

The practical use of the results of the first chapter includes systems for optical computing and ultra-fast optical information processing, the creation of high-performance components plasmon optics, the contact lithography systems and the systems for optical trapping and manipulation of nanoscale objects.

The second chapter deals with the nanophotonics components based on photonic crystals: the gradient planar photonic crystal (PC) lens and photonic crystal fibers. The ultra-compact nanophotonic device is described for effectively connecting two-dimensional waveguides of different widths using the PC-lens. It is shown that the PC-lens focuses the light into a small focal spot directly behind the lens whose size is substantially smaller than the scalar diffraction 
limit. The simulation was performed using a finite difference solution of Maxwell's equations.

The second chapter also describes the method of calculation of optical fibres with the PC cladding. In this waveguide the light propagates within the core not due to the effect of total internal reflection from the core-cladding interface, and by reflection from a multilayer Bragg mirror formed by the system of periodically spaced holes around the core. Calculation of spatial modes in PC-fibres is based on partitioning the inhomogeneous fibre cross-section into a set of rectangular cells, each with a set of known spatial sinusoidal modes. Further cross-linking of all modes is carried out at the interfaces of all cells. The PC waveguides differ from the step and gradient waveguides by that they allow the modes to be localized within the core, that is all the modes propagate inside the core and almost do not penetrate into the cladding thus increasing the diameter of the mode localized within the core. In addition, the PC waveguides with a hollow core help to avoid chromatic dispersion in the fibre and transmit light with higher power. A short pulse of light passing through in a PC waveguide of finite size is transformed at the output to white light due to non-linear dispersion. Sections of the PC waveguides are used as filters, white light sources, and non-linear optics for second harmonic generation.

The third chapter discusses the focusing of laser radiation. The concept of the diffraction limit was established in the 19th century: $d_{\min }=\lambda /(2 n)$, where $\lambda$ is the wavelength of light in vacuum, $n$ is the refractive index of the medium. The third chapter shows that using diffractive micro-optics components, focusing the light near their surface it is possible to overcome the diffraction limit. Attention is given to the sharp focusing of laser light using micro-components such as the axicon, the zone plate, the binary and gradient planar microlens, microspheres. Focusing light near the surface of the microcomponents allows to overcome the diffraction limit as a result of the presence of surface waves and the influence of the refractive index of the material of the focusing element. Simulation of focusing the laser beam is carried out by the approximate Richards-Wolf vector method and the finite difference solution of Maxwell's equations.

Reducing the size of the focal spot and overcoming the diffraction limit is an urgent task in the near-field microscopy, optical micromanipulation, contact photolithography, increasing the density of recording information on an optical disc, and coupling planar waveguides of different widths. 
The fourth chapter describes the focusing of singular vortex laser beams. At the point of singularity the intensity of the light field is zero, and the phase is not defined. There are abrupt phase changes in the vicinity of this point. Singularities in light fields can appear as they pass through randomly inhomogeneous and nonlinear media. It is also possible to excite vortex fields in laser resonators and multimode optical fibres. The most effective method of forming the vortex laser beams is to use spiral diffractive optical elements, including spiral phase plates and spiral axicons. The fourth chapter discusses the formation of vortex beams represented as a superposition of Bessel, Laguerre-Gauss, Hermite-Gauss, etc. modes. When focusing the vortex beams attention is paid to the combination of different types of polarization and phase singularities which lead to overcoming the diffraction limit of the far-field diffraction zone.

Main applications of vortex laser beams are sharp focusing of laser light, manipulation of microscopic objects and multiplexing the channels of information transmission.

In the fifth chapter we consider the problem of optical trapping, rotation, moving, positioning of micro-objects through the use of diffractive optical elements. Micro-objects are rotated by light beams with an orbital angular momentum. Considerable attention is paid to the methods of calculating the forces acting on the micro-objects in light fields. The problem of creating the torque in micromechanical systems using light beams has a fairly long history. In a number of studied the problem of rotation is considered in conjunction with other tasks: sorting, moving, positioning, etc. It should be noted that in all the above cases the focus is primarily on the manufacturing technology of micromechanics elements and no attempts are made to improve the light beams. At the same time, the calculation and application of diffractive optical elements, forming the vortex light beams for a specific form of the micromechanical component can improve the transmission efficiency of the torque in micromechanical systems.

This chapter discusses two methods of calculating the diffractive optical elements for forming light fields with a given amplitude-phase distribution. One of them is based on calculating a focusator forming a light field with a predetermined phase gradient along the contour. Another method uses the superposition of zero-order Bessel beams to form light traps in the form of hollow beams for opaque microscopic objects. The results of experiments on optical trapping and relocation 
of micro-objects are presented. The chapter examines the possibility of using light beams to move the biological micro-objects.

The book has been written by experts of the Image Processing Systems Institute, Russian Academy of Sciences. In the first chapter, sections 1.1 and 1.2 were written by D.A. Bykov, E.A. Bezus and L.L. Doskolovich, section 1.3 by D.A. Bykov, L.L. Doskolovich and V.A. Soifer, sections 1.4 and 1.5 by E.A. Bezus and L.L. Doskolovich. The second chapter was written by V.V. Kotlyar, A.A. Kovalev, A.G. Nalimov and V.A. Soifer. The third chapter by V.V. Kotlyar, A.A. Kovalev, A.G. Nalimov and S.S. Stafeev. The fourth chapter was written by S.N. Khonina and the fifth chapter by R.V. Skidanov, A.P. Porfir'ev and V.A. Soifer. 


\section{The Fourier modal method and its use in plasmonics and the theory of resonant diffraction gratings}

This chapter describes the Fourier modal method (also called rigorous coupled-wave analysis) designed for the numerical solution of Maxwell's equations, as well as some of its applications in problems of diffractive nanophotonics.

The Fourier modal method has a wide range of applications. The formulation of the method is used for the solution of the problem of diffraction of a monochromatic plane wave on diffraction gratings. The representation of a light beam in the plane wave basis allows to use the method for the simulation of the diffraction of spatiotemporal optical signals (optical pulses). The use of the so-called perfectly matched absorbing layers in combination with artificial periodization enables the method to be used to solve the problems of the diffraction of light waves by non-periodic structures.

In section 1.1 the Fourier modal method is considered in the standard formulation for solving the problem of diffraction of a plane wave on two-dimensional and three-dimensional multilayered periodic diffraction structures and for the case of non-periodic structures. The considered implementation of the method is based on a numerically stable approach, known as the scattering matrix method. The method is described in detail and was used by the authors to create original computer programs for the solution of problems of diffraction on structures of various types. The readers 
can safely use the formulas given in section 1.1 when creating their own programs for electromagnetic modelling.

The interaction of light with a periodic structure, in particular with metal-dielectric structures, is the subject of intense research. In such structures there is a wide range of extraordinary (resonant) optical effects, including extraordinary transmission, total absorption of incident radiation, resonant changes of the transmission and reflection coefficients, the formation of regions with a high degree of localization of energy due to interference of the evanescent and plasmon waves. These effects are usually associated with the excitation in a periodic structure of quasi-guided and plasmonic eigenmodes. The modes of the structure correspond to the poles of the scattering matrix. Section 1.2 presents a number of methods proposed by the authors for calculating the poles of the scattering matrix. These methods take into account the representation of scattering matrix in the vicinity of resonances and, compared with the known techniques, have better convergence.

In section 1.3 the method and the scattering matrix technique are applied to the calculation of diffraction gratings with the resonant properties to transform optical pulses. The first part of this section describes in a common approach a class of transformations of optical pulses realized by resonant diffraction gratings, and proposes a theoretical model of resonant gratings performing operations of differentiation and integration of the optical pulse envelope. The model is based on the resonant representations of the complex amplitude of the zeroth diffraction order in the vicinity of the frequencies corresponding to the waveguide resonances (Wood's anomalies) and in the vicinity of the frequency of the Rayleigh-Wood's anomalies associated with the emergence of propagating diffraction orders. The section also presents the results of calculations and studies of diffraction gratings to perform differentiation and integration of picosecond pulses (Wood's anomalies), as well as the operations of fractional differentiation and integration (Rayleigh-Wood anomalies).

The possible applications of the results of this section include optical computing and ultrafast optical information processing.

In section 1.4 the aperiodic Fourier modal method is used for the design of diffractive optical elements (DOE) to control the propagation of surface plasmon-polaritons (SPP). The principle of operation of the conventional DOE is based on the phase modulation of the input wave field by a diffraction microrelief of variable height. 
A similar approach can be used to create optical elements for the SPP. Attention is given to the mechanisms of phase modulation of the SPP and plasmonic modes of thin metal films using dielectric ridges of variable height and fixed length (or variable height and length), located on the surface of the metal. The section also describes the method of suppressing parasitic scattering in diffraction of SPP (plasmonic modes) on a dielectric ridge, based on the use of the structure of two isotropic dielectric layers on the metal surface. This two-layer structure enables phase modulation of the SPP (plasmonic modes) while reducing the energy loss in the parasitic scattering by an order of magnitude compared to single-layer steps. The section also presents the results of calculation and research of diffractive lenses for focusing the SPP based on different phase modulation methods.

The main practical application of the results of this section is the creation of high-performance elements of plasmon optics: lens, Bragg gratings, plasmonic crystals.

In section 1.5 the Fourier modal method is applied to the problem of calculating diffraction gratings which generate interference patterns of evanescent electromagnetic waves in the near field (especially of plasmonic modes). The first part of the section provides a theoretical description of the interference patterns of evanescent diffraction orders for gratings with one- and two-dimensional periodicity. This is followed by a series of numerical examples of the gratings lattices, forming interference patterns of evanescent electromagnetic waves and plasmonic modes with a substantially subwavelength period, and the ability to control the type and period of the interference patterns due to changes in the parameters of the incident radiation is demonstrated.

The practical use of the results of this section includes contact nanolithography systems and systems for optical trapping and manipulation of nanoscale objects.

\subsection{The Fourier modal method of solving the diffraction problems}

\subsubsection{The equation of a plane wave}

This subsection is auxiliary. It considers the derivation of the general equation of a plane wave in an isotropic medium used in the following description of the Fourier modal method. 
We write the Maxwell equations and material equations in the Gaussian system of units:

$$
\left\{\begin{array}{l}
\nabla \times \mathbf{E}=-\frac{1}{c} \frac{\partial}{\partial t} \mathbf{B}, \\
\nabla \times \mathbf{H}=\frac{1}{c} \frac{\partial}{\partial t} \mathbf{D}, \\
\mathbf{B}=\mu \mathbf{H}, \\
\mathbf{D}=\varepsilon \mathbf{E} .
\end{array}\right.
$$

Equations (1.1) are written in the absence of charges, in this case $\varepsilon$ is the complex permittivity. For a monochromatic field

$$
\begin{aligned}
& \mathbf{E}(x, y, z, t)=\mathbf{E}(x, y, z) \exp (-\mathrm{i} \omega t), \\
& \mathbf{H}(x, y, z, t)=\mathbf{H}(x, y, z) \exp (-\mathrm{i} \omega t),
\end{aligned}
$$

system (1.1) takes the form:

$$
\left\{\begin{array}{l}
\nabla \times \mathbf{E}=\mathrm{i} k_{0} \mu \mathbf{H}, \\
\nabla \times \mathbf{H}=-\mathrm{i} k_{0} \varepsilon \mathbf{E},
\end{array}\right.
$$

wherein $k_{0}=2 \pi / \lambda, \lambda$ is the wavelength. Expanding the rotor operator we get

$$
\nabla \times \mathbf{E}=\left[\begin{array}{l}
\frac{\partial E_{z}}{\partial y}-\frac{\partial E_{y}}{\partial z} \\
\frac{\partial E_{x}}{\partial z}-\frac{\partial E_{z}}{\partial x} \\
\frac{\partial E_{y}}{\partial x}-\frac{\partial E_{x}}{\partial y}
\end{array}\right]=\mathrm{i} k_{0} \mu\left[\begin{array}{c}
H_{x} \\
H_{y} \\
H_{z}
\end{array}\right], \nabla \times \mathbf{H}=\left[\begin{array}{c}
\frac{\partial H_{z}}{\partial y}-\frac{\partial H_{y}}{\partial z} \\
\frac{\partial H_{x}}{\partial z}-\frac{\partial H_{z}}{\partial x} \\
\frac{\partial H_{y}}{\partial x}-\frac{\partial H_{x}}{\partial y}
\end{array}\right]=-\mathrm{i} k_{0} \varepsilon\left[\begin{array}{c}
E_{x} \\
E_{y} \\
E_{z}
\end{array}\right] .
$$

To obtain the plane wave equation we will seek the solution of (1.4) in the form

$$
\Phi(x, y, z)=\Phi \exp \left(\mathrm{i} k_{0}(\alpha x+\beta y+\gamma z)\right)
$$

where $\Phi=\left[\begin{array}{llllll}E_{x} & E_{y} & E_{z} & H_{x} & H_{y} & H_{z}\end{array}\right]^{\mathrm{T}}$ is the column vector of the components of the field. The values $\alpha, \beta, \gamma$ in (1.5) define the direction of propagation of the plane wave. Substituting (1.5) into 
(1.4), we obtain

$\mathrm{i} k_{0}\left[\begin{array}{c}\beta E_{z}-\gamma E_{y} \\ \gamma E_{x}-\alpha E_{z} \\ \alpha E_{y}-\beta E_{x}\end{array}\right]=\mathrm{i} k_{0} \mu\left[\begin{array}{c}H_{x} \\ H_{y} \\ H_{z}\end{array}\right], \quad \mathrm{i} k_{0}\left[\begin{array}{c}\beta H_{z}-\gamma H_{y} \\ \gamma H_{x}-\alpha H_{z} \\ \alpha H_{y}-\beta H_{x}\end{array}\right]=-\mathrm{i} k_{0} \varepsilon\left[\begin{array}{c}E_{x} \\ E_{y} \\ E_{z}\end{array}\right]$.

We represent the equation (1.6) in the matrix form:

$$
\left[\begin{array}{cccccc}
0 & -\gamma & \beta & -\mu & 0 & 0 \\
\gamma & 0 & -\alpha & 0 & -\mu & 0 \\
-\beta & \alpha & 0 & 0 & 0 & -\mu \\
\varepsilon & 0 & 0 & 0 & -\gamma & \beta \\
0 & \varepsilon & 0 & \gamma & 0 & -\alpha \\
0 & 0 & \varepsilon & -\beta & \alpha & 0
\end{array}\right] \boldsymbol{\Phi}=\mathbf{A} \cdot \boldsymbol{\Phi}=\mathbf{0}
$$

Direct calculation shows that the determinant of the system (1.7) has the form:

$$
\operatorname{det} \mathbf{A}=\varepsilon \mu\left(\alpha^{2}+\beta^{2}+\gamma^{2}-\varepsilon \mu\right)^{2}
$$

The sought non-trivial solution of the system (1.7) exists when the determinant (1.8) is zero. If $\mu=1$ the determinant of (1.8) vanishes provided

$$
\alpha^{2}+\beta^{2}+\gamma^{2}=\varepsilon
$$

We write down explicitly the solution of (1.6). Under the condition (1.9) the rank of the system (1.7) is equal to four, so to write the solution the values of the amplitudes $E_{z}$ and $H_{z}$ are fixed. We introduce the so-called E- and H-waves. For the E-wave $E_{z} \neq 0$, $H_{z}=0$, and for the H-wave $H_{z} \neq 0, E_{z}=0$. We represent the desired solution as a superposition of the E- and $\mathrm{H}$-waves in the form

$$
\Phi=\frac{1}{\sqrt{\alpha^{2}+\beta^{2}}}\left[\begin{array}{c}
-\alpha \gamma \\
-\beta \gamma \\
\alpha^{2}+\beta^{2} \\
\beta \varepsilon \\
-\alpha \varepsilon \\
0
\end{array}\right] \frac{A_{E}}{\sqrt{\varepsilon}}+\frac{1}{\sqrt{\alpha^{2}+\beta^{2}}}\left[\begin{array}{c}
-\beta \\
\alpha \\
0 \\
-\alpha \gamma \\
-\beta \gamma \\
\alpha^{2}+\beta^{2}
\end{array}\right] A_{H}
$$


where $A_{E}$ and $A_{H}$ are the the amplitudes of the E- and H-waves, respectively.

We write the components of the vector $\mathbf{p}=(\alpha, \beta, \gamma)$, representing the propagation direction of the wave, by means of the angles $\theta$ and $\varphi$ of the spherical coordinate system:

$$
\alpha=\sqrt{\varepsilon} \cos \varphi \sin \theta, \beta=\sqrt{\varepsilon} \sin \varphi \sin \theta, \gamma=\sqrt{\varepsilon} \cos \theta,
$$

where $\theta$ is the angle between the vector $\mathbf{p}$ and the axis $O z, \varphi$ is the angle between the plane of incidence and the plane $x \mathrm{Oz}$. For these angles the following relations are fulfilled:

$$
\sqrt{\alpha^{2}+\beta^{2}}=\sqrt{\varepsilon} \sin \theta, \frac{\alpha}{\sqrt{\alpha^{2}+\beta^{2}}}=\cos \varphi, \frac{\beta}{\sqrt{\alpha^{2}+\beta^{2}}}=\sin \varphi .
$$

We write separately $y$ - and $x$-components of the electric and magnetic fields:

$$
\begin{aligned}
\bar{\Phi} & =\left[\begin{array}{l}
E_{y} \\
E_{x} \\
H_{y} \\
H_{x}
\end{array}\right]=\left[\begin{array}{c}
-\cos \theta \sin \varphi \\
-\cos \theta \cos \varphi \\
-\sqrt{\varepsilon} \cos \varphi \\
\sqrt{\varepsilon} \sin \varphi
\end{array}\right] A_{E}+\left[\begin{array}{c}
\cos \varphi \\
-\sin \varphi \\
-\sqrt{\varepsilon} \cos \theta \sin \varphi \\
-\sqrt{\varepsilon} \cos \theta \cos \varphi
\end{array}\right] A_{H} \\
& =\left[\begin{array}{ll}
\mathbf{R} & 0 \\
0 & \mathbf{R}
\end{array}\right]\left[\begin{array}{cc}
0 & 1 \\
-\gamma / \sqrt{\varepsilon} & 0 \\
-\sqrt{\varepsilon} & 0 \\
0 & -\gamma
\end{array}\right]\left[\begin{array}{l}
A_{E} \\
A_{H}
\end{array}\right],
\end{aligned}
$$

where $\mathbf{R}$ is the rotation matrix of the following form:

$$
\mathbf{R}=\left[\begin{array}{cc}
\cos \varphi & \sin \varphi \\
-\sin \varphi & \cos \varphi
\end{array}\right] .
$$

To describe the polarization of the wave, we introduce the angle $\psi-$ the angle between the vector $\mathbf{E}$ and the incidence plane. The incidence plane is the plane containing the direction of the wave propagation vector $\mathbf{p}$ and the axis $\mathrm{Oz}$. In this case

$$
\begin{aligned}
& A_{H}=A \sin \psi, \\
& A_{E}=A \cos \psi .
\end{aligned}
$$


Note that in the solution of the diffraction problem the case $\varphi$ $=0$ corresponds to the so-called planar diffraction. In this case the E-waves are waves with TM-polarization and $\mathrm{H}$-waves the waves with TE-polarization.

Consider the time-averaged Umov-Poynting vector $\mathbf{S}=$ $1 / 2 \operatorname{Re}\left(\mathbf{E} \times \mathbf{H}^{*}\right)$. We compute the $z$-component of the vector, which corresponds to the density of the energy flux through the plane $\mathrm{xOy}$. For the E-wave

$$
2 S_{z}=\operatorname{Re}\left(E_{x} H_{y}^{*}-E_{y} H_{x}^{*}\right)=\left|A_{E}\right|^{2} \operatorname{Re}\left(\sqrt{\varepsilon}^{*}\right) \cdot \cos \theta=\left|A_{E}\right|^{2} \operatorname{Re} \sqrt{\varepsilon} \cdot \cos \theta,
$$

and similarly for the H-wave:

$$
2 S_{z}=\operatorname{Re}\left(E_{x} H_{y}^{*}-E_{y} H_{x}^{*}\right)=\left|A_{H}\right|^{2} \operatorname{Re}\left(\sqrt{\varepsilon}^{*}\right) \cdot \cos \theta=\left|A_{H}\right|^{2} \operatorname{Re} \sqrt{\varepsilon} \cdot \cos \theta,
$$

The expressions (1.16) and (1.17) describe the energy flux through the plane $x O y$. These expressions are important for the control of the energy conservation law in solving the problem of diffraction on diffraction structures of a lossless material.

\subsubsection{The Fourier modal method for two-dimensional periodic structures}

The Fourier modal method focuses on the numerical solution of Maxwell's equations for the case of periodic structures, consisting of a set of 'binary layers' [1-13]. In each layer, dielectric permittivity (magnetic permeability) of the material of the structure is independent of the variable $z$ and the axis $O z$ is perpendicular to the structure. In the method the electromagnetic field in the areas above and below the structure is given as a superposition of plane waves (diffraction orders). The function of dielectric permittivity and magnetic permeability of the material in each layer of the structure are represented as segments of the Fourier series, and the components of the electromagnetic field are written as an expansion in the Fourier modes basis. The calculation of the Fourier modes is reduced to eigenvalue problems. Sequential imposition of the condition of equality of the tangential components of the electromagnetic field at the boundaries of the layers reduces the determination of the amplitudes of the reflected and transmitted diffraction orders to solving a system of linear equations [1-13]. 
Here the method is considered for the two-dimensional diffraction gratings (gratings with one-dimensional periodicity). In the two-dimensional case, the material properties of the structure are constant along one of the axes. The structure materials are in a general case defined by tensors of permittivity and permeability.

\subsubsection{The geometry of the structure and formulation of the problem}

Consider a diffraction grating with a period $d$ along the axis $x$ (Fig. 1.1). For gratings with a continuous profile (dashed line in Fig. 1.1) the method approximates the grating profile by a set of binary layers. We assume that the diffraction grating is made up of $L$ binary layers (Fig. 1.1). The permittivity $\varepsilon$ and permeability $\mu$ in the layers depend only on the variable $x$. Layer boundaries are the lines $z=d_{i}$, the $l$-th layer is located in the area $z_{l}<z<z_{l-1}$.

Over the considered structure in the region $z>z_{0}=0$ there is a homogeneous dielectric with a refractive index $n_{\mathrm{U}}=\sqrt{\varepsilon_{\mathrm{U}}}$. Under the structure there is a homogeneous dielectric with a refractive index $n_{\mathrm{D}}=\sqrt{\varepsilon_{\mathrm{D}}}$.

A plane monochromatic wave is incident on the top of the grating (wavelength $\lambda$, wave number $k_{0}=\frac{2 \pi}{\lambda}$ ), the direction of which is defined by the angles $\theta$ and $\varphi$ in the spherical coordinate system

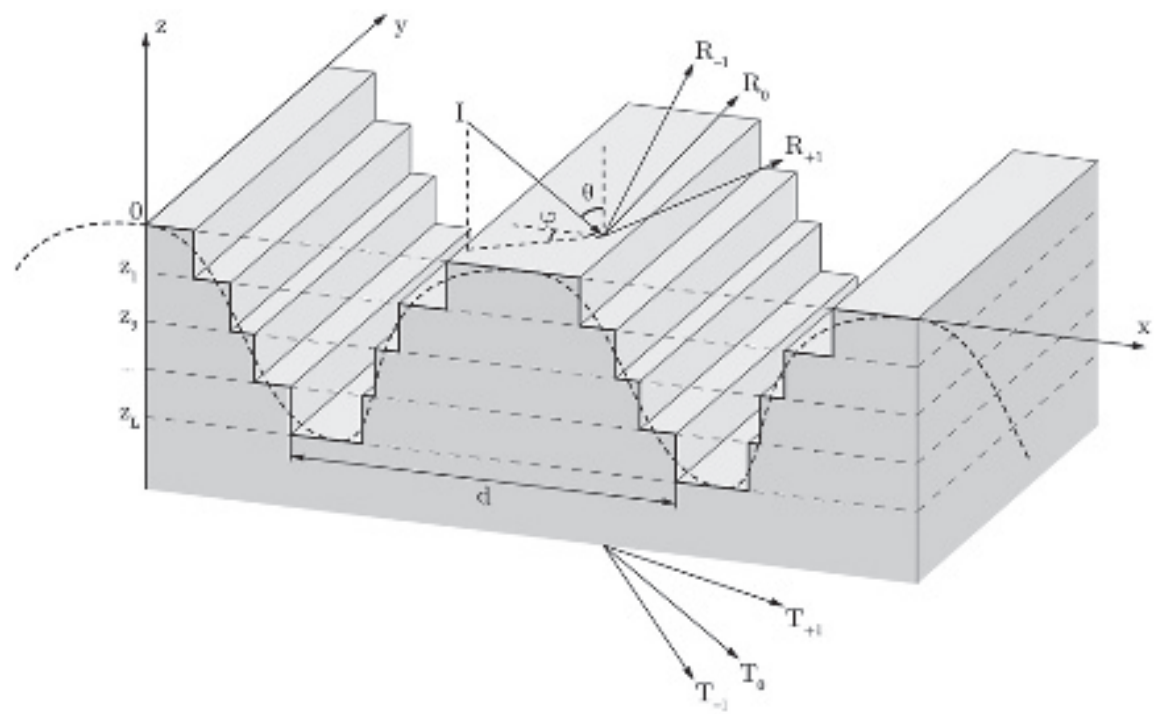

Fig. 1.1. The geeometry of the problem. 
(Fig. 1.1). The polarization of the wave is determined by the angle $\psi$ between the incidence plane and the vector $\mathbf{E}$ (see. (1.15)).

The problem of light diffraction by a periodic structure is solved by calculating the intensities or complex amplitudes of diffraction orders. The diffraction orders are the reflected and transmitted plane waves arising in the diffraction of the incident wave on the structure. There are reflected orders with amplitudes $R_{i}, i=0, \pm 1$, $\pm 2, \ldots$ and transmitted orders with amplitudes $T_{i}, i=0, \pm 1, \pm 2, \ldots$ (Fig. 1.1). The diffraction orders can also be divided into evanescent and propagating. The amplitude of the evanescent orders decreases exponentially away from the gratings.

\subsubsection{Description of the field above and below the structure}

The field above and below the structure is written as a superposition of plane waves (Rayleigh expansion). According to (1.5), (1.10), the plane wave is represented by a vector of six components:

$$
\Phi=\left[\begin{array}{llllll}
E_{x} & E_{y} & E_{z} & H_{x} & H_{y} & H_{z}
\end{array}\right]^{T} .
$$

The equation of the incident wave can be represented in the form

$$
\Phi^{\mathrm{inc}}(x, y, z)=\Phi^{\mathrm{inc}}(\psi) \exp \left(i\left(k_{x, 0} x+k_{y} y-k_{z, U, 0} z\right)\right),
$$

where $\Phi^{\text {inc }}(\psi)$ denotes the dependence of the incident wave on polarization (see (1.15)). The constants $k_{x, 0}=k_{0} n_{U} \sin \theta \cos \varphi$, $k_{x, 0}=k_{0} n_{\mathrm{U}} \sin \theta \cos \varphi, \quad k_{y}=k_{0} n_{\mathrm{U}} \sin \theta \sin \varphi, \quad k_{z, \mathrm{U}, 0}=\sqrt{\left(k_{0} n_{\mathrm{U}}\right)^{2}-k_{x, 0}^{2}-k_{y}^{2}}$ in (1.19) are determined through the angles $\theta$ and $\varphi$ defining the direction of the incident wave.

The field above the grating corresponds to the superposition of the incident wave and the reflected diffraction orders:

$$
\Phi^{\mathrm{U}}(x, y, z)=\Phi^{\mathrm{inc}}(x, y, z)+\sum_{m} \Phi_{m}^{\mathrm{R}}\left(R_{m}\right) \exp \left(i\left(k_{x, m} x+k_{y} y+k_{z, \mathrm{U}, m} z\right)\right),
$$

where $\boldsymbol{\Phi}(R)=\boldsymbol{\Phi}_{E} \mathrm{R}_{E}+\boldsymbol{\Phi}_{H} \mathrm{R}_{H}$. Expression $\boldsymbol{\Phi}(R)$ corresponds to the representation (1.10) of the reflected wave as a sum of E- and H-waves with complex amplitudes $R_{E}$ and $R_{H}$ respectively.

The field below the grating is expressed similarly as a superposition of transmitted waves:

$$
\Phi^{\mathrm{D}}(x, y, z)=\sum_{m} \Phi_{m}^{T}\left(T_{m}\right) \exp \left(i\left(k_{x, m} x+k_{y} y-k_{z, \mathrm{D}, m}\left(z-z_{L}\right)\right)\right) .
$$


The directions of the orders in (1.20) (1.21) are given by propagation constants $k_{x, m}, k_{y}, k_{z, p, m}$. The propagation constants have the form

$$
\begin{aligned}
& k_{x, m}=k_{0}\left(n_{\mathrm{U}} \sin \theta \cos \varphi+m \frac{\lambda}{d}\right), \\
& k_{y}=k_{0} n_{\mathrm{U}} \sin \theta \sin \varphi, \\
& k_{z, p, m}=\sqrt{\left(k_{0} n_{p}\right)^{2}-k_{x, m}^{2}-k_{y}^{2}},
\end{aligned}
$$

where $p$ is taken as ' $\mathrm{U}$ ' for the reflected orders (the field above the grid) and ' $\mathrm{D}$ ' - for the transmitted orders (the field under the grating). The expression for $k_{x, m}$ follows from the Floquet-Bloch theorem $[14,15]$. From a mathematical point of view the form of $k_{x, m}$ provides the implementation of the so-called quasi-periodicity condition

$$
\Phi^{p}(x+d, y, z)=\Phi^{p}(x, y, z) \exp \left(i k_{x, 0} d\right), p=\mathrm{U}, \mathrm{D} .
$$

According to (1.23), the amplitude of the field does not change with shifts by a period. The waves with real $k_{z, p, m}$ are propagating, those with imaginary one - evanescent.

1.1.2.3. The system of differential equations for the description of the field in the layer

We turn now to the field within the particular $l$-th layer. For simplicity, we omit the index $l$.

The field in each layer is described by the Maxwell's equations for the monochromatic field in the form

$$
\left\{\begin{array}{l}
\nabla \times \mathbf{E}=i k_{0} \overrightarrow{\boldsymbol{\mu}} \mathbf{H}, \\
\nabla \times \mathbf{H}=-i k_{0} \overrightarrow{\mathbf{\varepsilon}} \mathbf{E},
\end{array}\right.
$$

where $\overrightarrow{\boldsymbol{\varepsilon}}, \overrightarrow{\boldsymbol{\mu}}$ are tensors in the most general case:

$$
\overrightarrow{\boldsymbol{\varepsilon}}=\left[\begin{array}{lll}
\varepsilon_{1,1} & \varepsilon_{1,2} & \varepsilon_{1,3} \\
\varepsilon_{2,1} & \varepsilon_{2,2} & \varepsilon_{2,3} \\
\varepsilon_{3,1} & \varepsilon_{3,2} & \varepsilon_{3,3}
\end{array}\right], \quad \overrightarrow{\boldsymbol{\mu}}=\left[\begin{array}{lll}
\mu_{1,1} & \mu_{1,2} & \mu_{1,3} \\
\mu_{2,1} & \mu_{2,2} & \mu_{2,3} \\
\mu_{3,1} & \mu_{3,2} & \mu_{3,3}
\end{array}\right] .
$$


The tensor components depend only on the coordinate $x$. Expanding the rotor operator (1.24), we obtain:

$$
\begin{cases}\frac{\partial E_{z}}{\partial y}-\frac{\partial E_{y}}{\partial z} & =i k_{0}\left(\mu_{1,1} H_{x}+\mu_{1,2} H_{y}+\mu_{1,3} H_{z}\right), \\ \frac{\partial E_{x}}{\partial z}-\frac{\partial E_{z}}{\partial x} & =i k_{0}\left(\mu_{2,1} H_{x}+\mu_{2,2} H_{y}+\mu_{2,3} H_{z}\right), \\ \frac{\partial E_{y}}{\partial x}-\frac{\partial E_{x}}{\partial y} & =i k_{0}\left(\mu_{3,1} H_{x}+\mu_{3,2} H_{y}+\mu_{3,3} H_{z}\right), \\ \frac{\partial H_{z}}{\partial y}-\frac{\partial H_{y}}{\partial z} & =-i k_{0}\left(\varepsilon_{1,1} E_{x}+\varepsilon_{1,2} E_{y}+\varepsilon_{1,3} E_{z}\right), \\ \frac{\partial H_{x}}{\partial z}-\frac{\partial H_{z}}{\partial x} & =-i k_{0}\left(\varepsilon_{2,1} E_{x}+\varepsilon_{2,2} E_{y}+\varepsilon_{2,3} E_{z}\right), \\ \frac{\partial H_{y}}{\partial x}-\frac{\partial H_{x}}{\partial y} & =-i k_{0}\left(\varepsilon_{3,1} E_{x}+\varepsilon_{3,2} E_{y}+\varepsilon_{3,3} E_{z}\right) .\end{cases}
$$

We represent the components of the electric and magnetic fields in the form of Fourier series with respect to variable $x$ :

$$
\left\{\begin{array}{l}
E_{x}=\sum_{j} S_{x, j}(z) \exp \left(i\left(k_{x, j} x+k_{y} y\right)\right), \\
E_{y}=\sum_{j} S_{y, j}(z) \exp \left(i\left(k_{x, j} x+k_{y} y\right)\right), \\
E_{z}=\sum_{j} S_{z, j}(z) \exp \left(i\left(k_{x, j} x+k_{y} y\right)\right), \\
H_{x}=-i \sum_{j} U_{x, j}(z) \exp \left(i\left(k_{x, j} x+k_{y} y\right)\right), \\
H_{y}=-i \sum_{j} U_{y, j}(z) \exp \left(i\left(k_{x, j} x+k_{y} y\right)\right), \\
H_{z}=-i \sum_{j} U_{z, j}(z) \exp \left(i\left(k_{x, j} x+k_{y} y\right)\right) .
\end{array}\right.
$$

The representations (1.27) are written with the quasi-periodicity of the field components with respect to variable $x$ taken into account. We restrict ourselves to a finite number of terms in the expansion (1.27), corresponding to $-N \leq j \leq N$. From variables $S_{x, j}, S_{y, j}, S_{z, j}, U_{x, j}$, $U_{y, j}, U_{z, j}$, we form column vectors, $\boldsymbol{S}_{x}, \boldsymbol{S}_{y}, \boldsymbol{S}_{z}, \boldsymbol{U}_{x}, \boldsymbol{U}_{y}, \boldsymbol{U}_{z}$, containing $2 N+1$ elements each. 
Consider the expansion of the product of two functions $\varepsilon_{a}(x) E_{b}(x, y, z)$ into the Fourier series:

$$
\begin{aligned}
\varepsilon_{a}(x) E_{b}(x) & =\left(\sum_{m} e_{a, m} \exp \left(i \frac{2 \pi}{d} m x\right)\right) \cdot\left(\sum_{l} S_{b, l}(z) \exp \left(i\left(k_{x, l} x+k_{y} y\right)\right)\right) \\
& =[j=l+m]=\sum_{j}\left(\sum_{l} e_{a, j-l} S_{b, l}(z)\right) \exp \left(i\left(k_{x, j} x+k_{y} y\right)\right) .
\end{aligned}
$$

Retaining a finite number of terms corresponding to $-N \leq j \leq N,-N \leq l \leq N$ in (1.28), we obtain:

$$
\left[\varepsilon_{a}(x) E_{b}(x)\right]=\llbracket \varepsilon_{a}(x) \rrbracket \mathbf{S}_{b}=\llbracket \varepsilon_{a}(x) \rrbracket\left[E_{b}(x)\right],
$$

where the square brackets denote the vectors composed from the Fourier coefficients of the expansion of functions $\varepsilon_{a}(x) E_{b}(x)$ and $E_{b}(x)$, and $\llbracket \varepsilon_{a}(x) \rrbracket$ is the Toeplitz matrix of the Fourier coefficients, which has the following form:

$$
\mathbf{Q}=\left[\begin{array}{cccccc}
e_{0} & e_{-1} & e_{-2} & \cdots & \cdots & e_{-2 N} \\
e_{1} & e_{0} & e_{-1} & e_{-2} & \ddots & e_{1-2 N} \\
e_{2} & e_{1} & e_{0} & e_{-1} & \ddots & \vdots \\
\vdots & e_{2} & e_{1} & \ddots & \ddots & \vdots \\
\vdots & \ddots & \ddots & \ddots & \ddots & e_{-1} \\
e_{2 N} & e_{2 N-1} & \cdots & \cdots & e_{1} & e_{0}
\end{array}\right] .
$$

Substituting (1.27) into (1.26) and equating coefficients of the same Fourier harmonics, we obtain:

$$
\begin{cases}i k_{0} \mathbf{K}_{y} \mathbf{S}_{z}-\frac{d \mathbf{S}_{y}}{d z} & =k_{0}\left(\mathbf{M}_{1,1} \mathbf{U}_{x}+\mathbf{M}_{1,2} \mathbf{U}_{y}+\mathbf{M}_{1,3} \mathbf{U}_{z}\right), \\ \frac{d \mathbf{S}_{x}}{d z}-i k_{0} \mathbf{K}_{x} \mathbf{S}_{z} & =k_{0}\left(\mathbf{M}_{2,1} \mathbf{U}_{x}+\mathbf{M}_{2,2} \mathbf{U}_{y}+\mathbf{M}_{2,3} \mathbf{U}_{z}\right) \\ i k_{0} \mathbf{K}_{x} \mathbf{S}_{y}-i k_{0} \mathbf{K}_{y} \mathbf{S}_{x} & =k_{0}\left(\mathbf{M}_{3,1} \mathbf{U}_{x}+\mathbf{M}_{3,2} \mathbf{U}_{y}+\mathbf{M}_{3,3} \mathbf{U}_{z}\right), \\ i k_{0} \mathbf{K}_{y} \mathbf{U}_{z}-\frac{d \mathbf{U}_{y}}{d z} & =k_{0}\left(\mathbf{E}_{1,1} \mathbf{S}_{x}+\mathbf{E}_{1,2} \mathbf{S}_{y}+\mathbf{E}_{1,3} \mathbf{S}_{z}\right) \\ \frac{d \mathbf{U}_{x}}{d z}-i k_{0} \mathbf{K}_{x} \mathbf{U}_{z} & =k_{0}\left(\mathbf{E}_{2,1} \mathbf{S}_{x}+\mathbf{E}_{2,2} \mathbf{S}_{y}+\mathbf{E}_{2,3} \mathbf{S}_{z}\right) \\ i k_{0} \mathbf{K}_{x} \mathbf{U}_{y}-i k_{0} \mathbf{K}_{y} \mathbf{U}_{x} & =k_{0}\left(\mathbf{E}_{3,1} \mathbf{S}_{x}+\mathbf{E}_{3,2} \mathbf{S}_{y}+\mathbf{E}_{3,3} \mathbf{S}_{z}\right),\end{cases}
$$


where $\mathbf{K}_{x}=\underset{j}{\operatorname{diag}} \frac{k_{x, j}}{k_{0}}, \mathbf{K}_{y}=\operatorname{diag} \frac{k_{y}}{k_{0}}-$ diagonal matrices, $\mathbf{E}_{i, j}=\llbracket \varepsilon_{i, j} \rrbracket$ and $\mathbf{M}_{i, j}=\llbracket \mu_{i, j} \rrbracket$ are the Toeplitz matrices of the form (1.30), made up of the expansion coefficients in the Fourier series $\varepsilon_{i, j}(x)$ and $\mu_{i, j}(x)$.

We perform a change of variables $z^{\prime}=k_{0} z$ and transform the system (1.31) to the form:

$$
\left\{\begin{array}{cl}
-\frac{d \mathbf{S}_{y}}{d z^{\prime}} & =\mathbf{M}_{1,1} \mathbf{U}_{x}+\mathbf{M}_{1,2} \mathbf{U}_{y}+\mathbf{M}_{1,3} \mathbf{U}_{z}-i \mathbf{K}_{y} \mathbf{S}_{z}, \\
-\frac{d \mathbf{S}_{x}}{d z^{\prime}} & =-\mathbf{M}_{2,1} \mathbf{U}_{x}-\mathbf{M}_{2,2} \mathbf{U}_{y}-\mathbf{M}_{2,3} \mathbf{U}_{z}-i \mathbf{K}_{x} \mathbf{S}_{z}, \\
-\frac{d \mathbf{U}_{y}}{d z^{\prime}} & =\mathbf{E}_{1,1} \mathbf{S}_{x}+\mathbf{E}_{1,2} \mathbf{S}_{y}+\mathbf{E}_{1,3} \mathbf{S}_{z}-i \mathbf{K}_{y} \mathbf{U}_{z} \\
-\frac{d \mathbf{U}_{x}}{d z^{\prime}} & =-\mathbf{E}_{2,1} \mathbf{S}_{x}-\mathbf{E}_{2,2} \mathbf{S}_{y}-\mathbf{E}_{2,3} \mathbf{S}_{z}-i \mathbf{K}_{x} \mathbf{U}_{z} \\
\mathbf{E}_{3,3} \mathbf{S}_{z} & =i \mathbf{K}_{x} \mathbf{U}_{y}-i \mathbf{K}_{y} \mathbf{U}_{x}-\mathbf{E}_{3,1} \mathbf{S}_{x}-\mathbf{E}_{3,2} \mathbf{S}_{y} \\
\mathbf{M}_{3,3} \mathbf{U}_{z} & =i \mathbf{K}_{x} \mathbf{S}_{y}-i \mathbf{K}_{y} \mathbf{S}_{x}-\mathbf{M}_{3,1} \mathbf{U}_{x}-\mathbf{M}_{3,2} \mathbf{U}_{y}
\end{array}\right.
$$

We rewrite the first four equations of the system in matrix form:

$$
\frac{d}{d z^{\prime}}\left[\begin{array}{l}
\mathbf{S}_{y} \\
\mathbf{S}_{x} \\
\mathbf{U}_{y} \\
\mathbf{U}_{x}
\end{array}\right]=\mathbf{Y}\left[\begin{array}{l}
\mathbf{S}_{y} \\
\mathbf{S}_{x} \\
\mathbf{U}_{y} \\
\mathbf{U}_{x}
\end{array}\right]-\left[\begin{array}{cc}
-i \mathbf{K}_{y} & \mathbf{M}_{1,3} \\
-i \mathbf{K}_{x} & -\mathbf{M}_{2,3} \\
\mathbf{E}_{1,3} & -i \mathbf{K}_{y} \\
-\mathbf{E}_{2,3} & -i \mathbf{K}_{x}
\end{array}\right]\left[\begin{array}{l}
\mathbf{S}_{z} \\
\mathbf{U}_{z}
\end{array}\right],
$$

where

$$
\mathbf{Y}=-\left[\begin{array}{cccc}
\mathbf{0} & \mathbf{0} & \mathbf{M}_{1,2} & \mathbf{M}_{1,1} \\
\mathbf{0} & \mathbf{0} & -\mathbf{M}_{2,2} & -\mathbf{M}_{2,1} \\
\mathbf{E}_{1,2} & \mathbf{E}_{1,1} & \mathbf{0} & \mathbf{0} \\
-\mathbf{E}_{2,2} & -\mathbf{E}_{2,1} & \mathbf{0} & \mathbf{0}
\end{array}\right]
$$

From the last two equations of (1.32) we express $S_{z}$ and $U_{z}$ and substitute the resulting expressions into (1.33). As a result, we obtain 
a system of linear differential equations with respect to $x$ - and $y$ Fourier components of the fields:

where

$$
\frac{d}{d z^{\prime}}\left[\begin{array}{l}
\mathbf{S}_{y} \\
\mathbf{S}_{x} \\
\mathbf{U}_{y} \\
\mathbf{U}_{x}
\end{array}\right]=\mathbf{A}\left[\begin{array}{c}
\mathbf{S}_{y} \\
\mathbf{S}_{x} \\
\mathbf{U}_{y} \\
\mathbf{U}_{x}
\end{array}\right]
$$

where

$$
\mathbf{A}=\mathbf{Y}-\left[\begin{array}{cc}
i \mathbf{K}_{y} & -\mathbf{M}_{1,3} \\
i \mathbf{K}_{x} & \mathbf{M}_{2,3} \\
-\mathbf{E}_{1,3} & i \mathbf{K}_{y} \\
\mathbf{E}_{2,3} & i \mathbf{K}_{x}
\end{array}\right]\left[\begin{array}{cc}
\mathbf{E}_{3,3}^{-1} & \mathbf{0} \\
\mathbf{0} & \mathbf{M}_{3,3}^{-1}
\end{array}\right]\left[\begin{array}{cccc}
\mathbf{E}_{3,2} & \mathbf{E}_{3,1} & -i \mathbf{K}_{x} & i \mathbf{K}_{y} \\
-i \mathbf{K}_{x} & i \mathbf{K}_{y} & \mathbf{M}_{3,2} & \mathbf{M}_{3,1}
\end{array}\right]
$$

Thus, we have obtained a system of linear differential equations of the first order for the vectors $\mathbf{S}_{x}, \mathbf{S}_{y}, \mathbf{U}_{x}, \mathbf{U}_{y}$. Note that the matrix A has the dimensions $4(2 N+1) \times 4(2 N+1)$.

\section{Correct rules of the Fourier expansion of the product of functions}

The derivation of the system of differential equations (1.35) was based on the representation in (1.26) of the component of electromagnetic fields and of the components of permittivity and permeability tensors in the form of segments of the Fourier series. The representation of the products of the functions in (1.26) by the Fourier series has its own peculiarities. The used formulas (1.28)(1.30) have limited applicavility.

Consider two periodic functions

$$
f(x)=\sum_{m} f_{m} \exp (i K m x), g(x)=\sum_{m} g_{m} \exp (i K m x), K=\frac{2 \pi}{d}
$$

and the expansion of their product into the Fourier series 


$$
h(x)=f(x) g(x)=\sum_{m} h_{m} \exp (i K m x) .
$$

The Fourier coefficients of the product are the values

$$
h_{j}=\sum_{m=-N}^{N} f_{j-m} g_{m}
$$

obtained by direct multiplication of the series (1.37), (1.38). Equation (1.39) for calculating the Fourier coefficients is called the Laurent rule. In matrix notation (1.39) can be represented as [5]

$$
[h]=\llbracket f \rrbracket[g],
$$

where, as in (1.29), the square brackets denote the vectors composed from the Fourier coefficients of the expansion of the functions, and $\llbracket f \rrbracket$ is the Toeplitz matrix of the Fourier coefficients.

As was shown in $[5,6]$, the use of formula $(1.40)$ is correct if there is no value $x$ for which the functions $f(x)$ and $g(x)$ show a discontinuity at the same time. Using the Laurent rule (1.40) for the product of the functions having identical points of discontinuity leads to poor convergence of the Fourier series at the points of discontinuity.

If the functions $f(x)$ and $g(x)$ are discontinuous at the same time, but the function $h(x)=f(x) g(x)$ is continuous, it is correct to use the so-called inverse Laurent rules [5]:

$$
[h]=\llbracket \frac{1}{f} \rrbracket^{-1}[g] .
$$

In the products $\mu_{i, 1}(x) \cdot \mathrm{H}_{x}(x, y, z)$ and $\varepsilon_{i, 1}(x) \cdot \mathrm{E}_{x}(x, y, z)$ in (1.26), both expanded functions have discontinuities at the same points $x$, corresponding to vertical interfaces of the media in the layers. Accordingly, it is erroneous to use the Laurent rule (1.28)-(1.30) when writing (1.31). Errors in the use of the Laurent rule are significant when working with diffraction gratings of conductive materials. In particular, the erroneous use of the Laurent rule leads to slow convergence of the solutions for binary metal gratings in the case of TM-polarization [5-10]. In this case we define the convergence as the stabilization of the results of the calculation of the amplitudes of diffraction orders with increasing number of the Fourier harmonics $N$ in the components of the field (1.27). 
Continuous components at the vertical boundaries between the media are the tangential components $E_{y}, E_{z}, H_{y}, H_{z}$ and the normal components of the electric displacement and magnetic induction $D_{x}, B_{x}$. We express discontinuous components $E_{x}, H_{x}$ through the continuous field components

$$
\begin{aligned}
& E_{x}=\frac{1}{\varepsilon_{11}} D_{x}-\frac{\varepsilon_{12}}{\varepsilon_{11}} E_{y}-\frac{\varepsilon_{13}}{\varepsilon_{11}} E_{z}, \\
& H_{x}=\frac{1}{\mu_{11}} B_{x}-\frac{\mu_{12}}{\mu_{11}} H_{y}-\frac{\mu_{13}}{\mu_{11}} H_{z}
\end{aligned}
$$

and substitute into Maxwell's equations (1.26). The result is:

$$
\begin{aligned}
& \int \frac{\partial E_{z}}{\partial y}-\frac{\partial E_{y}}{\partial z}=i k_{0} B_{x}, \\
& \frac{\partial E_{x}}{\partial z}-\frac{\partial E_{z}}{\partial x}=i k_{0}\left(\frac{\propto_{2,1}}{\propto_{1,1}} B_{x}+\left(\propto_{2,2}-\propto_{1,1} \frac{\propto_{1,2}}{\alpha_{1,1}}\right) H_{y}+\left(\propto_{2,3}-\propto_{2,1} \frac{\propto_{1,3}}{\propto_{1,1}}\right) H_{z}\right), \\
& \left\{\frac{\partial E_{y}}{\partial x}-\frac{\partial E_{x}}{\partial y}=i k_{0}\left(\frac{\propto_{\xi, 1}}{\propto_{1,1}} B_{x}+\left(\propto_{\xi, 2}-\propto_{\xi, 1} \frac{\alpha_{1,2}}{\alpha_{1,1}}\right) H_{y}+\left(\propto_{\xi, 3}-\propto_{\xi, 1} \frac{\propto_{1,3}}{\propto_{1,1}}\right) H_{z}\right),\right. \\
& \frac{\partial H_{z}}{\partial y}-\frac{\partial H_{y}}{\partial z}=-i k_{0} D_{x}, \\
& \frac{\partial H_{x}}{\partial z}-\frac{\partial H_{z}}{\partial x}=-i k_{0}\left(\frac{\varepsilon_{2,1}}{\varepsilon_{1,1}} D_{x}+\left(\varepsilon_{2,2}-\varepsilon_{2,1} \frac{\varepsilon_{1,2}}{\varepsilon_{1,1}}\right) E_{y}+\left(\varepsilon_{2,3}-\varepsilon_{2,1} \frac{\varepsilon_{1,3}}{\varepsilon_{1,1}}\right) E_{z}\right), \\
& \frac{\partial H_{y}}{\partial x}-\frac{\partial H_{x}}{\partial y}=-i k_{0}\left(\frac{\varepsilon_{3,1}}{\varepsilon_{1,1}} D_{x}+\left(\varepsilon_{3,2}-\varepsilon_{3,1} \frac{\varepsilon_{1,2}}{\varepsilon_{1,1}}\right) E_{y}+\left(\varepsilon_{3,3}-\varepsilon_{3,1} \frac{\varepsilon_{1,3}}{\varepsilon_{1,1}}\right) E_{z}\right) \text {. }
\end{aligned}
$$

Equations (1.43) do not contain products of functions simultaneously suffering a discontinuity. Therefore, in the transition to the space-frequency domain, we can use the direct Laurent rule (1.29), (1.30). According to (1.42), the vectors of the Fourier coefficients of the functions $D_{x}, B_{x}$ have the form 


$$
\begin{aligned}
& {\left[D_{x}\right]=\llbracket \frac{1}{\varepsilon_{11}} \rrbracket^{-1}\left(\left[E_{x}\right]+\llbracket \frac{\varepsilon_{12}}{\varepsilon_{11}} \rrbracket\left[E_{y}\right]+\llbracket \frac{\varepsilon_{13}}{\varepsilon_{11}} \rrbracket\left[E_{z}\right]\right),} \\
& {\left[B_{x}\right]=\llbracket \frac{1}{\mu_{11}} \rrbracket^{-1}\left(\left[H_{x}\right]+\llbracket \frac{\mu_{12}}{\mu_{11}} \rrbracket\left[H_{y}\right]+\llbracket \frac{\mu_{13}}{\mu_{11}} \rrbracket\left[H_{z}\right]\right) .}
\end{aligned}
$$

Performing in (1.43) the transition to the space-frequency domain and transforming the result, we also obtain the system of differential equations (1.35)-(1.34) where the matrices $\mathbf{M}_{i, j}$ and $\mathbf{E}_{i, j}$ have the form

$$
\begin{aligned}
& \mathbf{M}_{1,1}=\llbracket \frac{1}{\alpha_{11}}\left\|^{-1}, \mathbf{M}_{1,2}=\llbracket \frac{1}{\alpha_{11}} \rrbracket^{-1} \llbracket \frac{\alpha_{12}}{\alpha_{11}} \rrbracket, \quad \mathbf{M}_{1,3}=\llbracket \frac{1}{\alpha_{11}}\right\|^{-1} \llbracket \frac{\alpha_{13}}{\alpha_{11}} \rrbracket,
\end{aligned}
$$

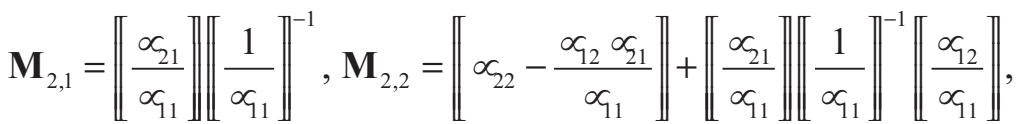

$$
\begin{aligned}
& \mathbf{M}_{2,3}=\llbracket \propto_{23}-\frac{\alpha_{13} \alpha_{21}}{\alpha_{11}} \rrbracket+\llbracket\left[\frac{\alpha_{21}}{\alpha_{11}}\right] \llbracket \| \frac{1}{\alpha_{11}} \rrbracket^{-1} \llbracket \frac{\alpha_{q_{3}}}{\alpha_{q_{1}}} \rrbracket \text {, } \\
& \left.\mathbf{M}_{3,1}=\llbracket \frac{\alpha_{31}}{\propto_{11}}\right] \| \llbracket \frac{1}{\propto_{11}} \rrbracket^{-1}, \mathbf{M}_{3,2}=\llbracket \propto_{32}-\frac{\propto_{31} \propto_{12}}{\propto_{11}} \rrbracket+\llbracket \frac{\alpha_{31}}{\mu_{11}} \rrbracket \llbracket\left[\frac{1}{\mu_{11}} \|^{-1} \llbracket \frac{\mu_{12}}{\mu_{11}} \rrbracket\right. \text {, } \\
& \mathbf{M}_{3,3}=\llbracket \mu_{33}-\frac{\mu_{31} \mu_{13}}{\mu_{11}} \rrbracket+\llbracket \frac{\mu_{31}}{\mu_{11}} \| \llbracket \llbracket \frac{1}{\mu_{11}} \rrbracket^{-1} \llbracket \frac{\mu_{13}}{\mu_{11}} \rrbracket \text {, } \\
& \mathbf{E}_{1,1}=\llbracket \frac{1}{\varepsilon_{11}} \|^{-1}, \quad \mathbf{E}_{1,2}=\llbracket \frac{1}{\varepsilon_{11}} \rrbracket^{-1} \llbracket \frac{\varepsilon_{12}}{\varepsilon_{11}} \rrbracket, \quad \mathbf{E}_{1,3}=\llbracket \frac{1}{\varepsilon_{11}} \rrbracket^{-1} \llbracket \frac{\varepsilon_{13}}{\varepsilon_{11}} \rrbracket, \\
& \left.\mathbf{E}_{2,1}=\llbracket \frac{\varepsilon_{21}}{\varepsilon_{11}} \rrbracket \llbracket\left[\frac{1}{\varepsilon_{11}}\right]^{-1}, \mathbf{E}_{2,2}=\llbracket \varepsilon_{22}-\frac{\varepsilon_{12} \varepsilon_{21}}{\varepsilon_{11}} \rrbracket+\llbracket \frac{\varepsilon_{21}}{\varepsilon_{11}} \rrbracket \llbracket \llbracket \frac{1}{\varepsilon_{11}}\right]^{-1} \llbracket \llbracket \frac{\varepsilon_{12}}{\varepsilon_{11}} \rrbracket, \\
& \left.\mathbf{E}_{2,3}=\llbracket \varepsilon_{23}-\frac{\varepsilon_{13} \varepsilon_{21}}{\varepsilon_{11}} \rrbracket+\llbracket \frac{\varepsilon_{21}}{\varepsilon_{11}}\right] \llbracket\left[\frac{1}{\varepsilon_{11}} \rrbracket^{-1} \llbracket \llbracket \frac{\varepsilon_{13}}{\varepsilon_{11}} \rrbracket,\right. \\
& \left.\mathbf{E}_{3,1}=\llbracket \frac{\varepsilon_{31}}{\varepsilon_{11}}\right] \llbracket \llbracket \frac{1}{\varepsilon_{11}} \rrbracket^{-1}, \mathbf{E}_{3,2}=\llbracket \varepsilon_{32}-\frac{\varepsilon_{31} \varepsilon_{12}}{\varepsilon_{11}} \rrbracket+\llbracket \frac{\varepsilon_{31}}{\varepsilon_{11}} \rrbracket \llbracket \frac{1}{\varepsilon_{11}} \rrbracket^{-1} \llbracket \frac{\varepsilon_{12}}{\varepsilon_{11}} \rrbracket, \\
& \mathbf{E}_{3,3}=\llbracket \varepsilon_{33}-\frac{\varepsilon_{31} \varepsilon_{13}}{\varepsilon_{11}} \rrbracket+\llbracket \frac{\varepsilon_{31}}{\varepsilon_{11}} \rrbracket \llbracket \frac{1}{\varepsilon_{11}} \rrbracket^{-1} \llbracket \frac{\varepsilon_{13}}{\varepsilon_{11}} \rrbracket \text {. }
\end{aligned}
$$


The system of differential equations (1.35) with the matrices $\mathbf{M}_{i, j}, \mathbf{E}_{i, j}$ in the form (1.45) will be called a system, obtained using the correct rules of the Fourier expansions of the product of the functions.

\section{The form of the matrix of the system for different permittivity tensors}

We consider some special kinds of tensors of permittivity and permeability and the corresponding matrices of the system of differential equations (1.35). The systems of differential equations are presented for the case of correct rules of the Fourier expansions.

Consider the case of an isotropic material. For this material, $\mu=1$ and $\varepsilon$ is a scalar. In this case, the matrices $\mathbf{E}_{i, j}, \mathbf{M}_{i, j}$, take the following form:

$$
\begin{aligned}
& \mathbf{E}_{1,1}=\mathbf{E}^{*}=\llbracket \frac{1}{\varepsilon} \rrbracket^{-1}, \mathbf{E}_{2,2}=\mathbf{E}_{3,3}=\mathbf{E}=\llbracket \varepsilon \rrbracket, \\
& \mathbf{E}_{1,2}=\mathbf{E}_{1,3}=\mathbf{E}_{2,1}=\mathbf{E}_{2,3}=\mathbf{E}_{3,1}=\mathbf{E}_{3,2}=\mathbf{0}, \\
& \mathbf{M}_{1,1}=\mathbf{M}_{2,2}=\mathbf{M}_{3,3}=\mathbf{I}, \\
& \mathbf{M}_{1,2}=\mathbf{M}_{1,3}=\mathbf{M}_{2,1}=\mathbf{M}_{2,3}=\mathbf{M}_{3,1}=\mathbf{M}_{3,2}=\mathbf{0},
\end{aligned}
$$

where $\mathbf{I}$ is the identity matrix with the dimensions $(2 N+1) \times(2 N+1)$. Substituting (1.46) into (1.36)-(1.34), we obtain the matrix of the system of differential equations in the form

$$
\mathbf{A}=-\left[\begin{array}{cccc}
\mathbf{0} & \mathbf{0} & \mathbf{K}_{y} \mathbf{E}^{-1} \mathbf{K}_{x} & \mathbf{I}-\mathbf{K}_{y} \mathbf{E}^{-1} \mathbf{K}_{y} \\
\mathbf{0} & \mathbf{0} & \mathbf{K}_{x} \mathbf{E}^{-1} \mathbf{K}_{x}-\mathbf{I} & -\mathbf{K}_{x} \mathbf{E}^{-1} \mathbf{K}_{y} \\
\mathbf{K}_{y} \mathbf{K}_{x} & \mathbf{E}^{*}-\mathbf{K}_{y}^{2} & \mathbf{0} & \mathbf{0} \\
\mathbf{K}_{x}^{2}-\mathbf{E} & -\mathbf{K}_{x} \mathbf{K}_{y} & \mathbf{0} & \mathbf{0}
\end{array}\right]
$$

Consider the case of a planar incidence when $k_{y}=0(\varphi=0)$ in (1.22) and the direction vector of the incident wave lies in the plane $x O z$. In this case, $\mathbf{K}_{y}=\mathbf{0}(1.47)$ and the system of differential equations (1.35) splits into two independent systems

$$
\begin{aligned}
\frac{d}{d z^{\prime}}\left[\begin{array}{l}
\mathbf{S}_{y} \\
\mathbf{U}_{x}
\end{array}\right] & =\mathbf{A}^{\mathrm{TE}}\left[\begin{array}{l}
\mathbf{S}_{y} \\
\mathbf{U}_{x}
\end{array}\right], \mathbf{A}^{\mathrm{TE}}=-\left[\begin{array}{cc}
\mathbf{0} & \mathbf{I} \\
\mathbf{K}_{x}^{2}-\mathbf{E} & \mathbf{0}
\end{array}\right], \\
\frac{d}{d z^{\prime}}\left[\begin{array}{l}
\mathbf{U}_{y} \\
\mathbf{S}_{x}
\end{array}\right] & =\mathbf{A}^{\mathrm{TM}}\left[\begin{array}{l}
\mathbf{U}_{y} \\
\mathbf{S}_{x}
\end{array}\right], \mathbf{A}^{\mathrm{TM}}=-\left[\begin{array}{cc}
\mathbf{0} & \mathbf{E}^{*} \\
\mathbf{K}_{x} \mathbf{E}^{-1} \mathbf{K}_{x}-\mathbf{I} & \mathbf{0}
\end{array}\right] .
\end{aligned}
$$


This result in the case of a planar incidence reduces the solution of the problem of diffraction to two independent problems of diffraction of the waves with TM- and TE-polarization.

Currently, there is considerable interest in the structures comprising layers of a magnetic material. In such structures the optical properties of the materials can be modified by an external magnetic field. This allows to effectively control the amplitude and phase of the diffraction orders with the help of an external magnetic field. For magnetic materials the dielectric constant is given by the tensor $[16,17]$ :

$$
\vec{\varepsilon}=\left[\begin{array}{ccc}
\varepsilon & i g \cos \theta_{M} & -i g \sin \theta_{M} \sin \varphi_{M} \\
-i g \cos \theta_{M} & \varepsilon & i g \sin \theta_{M} \cos \varphi_{M} \\
i g \sin \theta_{M} \sin \varphi_{M} & -i g \sin \theta_{M} \cos \varphi_{M} & \varepsilon
\end{array}\right]
$$

where $\varepsilon$ is the main dielectric constant of the medium, $g$ is the modulus of the gyration vector of the medium proportional to the magnetization $[16,17], \theta_{M}$ and $\varphi_{M}$ are the spherical coordinates, describing the direction of the magnetization vector. In the optical frequency range $\mu=1$.

We consider three basic cases corresponding to the direction of the magnetization vector along the three coordinate axes.

For the polar geometry of magnetization (magnetization vector is perpendicular to the plane of the layers of the structure and directed along the axis $\mathrm{Oz}$ ) $\theta_{M}=0$ and the tensor (1.49) takes the form:

$$
\vec{\varepsilon}=\left[\begin{array}{ccc}
\varepsilon & i g & 0 \\
-i g & \varepsilon & 0 \\
0 & 0 & \varepsilon
\end{array}\right]
$$

In this case

$$
\mathbf{A}=-\left[\begin{array}{cccc}
\mathbf{0} & \mathbf{0} & \mathbf{K}_{y} \mathbf{E}^{-1} \mathbf{K}_{x} & \mathbf{I}-\mathbf{K}_{y} \mathbf{E}^{-1} \mathbf{K}_{y} \\
\mathbf{0} & \mathbf{0} & \mathbf{K}_{x} \mathbf{E}^{-1} \mathbf{K}_{x}-\mathbf{I} & -\mathbf{K}_{x} \mathbf{E}^{-1} \mathbf{K}_{y} \\
i \mathbf{E}^{*} \mathbf{H}+\mathbf{K}_{y} \mathbf{K}_{x} & \mathbf{E}^{*}-\mathbf{K}_{y}^{2} & \mathbf{0} & \mathbf{0} \\
\mathbf{K}_{x}^{2}-\mathbf{E}_{2} & i \mathbf{H} \mathbf{E}^{*}-\mathbf{K}_{x} \mathbf{K}_{y} & \mathbf{0} & \mathbf{0}
\end{array}\right],
$$

where 


$$
\mathbf{E}^{*}=\llbracket \frac{1}{\varepsilon} \rrbracket^{-1}, \mathbf{E}=\llbracket \varepsilon \rrbracket, \quad \mathbf{H}=\llbracket \frac{g}{\varepsilon} \rrbracket, \mathbf{E}_{2}=\llbracket \varepsilon-\frac{g^{2}}{\varepsilon} \rrbracket+\mathbf{H E}^{*} \mathbf{H}
$$

In the case of the meridional geometry of magnetization (the magnetization vector is parallel to the plane of the layers and directed along the axis Ox) $\theta_{M}=\frac{\pi}{2}, \varphi_{M}=0$ and the tensor (1.49) takes the
form

$$
\vec{\varepsilon}=\left[\begin{array}{ccc}
\varepsilon & 0 & 0 \\
0 & \varepsilon & i g \\
0 & -i g & \varepsilon
\end{array}\right]
$$

In the case of (1.52) the matrix of the system takes the form

$$
\mathbf{A}=-\left[\begin{array}{cccc}
\mathbf{K}_{y} \mathbf{E}^{-1} \mathbf{G} & \mathbf{0} & \mathbf{K}_{y} \mathbf{E}^{-1} \mathbf{K}_{x} & \mathbf{I}-\mathbf{K}_{y} \mathbf{E}^{-1} \mathbf{K}_{y} \\
\mathbf{K}_{x} \mathbf{E}^{-1} \mathbf{G} & \mathbf{0} & \mathbf{K}_{x} \mathbf{E}^{-1} \mathbf{K}_{x}-\mathbf{I} & -\mathbf{K}_{x} \mathbf{E}^{-1} \mathbf{K}_{y} \\
\mathbf{K}_{y} \mathbf{K}_{x} & \mathbf{E}^{*}-\mathbf{K}_{y}^{2} & \mathbf{0} & \mathbf{0} \\
\mathbf{G} \mathbf{E}^{-1} \mathbf{G}+\mathbf{K}_{x}^{2}-\mathbf{E} & -\mathbf{K}_{x} \mathbf{K}_{y} & \mathbf{G} \mathbf{E}^{-1} K_{x} & -\mathbf{G E}^{-1} \mathbf{K}_{y}
\end{array}\right],
$$

where $\mathbf{G}=\llbracket g \rrbracket$.

For the equatorial geometry of magnetization (the magnetization vector is parallel to the plane of layers and the direction of the axis Oy) $\theta_{M}=\varphi_{M}=\frac{\pi}{2}$. In this case from (1.49) we obtain:

$$
\vec{\varepsilon}=\left[\begin{array}{ccc}
\varepsilon & 0 & i g \\
0 & \varepsilon & 0 \\
-i g & 0 & \varepsilon
\end{array}\right]
$$

For the tensor (1.54) the matrix of the system takes the form

$$
\mathbf{A}=-\left[\begin{array}{cccc}
\mathbf{0} & \mathbf{K}_{y} \mathbf{E}_{2}^{-1} \mathbf{H} \mathbf{E}^{*} & \mathbf{K}_{y} \mathbf{E}_{2}^{-1} \mathbf{K}_{x} & \mathbf{I}-\mathbf{K}_{y} \mathbf{E}_{2}^{-1} \mathbf{K}_{y} \\
\mathbf{0} & \mathbf{K}_{x} \mathbf{E}_{2}^{-1} \mathbf{H} \mathbf{E}^{*} & \mathbf{K}_{x} \mathbf{E}_{2}^{-1} \mathbf{K}_{x}-\mathbf{I} & -\mathbf{K}_{x} \mathbf{E}_{2}^{-1} \mathbf{K}_{y} \\
\mathbf{K}_{y} \mathbf{K}_{x} & \mathbf{E}^{*}-\mathbf{K}_{y}^{2}-\mathbf{E}^{*} \mathbf{H} \mathbf{E}_{2}^{-1} \mathbf{H} \mathbf{E}^{*} & -\mathbf{E}^{*} \mathbf{H} \mathbf{E}_{2}^{-1} \mathbf{K}_{x} & \mathbf{E}^{*} \mathbf{H} \mathbf{E}_{2}^{-1} \mathbf{K}_{y} \\
\mathbf{K}_{x}^{2}-\mathbf{E} & -\mathbf{K}_{x} \mathbf{K}_{y} & \mathbf{0} & \mathbf{0}
\end{array}\right]
$$




\subsubsection{Representation of the field inside the layer}

For direct representation of the field in the layer, we consider the eigendecomposition of the matrix A:

$$
\mathbf{A}=\mathbf{W} \mathbf{\Lambda} \mathbf{W}^{-1},
$$

wherein $\boldsymbol{\Lambda}=\operatorname{diag} \lambda_{i}$ is the diagonal matrix of eigenvalues of the matrix $\mathbf{A}$, and $\mathbf{W}$ is the matrix of eigenvectors. Then the solution of system of differential equations (1.35) can be written as

$$
\left[\begin{array}{l}
\mathbf{S}_{y} \\
\mathbf{S}_{x} \\
\mathbf{U}_{y} \\
\mathbf{U}_{x}
\end{array}\right]=\mathbf{W} \exp \left(\boldsymbol{\Lambda} z^{\prime}\right) \mathbf{C}^{\prime}=\mathbf{W} \exp \left(\boldsymbol{\Lambda} k_{0} z\right) \mathbf{C}^{\prime}=\sum_{i} c_{i}^{\prime} \mathbf{w}_{i} \exp \left(\lambda_{i} k_{0} z\right)
$$

We split the last sum into two depending on the sign of the real part $\lambda_{i}$ and write the expression in matrix notation:

$$
\begin{aligned}
& {\left[\begin{array}{l}
\mathbf{S}_{y} \\
\mathbf{S}_{x} \\
\mathbf{U}_{y} \\
\mathbf{U}_{x}
\end{array}\right]=\sum_{i} c_{i}^{\prime} \mathbf{w}_{i} \exp \left(\lambda_{i} k_{0} z\right)=\sum_{i: \operatorname{Re} \lambda_{i}<0} c_{i} \mathbf{w}_{i} \exp \left(\lambda_{i} k_{0}\left(z-z_{l}\right)\right)+} \\
& \quad \sum_{i: \operatorname{Re}_{i}>0} c_{i} \mathbf{w}_{i} \exp \left(\lambda_{i} k_{0}\left(z-z_{l-1}\right)\right)= \\
&=\mathbf{W}^{(-)} \exp \left(\boldsymbol{\Lambda}^{(-)} k_{0}\left(z-z_{l}\right)\right) \mathbf{C}^{(-)}+ \\
& \mathbf{W}^{(+)} \exp \left(\boldsymbol{\Lambda}^{(+)} k_{0}\left(z-z_{l-1}\right)\right) \mathbf{C}^{(+)},
\end{aligned}
$$

where $\boldsymbol{\Lambda}^{(+)}$and $\boldsymbol{\Lambda}^{(-)}$are the diagonal matrices of the eigenvalues whose the real parts are positive and negative, respectively, $\mathbf{W}^{(+)}$ and $\mathbf{W}^{(-)}$are the corresponding eigenvector matrices, $\mathbf{C}^{(-)}, \mathbf{C}^{(+)}$are the vectors of arbitrary constants. The representation (1.58) is suitable for numerical calculations. The exponent in (1.58) always has a negative real part. This ensures that there is no numerical overflow.

The procedure for calculating the eigenvectors and eigenvalues can in some cases be significantly speeded up taking into account the specific form of the matrix $\mathbf{A}$ [4]. In particular, the matrix $\mathbf{A}$ in (1.47), (1.48) and (1.51) has the following block structure: 


$$
\mathbf{A}=\left[\begin{array}{cc}
\mathbf{0} & \mathbf{A}_{12} \\
\mathbf{A}_{21} & \mathbf{0}
\end{array}\right]
$$

We write for $\mathbf{A}$ the matrices of eigenvectors and eigenvalues in the form

$$
\mathbf{W}=\left[\begin{array}{ll}
\mathbf{W}_{11} & \mathbf{W}_{12} \\
\mathbf{W}_{21} & \mathbf{W}_{22}
\end{array}\right], \quad \boldsymbol{\Lambda}=\left[\begin{array}{cc}
\boldsymbol{\Lambda}_{11} & \mathbf{0} \\
\mathbf{0} & \boldsymbol{\Lambda}_{22}
\end{array}\right]
$$

Since $\mathbf{A}=\mathbf{W} \mathbf{\Lambda} \mathbf{W}^{-1}$, then

$$
\begin{aligned}
{\left[\begin{array}{cc}
\mathbf{0} & \mathbf{A}_{12} \\
\mathbf{A}_{21} & \mathbf{0}
\end{array}\right] \cdot\left[\begin{array}{ll}
\mathbf{W}_{11} & \mathbf{W}_{12} \\
\mathbf{W}_{21} & \mathbf{W}_{22}
\end{array}\right] } & =\left[\begin{array}{ll}
\mathbf{W}_{11} & \mathbf{W}_{12} \\
\mathbf{W}_{21} & \mathbf{W}_{22}
\end{array}\right] \cdot\left[\begin{array}{cc}
\boldsymbol{\Lambda}_{11} & \mathbf{0} \\
\mathbf{0} & \boldsymbol{\Lambda}_{22}
\end{array}\right] \\
{\left[\begin{array}{ll}
\mathbf{A}_{12} \mathbf{W}_{21} & \mathbf{A}_{12} \mathbf{W}_{22} \\
\mathbf{A}_{21} \mathbf{W}_{11} & \mathbf{A}_{21} \mathbf{W}_{12}
\end{array}\right] } & =\left[\begin{array}{ll}
\mathbf{W}_{11} \boldsymbol{\Lambda}_{11} & \mathbf{W}_{12} \boldsymbol{\Lambda}_{22} \\
\mathbf{W}_{21} \boldsymbol{\Lambda}_{11} & \mathbf{W}_{22} \boldsymbol{\Lambda}_{22}
\end{array}\right]
\end{aligned}
$$

and we have:

$$
\begin{aligned}
& \mathbf{A}_{12} \mathbf{A}_{21} \mathbf{W}_{11}=\mathbf{A}_{12} \mathbf{W}_{21} \boldsymbol{\Lambda}_{11}=\mathbf{W}_{11} \boldsymbol{\Lambda}_{11} \boldsymbol{\Lambda}_{11}, \\
& \mathbf{A}_{12} \mathbf{A}_{21} \mathbf{W}_{12}=\mathbf{A}_{12} \mathbf{W}_{22} \boldsymbol{\Lambda}_{22}=\mathbf{W}_{12} \boldsymbol{\Lambda}_{22} \boldsymbol{\Lambda}_{22}
\end{aligned}
$$

We introduce the matrix $\mathbf{B}=\mathbf{A}_{12} \mathbf{A}_{21}$ and write (1.61) in the form

$$
\mathbf{B} \cdot \mathbf{W}_{11}=\mathbf{W}_{11} \Lambda_{11}^{2}, \quad \mathbf{B} \cdot \mathbf{W}_{12}=\mathbf{W}_{12} \Lambda_{22}^{2} .
$$

According to (1.62) $\mathbf{W}_{11}, \Lambda^{\prime}=\Lambda_{11}^{2}, \mathbf{W}_{12}, \Lambda_{22}^{2}$ are the matrices of the eigenvectors and the diagonal matrices of the eigenvalues of the same matrix B. Therefore

$$
\mathbf{W}_{11}=\mathbf{W}_{12}, \quad \Lambda_{22}=-\boldsymbol{\Lambda}_{11} \text { and } \mathbf{W}_{21}=\mathbf{A}_{21} \mathbf{W}_{11} \boldsymbol{\Lambda}_{11}^{-1}, \quad \mathbf{W}_{22}=-\mathbf{W}_{21} .
$$

The relations (1.63) determine the eigenvalues and eigenvectors of the matrix $\mathbf{A}$ by the eigenvalues and eigenvectors of the matrix B half their size in the form:

$$
\mathbf{W}=\left[\begin{array}{cc}
\mathbf{W}_{11} & \mathbf{W}_{11} \\
\mathbf{A}_{21} \mathbf{W}_{11} \sqrt{\Lambda^{\prime}} & -\mathbf{A}_{21} \mathbf{W}_{11} \sqrt{\Lambda^{\prime}}-1
\end{array}\right], \quad \boldsymbol{\Lambda}=\left[\begin{array}{cc}
\sqrt{\Lambda^{\prime}} & 0 \\
0 & -\sqrt{\Lambda^{\prime}}
\end{array}\right]
$$


In [4] it is pointed out that halving the dimensions of the eigenvalue problem is equivalent to transforming the system (1.35) of $4(2 N+1)$ first-order differential equations to a system $2(2 N+1)$ of second-order differential equations.

\subsubsection{5. 'Stitching' of the electromagnetic field at the}

boundaries of layers

The general representation of the field in the layer was described above. To obtain a solution that satisfies the Maxwell's equations, it is necessary to equate the tangential components of the fields at the boundaries of the layers. Equating the tangential field components is equivalent to equating the functions (1.58) corresponding to the Fourier coefficients at each fixed $z$. We write preliminary solutions of (1.58) on the upper and lower boundaries of all the layers. For convenience, we assume that the matrix of eigenvectors $\mathbf{W}$ is given by:

$$
\mathbf{W}=\left[\begin{array}{ll}
\mathbf{W}^{(-)} & \mathbf{W}^{(+)}
\end{array}\right] .
$$

In addition, we introduce a vector of unknown constants $\mathbf{C}$ as follows:

$$
\mathbf{C}=\left[\begin{array}{l}
\mathbf{C}^{(-)} \\
\mathbf{C}^{(+)}
\end{array}\right] .
$$

In view of this notation the solution of (1.58) on the upper and lower boundaries of the layer has the form

$$
\left[\begin{array}{l}
\mathbf{S}_{y}\left(z_{l-1}\right) \\
\mathbf{S}_{x}\left(z_{l-1}\right) \\
\mathbf{U}_{y}\left(z_{l-1}\right) \\
\mathbf{U}_{x}\left(z_{l-1}\right)
\end{array}\right]=\mathbf{W}^{(-)} \mathbf{X}^{(-)} \mathbf{C}^{(-)}+\mathbf{W}^{(+)} \mathbf{C}^{(+)}=\mathbf{W}\left[\begin{array}{cc}
\mathbf{X}^{(-)} & \mathbf{0} \\
\mathbf{0} & \mathbf{I}
\end{array}\right] \mathbf{C}=\mathbf{N C},
$$

$$
\left[\begin{array}{l}
\mathbf{S}_{y}\left(z_{l}\right) \\
\mathbf{S}_{x}\left(z_{l}\right) \\
\mathbf{U}_{y}\left(z_{l}\right) \\
\mathbf{U}_{x}\left(z_{l}\right)
\end{array}\right]=\mathbf{W}^{(-)} \mathbf{C}^{(-)}+\mathbf{W}^{(+)} \mathbf{X}^{(+)} \mathbf{C}^{(+)}=\mathbf{W}\left[\begin{array}{cc}
\mathbf{I} & \mathbf{0} \\
\mathbf{0} & \mathbf{X}^{(+)}
\end{array}\right] \mathbf{C}=\mathbf{M C},
$$

where

$$
\mathbf{X}^{(+)}=\exp \left(\boldsymbol{\Lambda}^{(+)} k_{0}\left(z_{l}-z_{l-1}\right)\right), \quad \mathbf{X}^{(-)}=\exp \left(-\boldsymbol{\Lambda}^{(-)} k_{0}\left(z_{l}-z_{l-1}\right)\right) .
$$


Equating the tangential components at the interface of the adjacent layers, we obtain the equations

$$
\mathbf{M}_{l-1} \mathbf{C}_{l-1}=\mathbf{N}_{l} \mathbf{C}_{l}, \quad l=2, \ldots, L,
$$

where the index $l=2$ corresponds to the condition for the lower boundary of the upper layer, $l=L$ for the upper boundary of the lower layer.

The relations (1.70) must be supplemented by the conditions of the equality of the tangential components of the field above the structure of (1.20) and at the upper boundary of the 1st layer, as well as the field below the structure (1.21) and at the lower boundary of the $L$-th layer. Given that the components in the field in the region of the layers are represented by segments of the Fourier series with the dimension $2 N+1$, in representations of the field above and under the grating (1.20)-(1.22) we should also take $2 N+1$ waves at $-N \leq i \leq N$.

By adding these relations for the upper and lower boundaries of the diffractive structure, we obtain the following system of linear equations:

$$
\left\{\begin{array}{l}
\mathbf{D}+\mathbf{P}^{(\mathrm{U})} \mathbf{R}=\mathbf{N}_{1} \mathbf{C}_{1}, \\
\mathbf{M}_{l-1} \mathbf{C}_{l-1}=\mathbf{N}_{l} \mathbf{C}_{l}, l=2, \ldots, L, \\
\mathbf{M}_{L} \mathbf{C}_{L}=\mathbf{P}^{(\mathrm{D})} \mathbf{T}
\end{array}\right.
$$

where $\mathbf{R}$ and $\mathbf{T}$ are the vectors of complex amplitudes of the reflected and transmitted orders, respectively. These vectors are of the form:

$$
\mathbf{R}=\left[\begin{array}{l}
\mathbf{R}_{E} \\
\mathbf{R}_{H}
\end{array}\right], \quad \mathbf{T}=\left[\begin{array}{c}
\mathbf{T}_{E} \\
\mathbf{T}_{H}
\end{array}\right],
$$

where $\mathbf{R}_{E}$ and $\mathbf{T}_{E}$ are the vectors of complex amplitudes of the E-waves, $\mathbf{R}_{H}$ and $\mathbf{T}_{H}$ are the vectors of complex amplitudes of the H-waves.

The vector $\mathbf{D}$ in (1.71) represents the incident wave and has the form (see (1.13) for $\theta \rightarrow \pi-\theta$ ):

$$
\mathbf{D}=\left[\begin{array}{c}
\cos \theta \sin \varphi \cdot \boldsymbol{\delta}_{i} \\
\cos \theta \cos \varphi \cdot \boldsymbol{\delta}_{i} \\
-i n_{\mathrm{U}} \cos \varphi \cdot \boldsymbol{\delta}_{i} \\
i n_{\mathrm{U}} \sin \varphi \cdot \boldsymbol{\delta}_{i}
\end{array}\right] \cos \psi+\left[\begin{array}{c}
\cos \varphi \cdot \boldsymbol{\delta}_{i} \\
-\sin \varphi \cdot \boldsymbol{\delta}_{i} \\
i n_{\mathrm{U}} \cos \theta \sin \varphi \cdot \boldsymbol{\delta}_{i} \\
i n_{\mathrm{U}} \cos \theta \cos \varphi \cdot \boldsymbol{\delta}_{i}
\end{array}\right] \sin \psi
$$


where $\boldsymbol{\delta}_{i}$ is the column vector in which only one element, standing in the middle, is nonzero and is equal to one. Column vector $\boldsymbol{\delta}_{i}$ has the dimension $2 N+1$ and vector $\mathbf{D}$ the dimension $4(2 N+1)$.

The matrices $\mathbf{P}^{(\mathrm{U})}$ and $\mathbf{P}^{(\mathrm{D})}(1.71)$ correspond to the reflected and transmitted orders, respectively, and have the form

$$
\mathbf{P}^{(p)}=\left[\begin{array}{cc}
-\cos \Theta^{(p)} \sin \Phi & \cos \boldsymbol{\Phi} \\
-\cos \Theta^{(p)} \cos \boldsymbol{\Phi} & -\sin \boldsymbol{\Phi} \\
-i n_{\mathrm{p}} \cos \boldsymbol{\Phi} & -i n_{\mathrm{p}} \cos \boldsymbol{\Theta}^{(p)} \sin \boldsymbol{\Phi} \\
i n_{\mathrm{p}} \sin \boldsymbol{\Phi} & -i n_{\mathrm{p}} \cos \boldsymbol{\Theta}^{(p)} \cos \boldsymbol{\Phi}
\end{array}\right], \quad p=\mathrm{U}, \mathrm{D}
$$

where $\boldsymbol{\Phi}, \boldsymbol{\Theta}^{(\mathrm{P})}$ are the diagonal matrices of the angles determining the direction of the scattered diffraction orders. They satisfy the following relations:

$$
\begin{gathered}
\sin \boldsymbol{\Phi}=\operatorname{diag}_{i} \frac{k_{y}}{\sqrt{k_{x, i}^{2}+k_{y}^{2}}}, \cos \boldsymbol{\Phi}=\operatorname{diag}_{i} \frac{k_{x, i}}{\sqrt{k_{x, i}^{2}+k_{y}^{2}}}, \\
\cos \boldsymbol{\Theta}^{(\mathrm{U})}=\operatorname{diag}_{i} \frac{k_{z, \mathrm{U}, i}}{k_{0} n_{\mathrm{U}}}, \cos \boldsymbol{\Theta}^{(\mathrm{D})}=\operatorname{diag}_{i} \frac{-k_{z, \mathrm{D}, i}}{k_{0} n_{\mathrm{D}}} .
\end{gathered}
$$

The expressions (1.73)-(1.74) follow directly from the general formulas (1.12) and (1.13) for a plane wave with the form of propagation constants of the orders (1.22) taken into account.

According to (1.71), the solution of the diffraction problem is reduced to solving a system of linear equations. The sequential expression of the coefficients $\mathbf{C}_{l-1}$ in the layer with the index $(l-1)$ through the coefficients $\mathbf{C}_{l-1}$ in the $l$-th layer allows to reduce the system (1.71) to a system of equations for the coefficients $\mathbf{R}$ and $\mathbf{T}$. Indeed, from the last two equations in (1.71), we obtain

$$
\mathbf{M}_{L-1} \mathbf{C}_{L-1}=\mathbf{N}_{L} \mathbf{M}_{L}^{-1} \mathbf{P}^{(\mathrm{D})} \mathbf{T} \text {. }
$$

By substituting (1.77) into the equation with index $l=L-1$ in (1.71) we have

$$
\mathbf{M}_{L-2} \mathbf{C}_{L-2}=\mathbf{N}_{L-1} \mathbf{M}_{L-1}{ }^{-1} \mathbf{N}_{L} \mathbf{M}_{L}^{-1} \mathbf{P}^{(\mathrm{D})} \mathbf{T} .
$$

Continuing this process to the equation with the index $l=2$, we obtain 


$$
\mathbf{M}_{1} \mathbf{C}_{1}=\left(\prod_{l=2}^{L} \mathbf{N}_{l} \mathbf{M}_{l}^{-1}\right) \mathbf{P}^{(\mathrm{D})} \mathbf{T} .
$$

Finally, substituting (1.79) in the first equation (1.71), we obtain the desired system of linear equations for the coefficients $\mathbf{R}$ and $\mathbf{T}$ in the form of:

$$
\mathbf{D}+\mathbf{P}^{(\mathrm{U})} \mathbf{R}=\left(\prod_{l=1}^{L} \mathbf{N}_{l} \mathbf{M}_{l}^{-1}\right) \mathbf{P}^{(\mathrm{D})} \mathbf{T} .
$$

Note that writing the system in this form allows to calculate the vectors $\mathbf{R}$ and $\mathbf{T}$, but the calculation directly from (1.80) can lead to a numerical instability of the problem [3]. There are several numerically stable approaches [3, 18] to calculating the vectors $\mathbf{R}$ and T. We consider the so-called method of the scattering matrix [18].

\subsubsection{The scattering matrix algorithm}

Consider the Rayleigh expansion (1.20), (1.21) of the field above the structure, but instead of one incident wave (corresponding to the zeroth diffraction order), we consider a set of incident waves (respective orders $-N, \ldots, N$ ). Then, the propagation constant can be described by the following expressions:

$$
\begin{gathered}
k_{x, m}=k_{x, 0}+k_{0} \cdot m \frac{\lambda}{d}, m=-N, \ldots, N ; \\
k_{z, m}^{R}=\sqrt{k_{0}^{2} \varepsilon_{\mathrm{U}}-k_{x, m}^{2}} ; \\
k_{z, m}^{I}=-\sqrt{k_{0}^{2} \varepsilon_{\mathrm{U}}-k_{x, m}^{2}},
\end{gathered}
$$

where $k_{z, m}^{R}$ are the propagation constants corresponding to the reflected orders; $k_{z, m}^{I}$ are the propagation constants of the waves incident on the structure from above.

Also, consider a set of waves incident on the structure from the substrate and write similar relations for the orders under the structure:

$$
\begin{gathered}
k_{z, m}^{T}=-\sqrt{k_{0}^{2} \varepsilon_{\mathrm{D}}-k_{x, m}^{2}} ; \\
k_{z, m}^{J}=\sqrt{k_{0}^{2} \varepsilon_{\mathrm{D}}-k_{x, m}^{2}},
\end{gathered}
$$


where $k_{z, m}^{T}$ are the propagation constants corresponding to the transmitted orders; $k_{z, m}^{J}$ are the propagation constants of the waves incident on the structure from the substrate side.

In this case, when in addition to the $2 N+1$ reflected and $2 N+1$ transmitted diffraction orders there are also $2 N+1$ waves incident from above and $2 N+1$ waves incident from below, the calculation of complex amplitudes of diffraction orders consists in the determination of the S-matrix satisfying the equation

$$
\left[\begin{array}{c}
\mathbf{T} \\
\mathbf{R}
\end{array}\right]=\mathbf{S}\left[\begin{array}{c}
\mathbf{I}_{1} \\
\mathbf{J}_{L+1}
\end{array}\right],
$$

where $\mathbf{R}$ and $\mathbf{T}$ are the vectors of the complex amplitudes of the reflected and transmitted diffraction orders, and $\mathbf{I}_{1}$ and $\mathbf{J}_{L+1}$ are the vectors of the complex amplitudes of the waves incident on the structure from the top and the bottom, respectively. The matrix $\mathbf{S}$ in (1.86) is called the scattering matrix. The scattering matrix $\mathbf{S}$ is completely determined by the geometry of the structure, the optical properties of materials and the parameters of the incident radiation.

The difference between $\mathbf{S}$ and the matrix of the system in the previously obtained expression (1.80) is that now it is necessary to consider not one incident wave but a set of waves incident on the structure from both the top and the substrate side. For the incident waves instead of (1.73) we will use the following expression at $p=\mathrm{U}$ :

$$
\mathbf{D}^{(p)}=\left[\begin{array}{cc}
\cos \boldsymbol{\Theta}^{(p)} \sin \boldsymbol{\Phi} & \cos \boldsymbol{\Phi} \\
\cos \boldsymbol{\Theta}^{(p)} \cos \boldsymbol{\Phi} & -\sin \boldsymbol{\Phi} \\
-i n_{p} \cos \boldsymbol{\Phi} & i n_{p} \cos \boldsymbol{\Theta}^{(p)} \sin \boldsymbol{\Phi} \\
i n_{p} \sin \boldsymbol{\Phi} & i n_{p} \cos \boldsymbol{\Theta}^{(p)} \cos \boldsymbol{\Phi}
\end{array}\right]
$$

When $p=\mathrm{D}$ the expression (1.87) describes the Fourier coefficients of the tangential components of the fields $\mathbf{E}$ and $\mathbf{H}$ corresponding to the waves incident on the structure from the 'bottom'.

The system (1.71) will now take the form: 


$$
\left\{\begin{array}{l}
\mathbf{D}^{(\mathrm{U})} \mathbf{I}_{1}+\mathbf{P}^{(\mathrm{U})} \mathbf{R}=\mathbf{N}_{1} \mathbf{C}_{1}, \\
\mathbf{M}_{l-1} \mathbf{C}_{l-1}=\mathbf{N}_{l} \mathbf{C}_{l}, \quad l=2, \ldots, L, \\
\mathbf{M}_{L} \mathbf{C}_{L}=\mathbf{D}^{(D)} \mathbf{J}_{L+1}+\mathbf{P}^{(D)} \mathbf{T},
\end{array}\right.
$$

We introduce the notation

$$
\begin{array}{ll}
\mathbf{I}_{l}=\mathbf{X}_{l-1}^{(+)} \mathbf{C}_{l-1}^{(+)} ; & \mathbf{J}_{l}=\mathbf{X}_{l}^{(-)} \mathbf{C}_{l}^{(-)} ; \\
\mathbf{R}_{l}=\mathbf{C}_{l-1}^{(-)} ; & \mathbf{T}_{l}=\mathbf{C}_{l}^{(+)},
\end{array}
$$

having the meaning of the complex amplitudes of the incident and scattered orders in each layer (see Fig. 1.2). Furthermore, to write system (1.88) in the most compact form, we introduce the notation

$$
\begin{array}{ll}
\mathbf{C}_{0}^{(-)}=\mathbf{R}_{1}=\mathbf{R} ; & \mathbf{C}_{L+1}^{(+)}=\mathbf{T}_{L+1}=\mathbf{T} ; \\
\mathbf{X}_{0}^{(+)} \mathbf{C}_{0}^{(+)}=\mathbf{I}_{1} ; & \mathbf{X}_{L+1}^{(-)} \mathbf{C}_{L+1}^{(-)}=\mathbf{J}_{L+1} ; \\
\mathbf{W}_{0}^{(-)}=\mathbf{P}^{(\mathrm{U})} ; & \mathbf{W}_{L+1}^{(-)}=\mathbf{D}^{(\mathrm{D})} ; \\
\mathbf{W}_{0}^{(+)}=\mathbf{D}^{(\mathrm{U})} ; & \mathbf{W}_{L+1}^{(+)}=\mathbf{P}^{(\mathrm{D})} .
\end{array}
$$

Then the system $(1.88)$ can be written as

$$
\left\{\mathbf{W}_{l-1}^{(-)} \mathbf{R}_{l}+\mathbf{W}_{l-1}^{(+)} \mathbf{I}_{l}=\mathbf{W}_{l}^{(-)} \mathbf{J}_{l}+\mathbf{W}_{l}^{(+)} \mathbf{T}_{l}, \quad l=1, \ldots, L+1 .\right.
$$

$$
\begin{aligned}
& l-1 \\
& \frac{\boldsymbol{\Phi}_{1}=\mathbf{W}_{l-1}^{(-)}}{\boldsymbol{\Phi}_{2}=\mathbf{W}_{l}^{(-)} \underbrace{\mathbf{X}_{l}^{(-)} \mathbf{C}_{l}^{(-)}}_{\mathbf{C}_{l-1}^{(-)}}+\mathbf{W}_{l-1}^{(+)} \overbrace{\mathbf{X}_{l}^{(+)} \mathbf{X}_{l-1}^{(+)} \mathbf{C}_{l-1}^{(+)}}^{\mathbf{C}_{l}^{(+)}}} \\
& l \\
& \frac{\mathbf{\Phi}_{3}=\mathbf{W}_{l}^{(-)}}{\boldsymbol{\Phi}_{4}=\mathbf{W}_{l+1}^{(-)} \underbrace{\mathbf{X}_{l+1}^{(-)} \mathbf{C}_{l+1}^{(-)}}_{\mathbf{C}_{l}^{(-)}}+\mathbf{W}_{l}^{(+)} \overbrace{\mathbf{J}_{l+1}^{(+)}}^{\mathbf{X}_{l}^{(+)} \mathbf{C}_{l}^{(+)}}} \\
& l+1 \\
& \underbrace{\mathbf{R}_{l+1}}_{\mathbf{T}_{l+1}^{(+)}} \searrow
\end{aligned}
$$

Fig. 1.2. Representation of fields at the bottom border of the $l-1$-th layer $\left(\boldsymbol{\Phi}_{1}\right)$, at the upper $\left(\boldsymbol{\Phi}_{2}\right)$ and lower $\left(\boldsymbol{\Phi}_{3}\right)$ borders of the $l$-th layer and the upper border of the $l+1$-th layer $\left(\boldsymbol{\Phi}_{4}\right)$. 
We consider the numerically stable method for finding the scattering matrix of the multilayer structure [18]. We introduce the notation $\mathbf{S}^{(l)}$ for the scattering matrix, which connects the Fourier components of the field at the lower boundary of the structure and the lower boundary of the $l$-th layer

$$
\left[\begin{array}{ll}
\mathbf{S}_{1,1}^{(l)} & \mathbf{S}_{1,2}^{(l)} \\
\mathbf{S}_{2,1}^{(l)} & \mathbf{S}_{2,2}^{(l)}
\end{array}\right]\left[\begin{array}{c}
\mathbf{I}_{l+1} \\
\mathbf{J}_{L+1}
\end{array}\right]=\left[\begin{array}{c}
\mathbf{T}_{L+1} \\
\mathbf{R}_{l+1}
\end{array}\right]
$$

Also, we introduce the scattering matrix $\tilde{\mathbf{S}}^{(l)}$ linking the Fourier components of the field at the lower boundary of the structure and the upper boundary of $l$ th layer:

$$
\left[\begin{array}{cc}
\tilde{\mathbf{S}}_{1,1}^{(l)} & \tilde{\mathbf{S}}_{1,2}^{(l)} \\
\tilde{\mathbf{S}}_{2,1}^{(l)} & \tilde{\mathbf{S}}_{2,2}^{(l)}
\end{array}\right]\left[\begin{array}{c}
\mathbf{T}_{l} \\
\mathbf{J}_{L+1}
\end{array}\right]=\left[\begin{array}{c}
\mathbf{T}_{L+1} \\
\mathbf{J}_{l}
\end{array}\right] .
$$

Consider the sequential method of constructing the scattering matrix structure, starting from the matrix $\tilde{\mathbf{S}}^{(L+1)}=\mathbf{I}$, where $\mathbf{I}$ is the identity matrix. We consistently find the matrices $\mathbf{S}^{(l)}$ and $\tilde{\mathbf{S}}^{(l)}$ in the following sequence:

$$
\mathbf{I}=\tilde{\mathbf{S}}^{(L+1)} \rightarrow \mathbf{S}^{(L)} \rightarrow \tilde{\mathbf{S}}^{(L)} \rightarrow \mathbf{S}^{(L-1)} \rightarrow \cdots \rightarrow \tilde{\mathbf{S}}^{(1)} \rightarrow \mathbf{S}^{(0)}=\mathbf{S}
$$

Equalities (1.89) make it easy to find the matrix $\tilde{\mathbf{S}}^{(l)}$, knowing the matrix $\mathbf{S}^{(l)}$ :

$$
\tilde{\mathbf{S}}^{(l)}=\left[\begin{array}{cc}
\mathbf{I} & \mathbf{0} \\
\mathbf{0} & \mathbf{X}_{l}^{(-)}
\end{array}\right] \mathbf{S}^{(l)}\left[\begin{array}{cc}
\mathbf{X}_{l}^{(+)} & \mathbf{0} \\
\mathbf{0} & \mathbf{I}
\end{array}\right] .
$$

We find the numerically stable equations for calculating the matrix $\mathbf{S}^{(l-1)}$ on the basis of the matrix $\tilde{\mathbf{S}}^{(l)}$. To do this, we write the equation of the system (1.91) with the number $l+1$ in the following matrix form:

$$
\left[\begin{array}{ll}
\mathbf{W}_{l}^{(+)} & -\mathbf{W}_{l+1}^{(-)}
\end{array}\right]\left[\begin{array}{l}
\mathbf{I}_{l+1} \\
\mathbf{J}_{l+1}
\end{array}\right]=\left[\begin{array}{ll}
\mathbf{W}_{l+1}^{(+)} & -\mathbf{W}_{l}^{(-)}
\end{array}\right]\left[\begin{array}{c}
\mathbf{T}_{l+1} \\
\mathbf{R}_{l+1}
\end{array}\right]
$$

We introduce the notation $\mathbf{H}^{(l)}$ by rewriting the expression (1.96) as follows: 


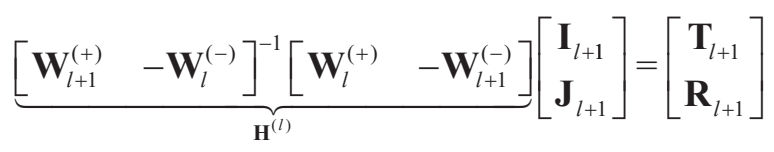

Matrix $\mathbf{H}^{(l)}$ has the meaning of the scattering matrix linking the field at the lower boundary of the $l$-th layer and the upper boundary of the $l+1$-th layer. On the basis of expressions (1.92), (1.93), (1.97) it can be shown that the matrix $\mathbf{S}^{(l)}$ is calculated as

$$
\mathbf{S}^{(l)}=\mathbf{H}^{(l)} \otimes \tilde{\mathbf{S}}^{(l+1)},
$$

where the associative operation " $\otimes$ " is defined as follows [18]:

$$
\begin{aligned}
{\left[\begin{array}{ll}
\mathbf{H}_{1,1} & \mathbf{H}_{1,2} \\
\mathbf{H}_{2,1} & \mathbf{H}_{2,2}
\end{array}\right] \otimes\left[\begin{array}{ll}
\mathbf{S}_{1,1} & \mathbf{S}_{1,2} \\
\mathbf{S}_{2,1} & \mathbf{S}_{2,2}
\end{array}\right]=} & \\
& =\left[\begin{array}{cc}
\mathbf{S}_{1,1}\left(\mathbf{I}-\mathbf{H}_{1,2} \mathbf{S}_{2,1}\right)^{-1} \mathbf{H}_{1,1} & \mathbf{S}_{1,2}+\mathbf{S}_{1,1} \mathbf{H}_{1,2}\left(\mathbf{I}-\mathbf{S}_{2,1} \mathbf{H}_{1,2}\right)^{-1} \mathbf{S}_{2,2} \\
\mathbf{H}_{2,1}+\mathbf{H}_{2,2} \mathbf{S}_{2,1}\left(\mathbf{I}-\mathbf{H}_{1,2} \mathbf{S}_{2,1}\right)^{-1} \mathbf{H}_{1,1} & \mathbf{H}_{2,2}\left(\mathbf{I}-\mathbf{S}_{2,1} \mathbf{H}_{1,2}\right)^{-1} \mathbf{S}_{2,2}
\end{array}\right] .
\end{aligned}
$$

Note that in (1.95), (1.97), (1.99) there is no inversion of the ill-conditioned matrices, therefore, this method allows to find the scattering matrix of the structure $\mathbf{S}$ and to avoid problems with numerical stability.

If a single plane wave of unit intensity is incident on the structure from above, complex transmission $\mathbf{T}$ and reflection $\mathbf{R}$ coefficients are found from (1.86):

$$
\left[\begin{array}{l}
\mathbf{T} \\
\mathbf{R}
\end{array}\right]=\mathbf{S}\left[\begin{array}{c}
1 \cdot \boldsymbol{\delta}_{i} \\
0 \cdot \boldsymbol{\delta}_{i}
\end{array}\right]
$$

\subsubsection{Calculation of the field distribution}

In the calculation of the field distribution in the structure there may be the same problems with numerical stability as in the calculation of the complex amplitudes of diffraction orders. To construct the distribution of the field on the basis of expressions (1.27) and (1.58), it is necessary to know the values of the vectors $\mathbf{C}^{(-)}, \mathbf{C}^{(+)}$. Consider an iterative procedure for numerically-stable calculation of these vectors [19].

We assume that the vector $\mathbf{R}$ of the complex amplitudes of the reflected orders and matrices $\mathbf{S}^{(l)}, \tilde{\mathbf{S}}^{(l)}$ is already calculated by the method described in the previous section. 
We construct a recursive procedure for computing $\mathbf{C}_{l}^{(+)}$. From (1.97) at $l=0$ we obtain the expression $\mathbf{C}_{1}^{(+)}$in the form:

$$
\mathbf{C}_{1}^{(+)}=\mathbf{T}_{1}=\mathbf{H}_{1,1}^{(0)} \mathbf{I}_{1}+\mathbf{H}_{1,2}^{(0)} \mathbf{J}_{1}=\mathbf{H}_{1,1}^{(0)} \mathbf{I}_{1}+\mathbf{H}_{1,2}^{(0)}\left(\mathbf{H}_{2,2}^{(0)}\right)^{-1}\left(\mathbf{R}-\mathbf{H}_{2,1}^{(0)} \mathbf{I}_{1}\right) .
$$

Now, assuming that $\mathbf{C}_{l}^{(+)}$is known we find expression $\mathbf{C}_{l+1}^{(+)}=\mathbf{T}_{l+1}$. From equation (1.93), taking into account $\mathbf{J}_{L+1}=0$, we obtain

$$
\mathbf{T}_{l+1}=\mathbf{C}_{l+1}^{(+)}=\left(\tilde{\mathbf{S}}_{1,1}^{(l+1)}\right)^{-1} \mathbf{T}_{L+1}=\left(\tilde{\mathbf{S}}_{1,1}^{(l+1)}\right)^{-1} \mathbf{T} .
$$

We write the matrix $\mathbf{S}_{1,1}^{(l)}$ equation of (1.98) (1.99) in the form:

$$
\mathbf{S}_{1,1}^{(l)}=\tilde{\mathbf{S}}_{1,1}^{(l+1)}\left(\mathbf{I}-\mathbf{H}_{1,2}^{(l)} \tilde{\mathbf{S}}_{2,1}^{(l+1)}\right)^{-1} \mathbf{H}_{1,1}^{(l)}
$$

We express the matrix $\tilde{\mathbf{S}}_{1,1}^{(l)}$ from formula (1.95):

$$
\tilde{\mathbf{S}}_{1,1}^{(l)}=\mathbf{S}_{1,1}^{(l)} \mathbf{X}_{l}^{(+)} .
$$

We express from equations (1.103) the matrix $\left(\tilde{\mathbf{S}}_{1,1}^{(l+1)}\right)^{-1}$, and using the equation (1.104) we exclude matrix $\mathbf{S}_{1,1}^{(l)}$ :

$$
\left(\tilde{\mathbf{S}}_{1,1}^{(l+1)}\right)^{-1}=\left(\mathbf{I}-\mathbf{H}_{1,2}^{(l)} \tilde{\mathbf{S}}_{2,1}^{(l+1)}\right)^{-1} \mathbf{H}_{1,1}^{(l)}\left(\mathbf{S}_{1,1}^{(l)}\right)^{-1}=\left(\mathbf{I}-\mathbf{H}_{1,2}^{(l)} \tilde{\mathbf{S}}_{2,1}^{(l+1)}\right)^{-1} \mathbf{H}_{1,1}^{(l)} \mathbf{X}_{l}^{(+)}\left(\tilde{\mathbf{S}}_{1,1}^{(l)}\right)^{-1} .
$$

Multiplying the left and right hand sides of (1.105) on the right by $\mathbf{T}$, with (1.102) taken into account, we finally obtain:

$$
\mathbf{C}_{l+1}^{(+)}=\mathbf{T}_{l+1}=\left(\mathbf{I}-\mathbf{H}_{1,2}^{(l)} \tilde{\mathbf{S}}_{2,1}^{(l+1)}\right)^{-1} \mathbf{H}_{1,1}^{(l)} \mathbf{X}_{l}^{(+)} \mathbf{C}_{l}^{(+)} .
$$

The recurrence relation (1.106) with the formula (1.101) allows to find $\mathbf{C}_{l}^{(+)}$for all $l$. We now obtain a formula for finding $\mathbf{C}_{l}^{(-)}=\mathbf{R}_{l+1}$. From formula (1.92) at $\mathbf{J}_{L+1}=0$ we have:

$$
\mathbf{C}_{l}^{(-)}=\mathbf{R}_{l+1}=\mathbf{S}_{2,1}^{(l)} \mathbf{I}_{l+1}=\mathbf{S}_{2,1}^{(l)} \mathbf{X}_{l}^{(+)} \mathbf{C}_{l}^{(+)} .
$$

\subsubsection{Intensities of the diffraction orders}

In the analysis of the field away from the grating researchers are usually not interested in complex amplitudes (1.72) and pay attention to the intensities of the reflected and transmitted propagating 
diffraction orders. The propagating orders are determined by the real values $k_{z, p, m}$ in (1.22). The intensities of the diffraction orders are defined as the flux of the Umov-Poynting vector through the plane $z=$ const, normalized to the correspondng flux of the incident wave [15]. Taking into account the expressions (1.16) and (1.17) the intensity of the orders can be found from the following expressions:

$$
\begin{gathered}
\mathbf{I}^{R}=\left|\frac{n_{\mathrm{U}} \operatorname{Re}\left(\cos \Theta^{(\mathrm{U})}\right)}{n_{\mathrm{U}} \cos \theta}\right|\left(\left|\mathbf{R}_{E}\right|^{2}+\left|\mathbf{R}_{H}\right|^{2}\right) ; \\
\mathbf{I}^{T}=\left|\frac{n_{\mathrm{D}} \operatorname{Re}\left(\cos \Theta^{(\mathrm{D})}\right)}{n_{\mathrm{U}} \cos \theta}\right|\left(\left|\mathbf{T}_{E}\right|^{2}+\left|\mathbf{T}_{H}\right|^{2}\right),
\end{gathered}
$$

where the diagonal matrices $\cos \Theta^{(\mathrm{U})}, \cos \Theta^{(\mathrm{D})}$ are defined in (1.76), and squaring of vectors $\mathbf{R}_{E}, \mathbf{R}_{H}, \mathbf{T}_{E}, \mathbf{T}_{H}$ is performed element wise. For evanescent diffraction orders $\operatorname{Re}\left(\cos \Theta^{(p)}\right)=0, p=\mathrm{U}, \mathrm{D}$, therefore their intensities are equal to zero.

In general, propagating diffraction orders are plane waves with elliptical polarization. Indeed, each diffraction order corresponds to the superposition of the E- and H-waves. Let $E_{E}, E_{H}$ be the complex amplitude of the electric field at the $\mathrm{E}$ - and $\mathrm{H}$-waves. Note that the electric field vectors in the $\mathrm{E}$ - and $\mathrm{H}$-waves are perpendicular to each other and perpendicular to the direction of wave propagation. The addition of perpendicular oscillations results in the formation of an elliptically polarized wave. The polarization ellipse is characterized by two parameters: the angle $\phi$ of the major axis of the polarization ellipse and the ellipticity parameter $\chi[20]$. The ellipticity parameter characterizes the ratio of the lengths of the $a, b$ axes of the polarization ellipse in the form $\operatorname{tg} \chi=a / b$. The parameters $\phi$ and $\chi$ are determined through complex amplitudes $E_{E}, E_{H}$ as [20]

$$
\begin{aligned}
& \operatorname{tg}(2 \phi)=\frac{2 \operatorname{Re}\left(E_{E} / E_{H}\right)}{1-\left|E_{E} / E_{H}\right|^{2}}, \\
& \sin (2 \chi)=\frac{2 \operatorname{Im}\left(E_{E} / E_{H}\right)}{1+\left|E_{E} / E_{H}\right|^{2}} .
\end{aligned}
$$

In conclusion, let us make a few remarks about the choice of the parameter $N$ that determines the length of the segments of the Fourier series approximating the components of the electric and magnetic 
fields in the grating. For a given $N$ the number of calculated orders is equal to $2 N+1$, from $-N$ to $+N$. The parameter $N$ must be greater than the number of propagating orders. If the grating consists only of dielectrics (all refractive indices are real numbers), and the parameter $N$ satisfies this condition, the energy conservation law in the following form should be satisfied:

$$
\sum I^{R}+\sum I^{T}=1
$$

If the grating contains absorbing materials, the sum (1.111) should be less than one. In general, $N$ is selected in computational experiment on the basis of the condition of stabilizing the intensities of the orders.

\subsubsection{Numerical example}

Consider the example of calculation of the intensities of diffraction orders of a binary grating. The grating geometry is shown in Fig. 1.3. The grating parameters are shown in the Fig. caption.

In the calculations the parameter $N$ that determines the length of the segments of the Fourier series was $N=20$. The calculations were carried out for the TM-polarized normally incident wave. The results of calculation of the reflection and transmission spectra of the grating (the intensities of diffraction orders) are shown in Fig. 1.4 .

\subsubsection{The Fourier modal method for three-dimensional periodic structures}

Consider the described method in the case of three-dimensional periodic diffraction structures. The $z$-axis is perpendicular to the plane in which the diffraction grating is positioned. The functions of permittivity and magnetic permeability in the grating region are assumed to be periodic with respect to the variables $x, y$ with periods $d_{x}$ and $d_{y}$ respectively. As in the two-dimensional case, we assume

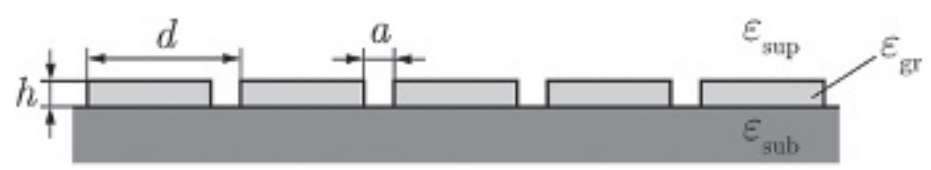

Fig. 1.3. The geometry of the binary grating (Parameters: $d=1000 \mathrm{~nm}, a=$ $\left.200 \mathrm{~nm}, h=200 \mathrm{~nm}, \varepsilon_{\text {sup }}=1, \varepsilon_{\mathrm{gr}}=4, \varepsilon_{\text {sub }}=2.25\right)$. 

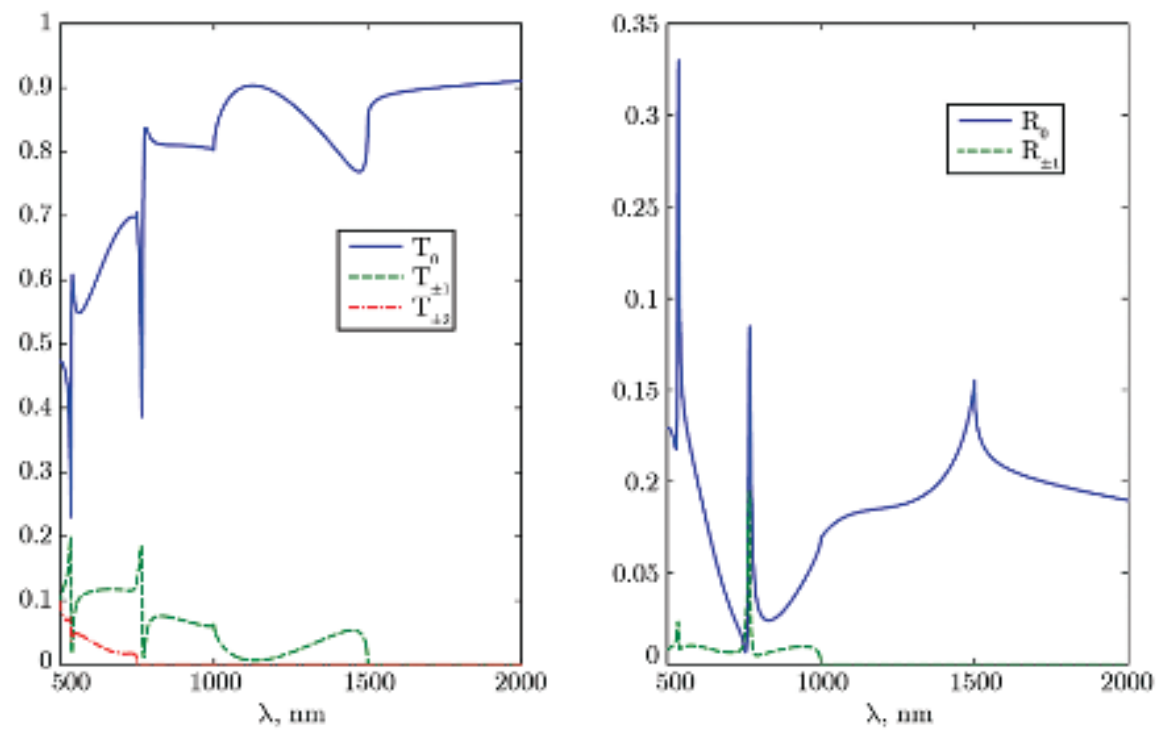

Fig. 1.4. The intensities of the reflected and transmitted diffraction orders.

that the diffraction grating is made up of $L$ binary layers and the permittivity and permeability in each layer are independent of the variable $z$.

The method of solving the problem of diffraction in the threedimensional case is similar to the two-dimensional case considered previously. The main details of the three-dimensional problem are given below.

Upon the plane wave diffraction on a three-dimensional diffraction grating, a set of reflected and transmitted diffraction orders is formed. In this case the field above and below the structure is as follows:

$$
\begin{gathered}
\boldsymbol{\Phi}^{\mathrm{U}}(x, y, z)=\boldsymbol{\Phi}^{\mathrm{inc}}(x, y, z)+\sum_{n} \sum_{m} \boldsymbol{\Phi}_{n, m}^{R}\left(R_{n, m}\right) \exp \left(i\left(k_{x, n} x+k_{y, m} y+k_{z, \mathrm{U}, n, m} z\right)\right), \\
\boldsymbol{\Phi}^{D}(x, y, z)=\sum_{n} \sum_{m} \boldsymbol{\Phi}_{n, m}^{T}\left(T_{n, m}\right) \exp \left(i\left(k_{x, n} x+k_{y, m} y-k_{z, \mathrm{D}, n, m}\left(z-z_{L}\right)\right)\right),
\end{gathered}
$$

where $\boldsymbol{\Phi}^{\mathrm{inc}}(x, y, z)$ is the incident wave. The incident wave is assumed to be given in the form (1.19). The propagation constants of the diffraction orders with numbers $(n, m)$ are described as follows: 


$$
\begin{aligned}
& k_{x, n}=k_{0}\left(n_{\mathrm{U}} \sin \theta \cos \varphi+n \frac{\lambda}{d_{x}}\right), \\
& k_{y, m}=k_{0}\left(n_{\mathrm{U}} \sin \theta \sin \varphi+m \frac{\lambda}{d_{y}}\right), \\
& k_{z, p, n, m}=\sqrt{\left(k_{0} n_{p}\right)^{2}-k_{x, n}^{2}-k_{y, m}^{2}},
\end{aligned}
$$

where as $p$, ' $U$ ' is taken for the reflected orders (the fields above the grating) and ' $\mathrm{D}$ ' - for the transmitted ones (the field below the grating). The form of the propagation constants ensures that the twodimensional quasi-periodicity condition is fulfilled:

$$
\boldsymbol{\Phi}^{p}\left(x+d_{x}, y+d_{y}, z\right)=\boldsymbol{\Phi}^{p}(x, y, z) \exp \left(i k_{x, 0} d_{x}+i k_{y, 0} d_{y}\right), \quad p=\mathrm{U}, \mathrm{D} .
$$

According to (1.115), the amplitude of the field does not change upon a shift along the $x$ and $y$ axes by integer multiples of the corresponding periods. The waves with the real $k_{z, p, n, m}$ are propagating, those with the imaginary value are evanescent.

The electromagnetic field in each layer, as in the two-dimensional case, is described by the basic Maxwell's equations for the monochromatic field in the form (1.24)-(1.27). We represent the components of the electric and magnetic fields in the form of a twodimensional Fourier series in the variables $x, y$ :

$$
\left\{\begin{array}{l}
E_{x}=\sum_{n} \sum_{m} S_{x, n, m}(z) \exp \left(i\left(k_{x, n} x+k_{y, m} y\right)\right), \\
E_{y}=\sum_{n} \sum_{m} S_{y, n, m}(z) \exp \left(i\left(k_{x, n} x+k_{y, m} y\right)\right), \\
E_{z}=\sum_{n} \sum_{m} S_{z, n, m}(z) \exp \left(i\left(k_{x, n} x+k_{y, m} y\right)\right), \\
H_{x}=-i \sum_{n} \sum_{m} U_{x, n, m}(z) \exp \left(i\left(k_{x, n} x+k_{y, m} y\right)\right), \\
H_{y}=-i \sum_{n} \sum_{m} U_{y, n, m}(z) \exp \left(i\left(k_{x, n} x+k_{y, m} y\right)\right), \\
H_{z}=-i \sum_{n} \sum_{m} U_{z, n, m}(z) \exp \left(i\left(k_{x, n} x+k_{y, m} y\right)\right) .
\end{array}\right.
$$

The equations (1.116) are written with the quasi-periodicity of the field components in the variables $x, y$ taken into account. We restrict ourselves to a finite number of terms in the expansions 
(1.116), corresponding to $-N_{x} \leq n \leq N_{x},-N_{y} \leq m \leq N_{y}$. Substituting the expansion (1.116) into (1.26) and equating the coefficients of the same Fourier harmonics, we obtain a system of differential equations in the form

$$
\begin{cases}i k_{0} \mathbf{K}_{y} \mathbf{S}_{z}-\frac{d \mathbf{S}_{y}}{d z} & =k_{0}\left(\mathbf{M}_{1,1} \mathbf{U}_{x}+\mathbf{M}_{1,2} \mathbf{U}_{y}+\mathbf{M}_{1,3} \mathbf{U}_{z}\right), \\ \frac{d \mathbf{S}_{x}}{d z}-i k_{0} \mathbf{K}_{x} \mathbf{S}_{z} & =k_{0}\left(\mathbf{M}_{2,1} \mathbf{U}_{x}+\mathbf{M}_{2,2} \mathbf{U}_{y}+\mathbf{M}_{2,3} \mathbf{U}_{z}\right) \\ i k_{0} \mathbf{K}_{x} \mathbf{S}_{y}-i k_{0} \mathbf{K}_{y} \mathbf{S}_{x} & =k_{0}\left(\mathbf{M}_{3,1} \mathbf{U}_{x}+\mathbf{M}_{3,2} \mathbf{U}_{y}+\mathbf{M}_{3,3} \mathbf{U}_{z}\right) \\ i k_{0} \mathbf{K}_{y} \mathbf{U}_{z}-\frac{d \mathbf{U}_{y}}{d z} & =k_{0}\left(\mathbf{E}_{1,1} \mathbf{S}_{x}+\mathbf{E}_{1,2} \mathbf{S}_{y}+\mathbf{E}_{1,3} \mathbf{S}_{z}\right) \\ \frac{d \mathbf{U}_{x}}{d z}-i k_{0} \mathbf{K}_{x} \mathbf{U}_{z} & =k_{0}\left(\mathbf{E}_{2,1} \mathbf{S}_{x}+\mathbf{E}_{2,2} \mathbf{S}_{y}+\mathbf{E}_{2,3} \mathbf{S}_{z}\right), \\ i k_{0} \mathbf{K}_{x} \mathbf{U}_{y}-i k_{0} \mathbf{K}_{y} \mathbf{U}_{x} & =k_{0}\left(\mathbf{E}_{3,1} \mathbf{S}_{x}+\mathbf{E}_{3,2} S_{y}+\mathbf{E}_{3,3} \mathbf{S}_{z}\right)\end{cases}
$$

The form of the resulting system is the same as that of the system (1.31) for the two-dimensional case. The difference is in the details of the representation of vectors and matrices in the system. The vectors $\mathbf{S}_{x}, \mathbf{S}_{y}, \mathbf{S}_{z}, \mathbf{U}_{x}, \mathbf{U}_{y}, \mathbf{U}_{z}$ in (1.117) are a row-wise onedimensional representation of the matrices $\mathrm{S}_{x, j, k}, \mathrm{~S}_{y, j, k}, \mathrm{~S}_{z, j, k}, \mathrm{U}_{x, j, k}, \mathrm{U}_{y, j, k}$, $\mathrm{U}_{z, j, k},-N_{x} \leq j \leq N_{x},-N_{y} \leq k \leq N_{y}$. This means that the element of the vector $\mathbf{S}_{x}$ with the number

$$
l(i, j)=i\left(2 N_{y}+1\right)+j .
$$

corresponds to the value $\mathbf{S}_{x, i, j}$. The vectors introduced in this manner have the dimension $\left(2 N_{x}+1\right)\left(2 N_{y}+1\right)$ equal to the total number of the calculated diffraction orders. For example, the vector $\mathbf{S}_{x}$ has the form

$$
\mathbf{S}_{x}=\left[S_{x,-N_{x},-N_{y}}, S_{x,-N_{x}, 1-N_{y}}, \ldots, S_{x,-N_{x}, N_{y}}, S_{x, 1-N_{x},-N_{y}}, S_{x, 1-N_{x}, 1-N_{y}}, \ldots, S_{x, N_{x}, N_{y}}\right]^{T}
$$

The matrices $\mathbf{K}_{x}, \mathbf{K}_{y}, \mathbf{E}_{i, j}, \mathbf{M}_{i, j}$ in (1.117) have the dimensions $\left(2 N_{x}+1\right)\left(2 N_{y}+1\right) \times\left(2 N_{x}+1\right)\left(2 N_{y}+1\right)$. The matrices $\mathbf{K}_{x}$ and $\mathbf{K}_{y}$ are defined by the following expressions:

$$
\begin{aligned}
& K_{x, l(i, j), l(n, m)}=k_{x, i} \delta_{i-n} \delta_{j-m} / k_{0}, \\
& K_{y, l(i, j), l(n, m)}=k_{y, j} \delta_{i-n} \delta_{j-m} / k_{0},
\end{aligned}
$$


where $-N_{x} \leq i, n \leq N_{x},-N_{y} \leq j, m \leq N_{y}$.

Consider the preliminary form of the matrices $\mathbf{E}_{i, j}, \mathbf{M}_{i, j}$ obtained by using the direct Laurent rules for the expansion of the product of the function into a Fourier series. The matrices $\mathbf{E}_{i, j}, \mathbf{M}_{i, j}$ consist of the Fourier coefficients of the permittivity and permeability tensors, the structure of the matrices is the same and has the form

$$
T_{l(i, j), l(n, m)}=e_{i-n, j-m},
$$

where $e_{i, j}$ are the Fourier coefficients $-N_{x} \leq i, n \leq N_{x},-N_{y} \leq j, m \leq N_{y}$.

Since the form of the systems of differential equations in the twodimensional and three-dimensional cases is identical, all subsequent changes are also identical. The system of differential equations for the vectors $\mathbf{S}_{x}, \mathbf{S}_{y}, \mathbf{U}_{x}, \mathbf{U}_{y}$, in (1.117) also has the form (1.35)-(1.34). In particular, for a grating of an isotropic material $\varepsilon=\varepsilon(x, y)$ and $\mu=1$ are the scalars, and the matrix of the differential equation system (1.35) has the form

$$
\mathbf{A}=-\left[\begin{array}{cccc}
\mathbf{0} & \mathbf{0} & \mathbf{K}_{y} \mathbf{E}^{-1} \mathbf{K}_{x} & \mathbf{I}-\mathbf{K}_{y} \mathbf{E}^{-1} \mathbf{K}_{y} \\
\mathbf{0} & \mathbf{0} & \mathbf{K}_{x} \mathbf{E}^{-1} \mathbf{K}_{x}-\mathbf{I} & -\mathbf{K}_{x} \mathbf{E}^{-1} \mathbf{K}_{y} \\
\mathbf{K}_{y} \mathbf{K}_{x} & \mathbf{E}-\mathbf{K}_{y}^{2} & \mathbf{0} & \mathbf{0} \\
\mathbf{K}_{x}^{2}-\mathbf{E} & -\mathbf{K}_{x} \mathbf{K}_{y} & \mathbf{0} & \mathbf{0}
\end{array}\right]
$$

where the matrix $\mathbf{E}$ has the form (1.120) and is composed of the Fourier coefficients of functions $\varepsilon(x, y)$. The formula (1.122) is obtained from the general expressions (1.36) and (1.34) with

$$
\begin{aligned}
& \mathbf{E}_{1,1}=\mathbf{E}_{2,2}=\mathbf{E}_{3,3}=\mathbf{E}, \\
& \mathbf{E}_{1,2}=\mathbf{E}_{1,3}=\mathbf{E}_{2,1}=\mathbf{E}_{2,3}=\mathbf{E}_{3,1}=\mathbf{E}_{3,2}=\mathbf{0}, \\
& \mathbf{M}_{1,1}=\mathbf{M}_{2,2}=\mathbf{M}_{3,3}=\mathbf{I}, \\
& \mathbf{M}_{1,2}=\mathbf{M}_{1,3}=\mathbf{M}_{2,1}=\mathbf{M}_{2,3}=\mathbf{M}_{3,1}=\mathbf{M}_{3,2}=\mathbf{0} .
\end{aligned}
$$

Consider the transition to the spatial-frequency representation (1.117) using the correct rules of the Fourier expansion of the product of the functions. Derivation of the formulas is presented for the case of an isotropic material. In this case the system of Maxwell's equations (1.26) contains only the following three products: $\varepsilon E_{z}$, $\varepsilon E_{x}, \varepsilon E_{y}$. 
The tangential component $E_{z}$ is continuous, so the product $\varepsilon E_{z}$ is expanded into a Fourier series using the Laurent rules (1.40). In this case, the corresponding matrix $E_{3,3}(1.117)$ has the form (1.121).

We assume that the boundary between media with different dielectric constants in each layer is parallel to the axes [6]. Consider the product $D_{x}=\varepsilon E_{x}$. The product $D_{x}$ is continuous at the sections of the boundaries between two media, parallel to the axis $O y$. Indeed, in these areas $D_{x}=\varepsilon E_{x}$ is a normal component of the electric displacement. The component $E_{x}$ and the function of the dielectric constant $\varepsilon$ are discontinuous at these borders. Thus, the product $D_{x}=$ $\varepsilon E_{x}$ is continuous with respect to $x$ for any fixed $y$.

Accordingly, for the expansion of $D_{x}=\varepsilon E_{x}$ into the Fourier series in the variable $x$ we use the inverse Laurent rule (1.41):

$$
d_{x, i}(y, z)=\sum_{n} \varepsilon_{x i, n}(y) S_{x, n}(y, z),
$$

where $d_{x, i}(y, z), S_{x, n}(y, z)$ are the Fourier coefficients of the functions $D_{x}=\varepsilon E_{x}$, and $\varepsilon_{x i, n}(y)$ are the elements of the Toeplitz matrix $\llbracket 1 / \varepsilon_{x} \rrbracket^{-1}$ formed from the Fourier coefficients with respect to the variable $x$ of the function $1 / \varepsilon(x, y)$. In the sections of the border between the media parallel to the axis $O x$, component $E_{x}$ is tangential and therefore continuous in the variable $y$. The Fourier coefficients $S_{x, n}(y, z)$ of the function $E_{x}$ will also be continuous. Therefore, the expansion in (1.124) of the terms $\varepsilon_{x i, n}(y) S_{x, n}(y, z)$ into the Fourier series in the variable $y$ is performed using the Laurent rule (1.40):

$$
d_{x, i, j}(z)=\sum_{n, m} \varepsilon_{x i, n, j-m} S_{x, n, m}(z),
$$

where $\varepsilon_{x i, n, j-m}$ is the Fourier coefficient with the number $(j-m)$ of the function $\varepsilon_{x i, n}(y)$.

Repeating similar reasoning for $D_{y}=\varepsilon E_{y}$, we obtain

$$
\begin{aligned}
d_{y, j}(x, z) & =\sum_{m} \varepsilon_{y j, m}(x) S_{y, m}(x, z), \\
d_{y, i, j}(z) & =\sum_{n, m} \varepsilon_{y i-n, j, m} S_{y, n, m}(z),
\end{aligned}
$$

where $\varepsilon_{y j, m}(x)$ are the elements of the Toeplitz matrix $\llbracket 1 / \varepsilon_{y} \|^{-1}$ formed from the Fourier coefficients in the variable $y$ of the functions $1 / \varepsilon(x, y)$, and $\varepsilon_{y i-n, j, m}$ is the Fourier coefficient with the number $(i-n)$ of the function $\varepsilon_{y j, m}$. 
The equations (1.125) and (1.127) were obtained with the use of correct rules of expansion into a Fourier series of the products $D_{x}=\varepsilon E_{x}, D_{y}=\varepsilon E_{y}$. Accordingly, in transition from the system (1.26) to the spatial-frequency representation (1.117), the matrices $\mathbf{E}_{1,1}, \mathbf{E}_{2,2}$ will have the form

$$
\begin{aligned}
& E_{1,1 l(i, n), l(j, m)}=\varepsilon_{x i, n, j-m}, \\
& E_{2,2 l(i, n), l(j, m)}=\varepsilon_{y i-n, j, m},
\end{aligned}
$$

where $l(i, j)$ is defined in (1.118), $-N_{x} \leq i, n \leq N_{x},-N_{y} \leq j, m \leq N_{y}$. The matrices (1.128) have the dimensions $\left(2 N_{x}+1\right)\left(2 N_{y}+1\right) \times\left(2 N_{x}\right.$ $+1)\left(2 N_{y}+1\right)$.

As a result, the matrix $\mathbf{A}$ of the system of of the linear differential equations takes the form:

$$
\mathbf{A}=-\left[\begin{array}{cccc}
\mathbf{0} & \mathbf{0} & \mathbf{K}_{y} \mathbf{E}^{-1} \mathbf{K}_{x} & \mathbf{I}-\mathbf{K}_{y} \mathbf{E}^{-1} \mathbf{K}_{y} \\
\mathbf{0} & \mathbf{0} & \mathbf{K}_{x} \mathbf{E}^{-1} \mathbf{K}_{x}-\mathbf{I} & -\mathbf{K}_{x} \mathbf{E}^{-1} \mathbf{K}_{y} \\
\mathbf{K}_{y} \mathbf{K}_{x} & \mathbf{E}_{1,1}-\mathbf{K}_{y}^{2} & \mathbf{0} & \mathbf{0} \\
\mathbf{K}_{x}^{2}-\mathbf{E}_{2,2} & -\mathbf{K}_{x} \mathbf{K}_{y} & \mathbf{0} & \mathbf{0}
\end{array}\right]
$$

A detailed description of the correct rules of expansion into the Fourier series for the general case of the tensors of permittivity and permeability can be found in $[8,9]$.

Subsequent operations of the 'stitching' of the solutions at the layer boundaries and the numerically stable implementation of calculation of the matrix of the system of linear equations for the amplitudes of the diffraction orders are also the same. The matrices $\mathbf{E}, \mathbf{F}$ in the systems of linear equations, representing the tangential field components (1.112), (1.113) at the upper and lower boundaries of the grating, also have the form of (1.74), where

$$
\begin{gathered}
\sin \boldsymbol{\Phi}=\operatorname{diag} \frac{k_{y, j}}{\sqrt{k_{x, i}^{2}+k_{y, j}^{2}}}, \cos \boldsymbol{\Phi}=\operatorname{diag} \frac{k_{x, i}}{\sqrt{k_{x, i}^{2}+k_{y, j}^{2}}}, \\
\cos \boldsymbol{\Phi}^{(\mathrm{U})}=\operatorname{diag}_{l(i, j)} \frac{k_{z, \mathrm{U}, i, j}}{k_{0} n_{\mathrm{U}}}, \cos \boldsymbol{\Phi}^{(\mathrm{D})}=\operatorname{diag} \frac{-k_{z, \mathrm{D}, i, j}}{k_{0} n_{\mathrm{D}}} .
\end{gathered}
$$

Here $\operatorname{diag} f(i, j)$ denotes a diagonal matrix composed of elements $f(i, j)$ arranged in the ascending order of the magnitude of $l(i, j)$. 


\subsubsection{The Fourier modal method for two-dimensional non-periodic structures}

\subsubsection{The geometry of the structure and formulation of the problem}

The Fourier modal method can be adapted to simulate the diffraction of the waveguide and plasmonic modes at inhomogeneities of the waveguide. Consider the method for the case when the geometry of the considered structure is independent of the coordinate $y$. In addition, we assume that, as before, the structure can be divided into regions and in each region $z_{l}<z<z_{l-1}$ the material parameters depend only on the coordinate $x$ (Fig. 1.5). To apply the Fourier modal method we introduce the artificial periodization along the coordinate $x$, while for the elimination of the interaction between adjacent periods (i.e., to ensure vanishing of the electromagnetic field on the borders of the period) special absorbing layers are added to the boundaries of the period. These layers can be represented by multi-layered gradient absorbers or perfectly matched absorbing layers (PML) can be used [21, 22]. The gradient absorbing layers are layers in which the real part of the permittivity equals the permittivity of the adjacent medium, while the imaginary part, which characterizes absorption, increases while approaching the boundary of the period. A disadvantage of the gradient absorbing layers is non-zero reflection of incident radiation on them which increases substantially with increasing incidence angle. In some cases, this fact can substantially affect the solution of the problem with artificial periodization, decreasing the accuracy of the solution with respect to the solution of the original non-periodic problem. Reflections at the boundaries with absorbing layers are eliminated using perfectly matched absorbing layers described in the next section.
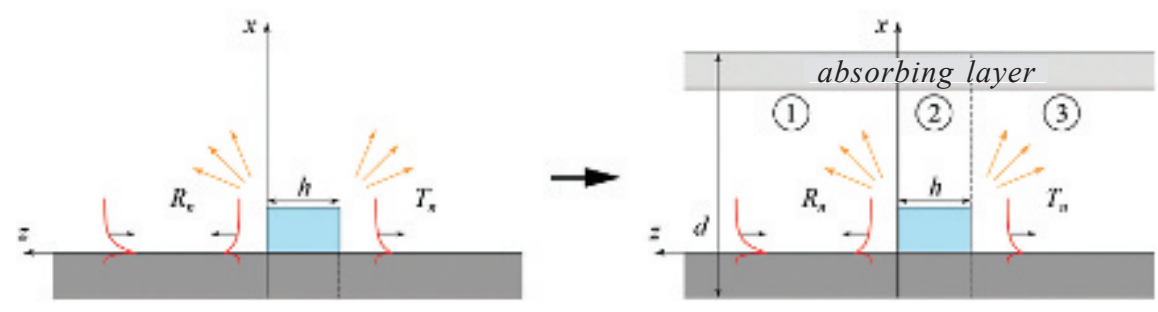

Fig. 1.5. The geometry of the problem of diffraction of surface plasmon polaritons in the aperiodic structure. 
Unlike the standard version of the Fourier modal method, intended for the solution of the problem of diffraction on periodic structures, the result of the application of non-periodic modification will not be a set of intensities of diffraction orders but a set of the amplitudes of the reflected and transmitted modes.

\subsubsection{Perfectly matched absorbing layers}

\section{Perfectly matched absorbing layers as anisotropic materials}

Consider perfectly matched absorbing layers represented by a layer of anisotropic materials added to the boundaries of the period of the structure. The property of the perfectly matched absorbing layers to absorb incident radiation without back-reflection in this case is achieved by a special choice of the permittivity and permeability tensors.

To determine the type of tensor we consider initially an overview of the field of a plane electromagnetic waves in an anisotropic medium, described by the diagonal tensors of dielectric permittivity and magnetic permeability:

$$
[\varepsilon]=\left(\begin{array}{ccc}
\varepsilon_{x} & 0 & 0 \\
0 & \varepsilon_{y} & 0 \\
0 & 0 & \varepsilon_{z}
\end{array}\right),[\mu]=\left(\begin{array}{ccc}
\mu_{x} & 0 & 0 \\
0 & \mu_{y} & 0 \\
0 & 0 & \mu_{z}
\end{array}\right) .
$$

Since the properties of the medium do not depend on the variable $z$, the electric and magnetic fields have the form

$$
\begin{aligned}
& \mathbf{E}(x, y, z)=\mathbf{E}(x, y) \exp \left(i k_{0} \gamma z\right), \\
& \mathbf{H}(x, y, z)=\mathbf{H}(x, y) \exp \left(i k_{0} \gamma z\right) .
\end{aligned}
$$

Substituting (1.131) into (1.3), we obtain:

$$
\begin{array}{cc}
\partial_{y} H_{z}-i k_{0} \gamma H_{y}=-i k_{0} \varepsilon_{x} E_{x}, & \partial_{y} E_{z}-i k_{0} \gamma E_{y}=i k_{0} \mu_{x} H_{x}, \\
-\partial_{x} H_{z}+i k_{0} \gamma H_{x}=-i k_{0} \varepsilon_{y} E_{y}, & -\partial_{x} E_{z}+i k_{0} \gamma E_{x}=i k_{0} \mu_{y} H_{y}, \\
\partial_{x} H_{y}-\partial_{y} H_{x}=-i k_{0} \varepsilon_{z} E_{z}, & \partial_{x} E_{y}-\partial_{y} E_{x}=i k_{0} \mu_{z} H_{z},
\end{array}
$$

where $\partial_{x} f=\partial f / \partial x$. Using (1.133), we represent the tangential components by the components $E_{z}, H_{z}$ in the form 


$$
\begin{aligned}
& E_{x}=\frac{-1}{i k_{0}\left(\varepsilon_{x}-\gamma^{2} / \mu_{y}\right)}\left(\partial_{y} H_{z}+\frac{\gamma}{\mu_{y}} \cdot \partial_{x} E_{z}\right), \\
& E_{y}=\frac{1}{i k_{0}\left(\varepsilon_{y}-\gamma^{2} / \mu_{x}\right)}\left(\partial_{x} H_{z}-\frac{\gamma}{\mu_{x}} \cdot \partial_{y} E_{z}\right), \\
& H_{x}=\frac{1}{i k_{0}\left(\mu_{x}-\gamma^{2} / \varepsilon_{y}\right)}\left(\partial_{y} E_{z}-\frac{\gamma}{\varepsilon_{y}} \cdot \partial_{x} H_{z}\right), \\
& H_{y}=\frac{-1}{i k_{0}\left(\mu_{y}-\gamma^{2} / \varepsilon_{x}\right)}\left(\partial_{x} E_{z}+\frac{\gamma}{\varepsilon_{x}} \cdot \partial_{y} H_{z}\right),
\end{aligned}
$$

where $E_{z}, H_{z}$ satisfy the equations

$$
\begin{aligned}
& \frac{1}{\left(\mu_{y}-\gamma^{2} / \varepsilon_{x}\right)}\left(\frac{\partial^{2} E_{z}}{\partial x^{2}}+\frac{\gamma}{\varepsilon_{x}} \cdot \frac{\partial^{2} H_{z}}{\partial x \partial y}\right)+ \\
& +\frac{1}{\left(\mu_{x}-\gamma^{2} / \varepsilon_{y}\right)}\left(\frac{\partial^{2} E_{z}}{\partial y^{2}}-\frac{\gamma}{\varepsilon_{y}} \cdot \frac{\partial^{2} H_{z}}{\partial y \partial x}\right)=-k_{0}^{2} \varepsilon_{z} E_{z}, \\
& \frac{1}{\left(\varepsilon_{y}-\gamma^{2} / \mu_{x}\right)}\left(\frac{\partial^{2} H_{z}}{\partial x^{2}}-\frac{\gamma}{\mu_{x}} \cdot \frac{\partial^{2} E_{z}}{\partial x \partial y}\right)+ \\
& +\frac{1}{\left(\varepsilon_{x}-\gamma^{2} / \mu_{y}\right)}\left(\frac{\partial^{2} H_{z}}{\partial y^{2}}+\frac{\gamma}{\mu_{y}} \cdot \frac{\partial^{2} E_{z}}{\partial y \partial x}\right)=-k_{0}^{2} \mu_{z} H_{z} .
\end{aligned}
$$

For an uniaxial anisotropic medium of the type

$$
[\varepsilon]=\left(\begin{array}{ccc}
\varepsilon & 0 & 0 \\
0 & \varepsilon & 0 \\
0 & 0 & \varepsilon_{z}
\end{array}\right),[\mu]=\left(\begin{array}{ccc}
\mu & 0 & 0 \\
0 & \mu & 0 \\
0 & 0 & \mu_{z}
\end{array}\right)
$$

we obtain from (1.135) that $E_{z}, H_{z}$ satisfy the Helmholtz equation

$$
\begin{aligned}
& \frac{\partial^{2} E_{z}}{\partial x^{2}}+\frac{\partial^{2} E_{z}}{\partial y^{2}}=-k_{0}^{2} \varepsilon_{z}\left(\mu-\gamma^{2} / \varepsilon\right) E_{z}, \\
& \frac{\partial^{2} H_{z}}{\partial x^{2}}+\frac{\partial^{2} H_{z}}{\partial y^{2}}=-k_{0}^{2} \mu_{z}\left(\varepsilon-\gamma^{2} / \mu\right) H_{z} .
\end{aligned}
$$


Solving (1.137) by separation of variables, we obtain

$$
\begin{aligned}
& E_{z}(x, y, z)=\exp \left(i k_{0} \sqrt{\varepsilon_{z} \mu}(\alpha x+\beta y) \pm i k_{0} \gamma z\right), \\
& H_{z}(x, y, z)=\exp \left(i k_{0} \sqrt{\varepsilon_{z} \mu}(\alpha x+\beta y) \pm i k_{0} \gamma_{1} z\right),
\end{aligned}
$$

where

$$
\gamma=\sqrt{\varepsilon \mu} \sqrt{1-\alpha^{2}-\beta^{2}}, \gamma_{1}=\mu \sqrt{\varepsilon / \mu-\left(\alpha^{2}+\beta^{2}\right) \varepsilon_{z} / \mu_{z}} .
$$

Next, we consider the E- and H-type waves. For the E-wave $E_{z} \neq 0, H_{z}=0$, and for the H-wave $H_{z} \neq 0, E_{z}=0$. From (1.134) (1.138) for the E-waves we get

$$
\begin{aligned}
& E_{x}=\frac{\mp \gamma}{i k_{0}\left(\varepsilon \mu-\gamma^{2}\right)} \cdot \partial_{x} E_{z}=\frac{\mp \alpha \gamma \sqrt{\varepsilon_{z} \mu}}{\left(\mu \varepsilon-\gamma^{2}\right)} \cdot \exp \left(i k_{0} \sqrt{\varepsilon_{z} \mu}(\alpha x+\beta y) \pm i k_{0} \gamma z\right), \\
& E_{y}=\frac{\mp \gamma}{i k_{0}\left(\varepsilon \mu-\gamma^{2}\right)} \cdot \partial_{y} E_{z}=\frac{\mp \gamma \beta \sqrt{\varepsilon_{z} \mu}}{\left(\varepsilon \mu-\gamma^{2}\right)} \cdot \exp \left(i k_{0} \sqrt{\varepsilon_{z} \mu}(\alpha x+\beta y) \pm i k_{0} \gamma z\right), \\
& H_{x}=\frac{\varepsilon}{i k_{0}\left(\mu \varepsilon-\gamma^{2}\right)} \partial_{y} E_{z}=\frac{\varepsilon \beta \sqrt{\varepsilon_{z} \mu}}{\left(\mu \varepsilon-\gamma^{2}\right)} \cdot \exp \left(i k_{0} \sqrt{\varepsilon_{z} \mu}(\alpha x+\beta y) \pm i k_{0} \gamma z\right), \\
& H_{y}=\frac{-\varepsilon}{i k_{0}\left(\mu \varepsilon-\gamma^{2}\right)} \partial_{x} E_{z}=\frac{-\varepsilon \alpha \sqrt{\varepsilon_{z} \mu}}{\left(\mu \varepsilon-\gamma^{2}\right)} \cdot \exp \left(i k_{0} \sqrt{\varepsilon_{z} \mu}(\alpha x+\beta y) \pm i k_{0} \gamma z\right) .
\end{aligned}
$$

Since the solution of the diffraction problem requires the imposition of conditions of equality of the tangential components of the fields at the interface, it is convenient to introduce the following four-component vector of the tangential components:

$$
\mathbf{E}_{E}=\left(\begin{array}{c}
E_{x} \\
H_{y} \\
E_{y} \\
H_{x}
\end{array}\right)=\frac{1}{\sqrt{\gamma \varepsilon\left(\alpha^{2}+\beta^{2}\right)}}\left(\begin{array}{c}
\mp \alpha \gamma \\
-\varepsilon \alpha \\
\mp \gamma \beta \\
\varepsilon \beta
\end{array}\right) \exp \left(i k_{0} \sqrt{\varepsilon_{z} \mu}(\alpha x+\beta y) \pm i k_{0} \gamma z\right)
$$

Vector (1.141) is written with a normalizing factor chosen from the condition that the unit $z$-component of the Poynting vector $S_{z}=\left|E_{x} H_{y}-E_{y} H_{x}\right|$ is unity. 
From (1.134) and (1.138) for the H-wave we get

$$
\begin{aligned}
& E_{x}=\frac{-\mu}{i k_{0}\left(\varepsilon \mu-\gamma_{1}^{2}\right)} \partial_{y} H_{z}=\frac{-\mu \beta \sqrt{\varepsilon_{z} \mu}}{\left(\varepsilon \mu-\gamma_{1}^{2}\right)} \cdot \exp \left(i k_{0} \sqrt{\varepsilon_{z} \mu}(\alpha x+\beta y) \pm i k_{0} \gamma_{1} z\right), \\
& H_{y}=\frac{\mp \gamma_{1}}{i k_{0}\left(\mu-\gamma_{1}^{2}\right)} \cdot \partial_{y} H_{z}=\frac{\mp \gamma_{1} \beta \sqrt{\varepsilon_{z} \mu}}{\left(\mu-\gamma_{1}^{2}\right)} \cdot \exp \left(i k_{0} \sqrt{\varepsilon_{z} \mu}(\alpha x+\beta y) \pm i k_{0} \gamma_{1} z\right), \\
& E_{y}=\frac{\mu}{i k_{0}\left(\varepsilon \mu-\gamma_{1}^{2}\right)} \cdot \partial_{x} H_{z}=\frac{\mu \alpha \sqrt{\varepsilon_{z} \mu}}{\left(\varepsilon \mu-\gamma_{1}^{2}\right)} \cdot \exp \left(i k_{0} \sqrt{\varepsilon_{z} \mu}(\alpha x+\beta y) \pm i k_{0} \gamma_{1} z\right), \\
& H_{x}=\frac{\mp \gamma_{1}}{i k_{0}\left(\mu \varepsilon-\gamma_{1}^{2}\right)} \cdot \partial_{x} H_{z}=\frac{\mp \gamma_{1} \alpha \sqrt{\varepsilon_{z} \mu}}{\left(\mu \varepsilon-\gamma_{1}^{2}\right)} \cdot \exp \left(i k_{0} \sqrt{\varepsilon_{z} \mu}(\alpha x+\beta y) \pm i k_{0} \gamma_{1} z\right) .
\end{aligned}
$$

Similarly to (1.141), we introduce the vector of the tangential components of the $\mathrm{H}$-wave in the form

$$
\mathbf{E}_{H}=\left(\begin{array}{c}
E_{x} \\
H_{y} \\
E_{y} \\
H_{x}
\end{array}\right)=\frac{1}{\sqrt{\gamma_{1} \mu\left(\alpha^{2}+\beta^{2}\right)}}\left(\begin{array}{c}
-\mu \beta \\
\mp \gamma_{1} \beta \\
\mu \alpha \\
\mp \gamma_{1} \alpha
\end{array}\right) \exp \left(i k_{0} \sqrt{\varepsilon_{z} \mu}(\alpha x+\beta y) \pm i k_{0} \gamma_{1} z\right) .
$$

Fresnel equations. We write the Fresnel equations for the interface between a homogeneous dielectric and a medium described by the tensor (1.136). Without the loss of generality, we assume that the interface is the plane $z=0$. We also assume that in the region $z>0$ there is a homogeneous dielectric, and at $z<0-$ the anisotropic medium (1.136).

Suppose that a plane wave impinges on the interface from the side of the homogeneous dielectric. The plane wave is represented as a superposition of the E- and H-waves. In a general case, upon the reflection and refraction of the incident wave at the interface reflected and refracted waves will be formed as a superposition of the E- and H-waves.

Assuming in (1.141) and (1.143) $\varepsilon=\varepsilon_{z}=\varepsilon_{0}, \mu=\mu_{z}=\mu_{0}$, we write the $\mathrm{E}-$ and $\mathrm{H}$-waves incident and reflected from the interface 


$$
\begin{aligned}
& \mathbf{E}_{i n c, E}=\frac{1}{\sqrt{\gamma_{0} \varepsilon_{0}\left(\alpha_{0}^{2}+\beta_{0}^{2}\right)}}\left(\begin{array}{c}
\alpha_{0} \gamma_{0} \\
-\varepsilon_{0} \alpha_{0} \\
\gamma_{0} \beta_{0} \\
\varepsilon_{0} \beta_{0}
\end{array}\right) \exp \left(i k_{0} \sqrt{\varepsilon_{0} \mu_{0}}\left(\alpha_{0} x+\beta_{0} y\right)-i k_{0} \gamma_{0} z\right), \\
& \mathbf{E}_{\text {inc }, H}=\frac{1}{\sqrt{\gamma_{0} \mu_{0}\left(\alpha_{0}^{2}+\beta_{0}^{2}\right)}}\left(\begin{array}{c}
-\mu_{0} \beta_{0} \\
\gamma_{0} \beta_{0} \\
\mu_{0} \alpha_{0} \\
\gamma_{0} \alpha_{0}
\end{array}\right) \exp \left(i k_{0} \sqrt{\varepsilon_{0} \mu_{0}}\left(\alpha_{0} x+\beta_{0} y\right)-i k_{0} \gamma_{0} z\right) \text {, } \\
& \mathbf{E}_{r e f, E}=\frac{1}{\sqrt{\gamma_{0} \varepsilon_{0}\left(\alpha_{0}^{2}+\beta_{0}^{2}\right)}}\left(\begin{array}{c}
-\alpha_{0} \gamma_{0} \\
-\varepsilon_{0} \alpha_{0} \\
-\gamma_{0} \beta_{0} \\
\varepsilon_{0} \beta_{0}
\end{array}\right) \exp \left(i k_{0} \sqrt{\varepsilon_{0} \mu_{0}}\left(\alpha_{0} x+\beta_{0} y\right)+i k_{0} \gamma_{0} z\right), \\
& \mathbf{E}_{r e f, H}=\frac{1}{\sqrt{\gamma_{0} \mu_{0}\left(\alpha_{0}^{2}+\beta_{0}^{2}\right)}}\left(\begin{array}{c}
-\mu_{0} \beta_{0} \\
-\gamma_{0} \beta_{0} \\
\mu_{0} \alpha_{0} \\
-\gamma_{0} \alpha_{0}
\end{array}\right) \exp \left(i k_{0} \sqrt{\varepsilon_{0} \mu_{0}}\left(\alpha_{0} x+\beta_{0} y\right)+i k_{0} \gamma_{0} z\right) \text {, }
\end{aligned}
$$

where $\gamma_{0}=\sqrt{\varepsilon_{0} \mu_{0}} \sqrt{1-\alpha_{0}^{2}-\beta_{0}^{2}}$. 'inc' and 'ref' in the subscripts of the waves in (1.144) and (1.145) denote the incident and reflected waves, respectively.

If $z>0$ the field is a superposition of the incident and reflected waves

$$
\mathbf{E}_{z>0}(\mathbf{x})=I_{E} \mathbf{E}_{i n c, E}(\mathbf{x})+I_{H} \mathbf{E}_{i n c, H}(\mathbf{x})+R_{E} \mathbf{E}_{r e f, E}(\mathbf{x})+R_{H} \mathbf{E}_{r e f, H}(\mathbf{x}),
$$

where $\mathbf{x}=(x, y, z), I_{E}, I_{H}$ are the coefficients of the E- and H-waves for the incident wave, and $R_{E}, R_{H}$ are the reflection coefficients. The transmitted field $z<0$ has the form:

$$
\mathbf{E}_{z<0}(\mathbf{x})=T_{E} \cdot \mathbf{E}_{t r, E}(\mathbf{x})+T_{H} \cdot \mathbf{E}_{t r, H}(\mathbf{x})
$$

where $T_{E}, T_{H}$ are the transmission coefficients, 


$$
\begin{aligned}
& \mathbf{E}_{t r, E}=\frac{1}{\sqrt{\gamma \varepsilon\left(\alpha^{2}+\beta^{2}\right)}}\left(\begin{array}{c}
\alpha \gamma \\
-\varepsilon \alpha \\
\gamma \beta \\
\varepsilon \beta
\end{array}\right) \exp \left(i k_{0} \sqrt{\varepsilon_{z} \mu}(\alpha x+\beta y)-i k_{0} \gamma z\right), \\
& \mathbf{E}_{t r, H}=\frac{1}{\sqrt{\gamma_{1} \mu\left(\alpha^{2}+\beta^{2}\right)}}\left(\begin{array}{c}
-\mu \beta \\
\gamma_{1} \beta \\
\mu \alpha \\
\gamma_{1} \alpha
\end{array}\right) \exp \left(i k_{0} \sqrt{\varepsilon_{z} \mu}(\alpha x+\beta y)-i k_{0} \gamma_{1} z\right),
\end{aligned}
$$

where $\gamma=\sqrt{\varepsilon \mu} \sqrt{1-\alpha^{2}-\beta^{2}}, \gamma_{1}=\mu \sqrt{\varepsilon / \mu-\left(\alpha^{2}+\beta^{2}\right) \varepsilon_{z} / \mu_{z}}$.

To determine the reflection and transmission coefficients, we write the condition of equality of the tangential components at $z=0$ $\left(\mu_{0}=1\right)$ :

$$
\begin{gathered}
\frac{I_{E}}{\sqrt{\gamma_{0} \varepsilon_{0}\left(\alpha_{0}^{2}+\beta_{0}^{2}\right)}}\left(\begin{array}{c}
\gamma_{0} \alpha_{0} \\
-\varepsilon_{0} \alpha_{0} \\
\gamma_{0} \beta_{0} \\
\varepsilon_{0} \beta_{0}
\end{array}\right)+\frac{I_{H}}{\sqrt{\gamma_{0}\left(\alpha_{0}^{2}+\beta_{0}^{2}\right)}}\left(\begin{array}{c}
-\beta_{0} \\
\gamma_{0} \beta_{0} \\
\alpha_{0} \\
\gamma_{0} \alpha_{0}
\end{array}\right)+\frac{R_{E}}{\sqrt{\gamma_{0} \varepsilon_{0}\left(\alpha_{0}^{2}+\beta_{0}^{2}\right)}}\left(\begin{array}{c}
-\gamma_{0} \alpha_{0} \\
-\varepsilon_{0} \alpha_{0} \\
-\gamma_{0} \beta_{0} \\
\varepsilon_{0} \beta_{0}
\end{array}\right)+ \\
+\frac{R_{H}}{\sqrt{\gamma_{0}\left(\alpha_{0}^{2}+\beta_{0}^{2}\right)}}\left(\begin{array}{c}
\alpha \gamma \\
-\gamma_{0} \beta_{0} \\
\alpha_{0} \\
-\gamma_{0} \alpha_{0}
\end{array}\right)=\frac{T_{E}}{\sqrt{\gamma \varepsilon\left(\alpha^{2}+\beta^{2}\right)}}\left(\begin{array}{c}
-\mu \beta \\
-\varepsilon \alpha \\
\gamma \beta \\
\gamma_{1} \beta \\
\mu \alpha \\
\gamma_{1} \alpha
\end{array}\right)+\frac{T_{H}}{\sqrt{\gamma_{1} \mu\left(\alpha^{2}+\beta^{2}\right)}} .
\end{gathered}
$$

The refraction law should be satisfied in this case;

$$
\alpha_{0} \sqrt{\varepsilon_{0}}=\alpha \sqrt{\varepsilon_{z} \mu}, \quad \beta_{0} \sqrt{\varepsilon_{0}}=\beta \sqrt{\varepsilon_{z} \mu}
$$

In the particular case

$$
[\varepsilon]=\varepsilon_{0}\left(\begin{array}{ccc}
a & 0 & 0 \\
0 & a & 0 \\
0 & 0 & 1 / a
\end{array}\right),[\mu]=\left(\begin{array}{ccc}
a & 0 & 0 \\
0 & a & 0 \\
0 & 0 & 1 / a
\end{array}\right)
$$


we obtain

$$
\alpha=\alpha_{0}, \beta=\beta_{0}, \gamma=\gamma_{1}=a \sqrt{\varepsilon_{0}} \sqrt{1-\alpha_{0}^{2}-\beta_{0}^{2}}=a \gamma_{0} .
$$

In this case, the equation (1.149) takes the form

$$
\begin{gathered}
\frac{I_{E}}{\sqrt{\gamma_{0} \varepsilon_{0}\left(\alpha_{0}^{2}+\beta_{0}^{2}\right)}}\left(\begin{array}{c}
\gamma_{0} \alpha_{0} \\
-\varepsilon_{0} \alpha_{0} \\
\gamma_{0} \beta_{0} \\
\varepsilon_{0} \beta_{0}
\end{array}\right)+\frac{I_{H}}{\sqrt{\gamma_{0}\left(\alpha_{0}^{2}+\beta_{0}^{2}\right)}}\left(\begin{array}{c}
-\beta_{0} \\
\gamma_{0} \beta_{0} \\
\alpha_{0} \\
\gamma_{0} \alpha_{0}
\end{array}\right)+\frac{R_{E}}{\sqrt{\gamma_{0} \varepsilon_{0}\left(\alpha_{0}^{2}+\beta_{0}^{2}\right)}}\left(\begin{array}{c}
-\gamma_{0} \alpha_{0} \\
-\varepsilon_{0} \alpha_{0} \\
-\gamma_{0} \beta_{0} \\
\varepsilon_{0} \beta_{0}
\end{array}\right)+ \\
+\frac{R_{H}}{\sqrt{\gamma_{0}\left(\alpha_{0}^{2}+\beta_{0}^{2}\right)}}\left(\begin{array}{c}
\alpha_{0} \gamma_{0} \\
-\gamma_{0} \beta_{0} \\
\alpha_{0} \\
-\varepsilon_{0} \alpha_{0} \\
\gamma_{0} \beta_{0} \\
\varepsilon_{0} \beta_{0}
\end{array}\right)+\frac{T_{E}}{\sqrt{\gamma_{0} \varepsilon_{0}\left(\alpha_{0}^{2}+\beta_{0}\left(\alpha_{0}^{2}+\beta_{0}^{2}\right)\right.}}\left(\begin{array}{c}
-\beta_{0} \\
\gamma_{0} \beta_{0} \\
\alpha_{0} \\
\gamma_{0} \alpha_{0}
\end{array}\right) .
\end{gathered}
$$

It is easy to see that the solution of (1.153) is as follows:

$$
R_{E}=R_{H}=0, T_{H}=I_{H}, T_{E}=I_{E} .
$$

According to (1.154), medium (1.151) does not reflect the incident waves. At complex $a=a^{\prime}+i a^{\prime \prime}$ in (1.151) the transmitted waves (1.148) will be evansecent. The rate of decay is determined by the imaginary part $a^{\prime \prime}$. Thus, a layer of material (1.151) at complex $a$ is a perfectly matched absorbing layer.

When $\beta_{0}=0$ in (1.144) the direction vector of the incident wave lies in the plane $x O z$. In the case of 'planar incidence' the E-wave corresponds to a wave with TM-polarization and the H-wave to a wave with TE-polarization.

For the incident wave with TM-polarization the medium (1.151) takes the form

$$
[\varepsilon]=\varepsilon_{0}\left(\begin{array}{ccc}
a & 0 & 0 \\
0 & a & 0 \\
0 & 0 & 1 / a
\end{array}\right), \mu=a
$$

Indeed, it is easy to show that under the condition (1.155), $\beta_{0}=$ 0 and $I_{H}=0$ the solution of (1.149) has the form 


$$
R_{E}=R_{H}=0, T_{H}=0, T_{E}=I_{E}
$$

According to (1.156), the medium (1.155) does not reflect the incident waves.

Similarly, for the incident wave with TE-polarization the following condition is sufficient

$$
\varepsilon=\varepsilon_{0} a,[\mu]=\left(\begin{array}{ccc}
a & 0 & 0 \\
0 & a & 0 \\
0 & 0 & 1 / a
\end{array}\right)
$$

In the case of (1.157) the solution of (1.149) with $\beta_{0}=0$ and $I_{H}=0$ has the form

$$
R_{E}=R_{H}=0, T_{H}=I_{H}, T_{E}=0 .
$$

\section{Perfectly matched absorbing layers as a complex coordinate transformation}

We now introduce the perfectly matched absorbing layers as a complex coordinate transformation [23]. For simplicity, consider the case of diffraction of a TM-polarized wave (in particular, the plasmonic modes - the geometry of the structure is shown on the left in Fig. 1.6) on the structure composed of isotropic materials. In this case, the electric and magnetic fields have the form $\mathbf{E}=\left(E_{x}, 0, E_{z}\right), \mathbf{H}=\left(0, H_{y}, 0\right)$. In each of the layers of the structure at $\mu=1$ we can obtain the Helmholtz equation for the component $H_{y}$ from Maxwell's equations (1.24):

$$
\frac{\partial^{2} H_{y}}{\partial z^{2}}+\varepsilon(x) \frac{\partial}{\partial x}\left[\frac{1}{\varepsilon(x)} \frac{\partial H_{y}}{\partial x}\right]+k_{0}^{2} \varepsilon(x) H_{y}=0 .
$$

Equation (1.159) is written in a general form and is valid for both the initial non-periodic problem and, for example, for the problem of diffraction of the TM-polarized wave on a diffraction structure with one-dimensional periodicity. Note that in solving the diffraction problem by the Fourier modal method, equation (1.159), which describes the field in the layer of the structure, is reduced to a second system of differential equations in (1.48). 


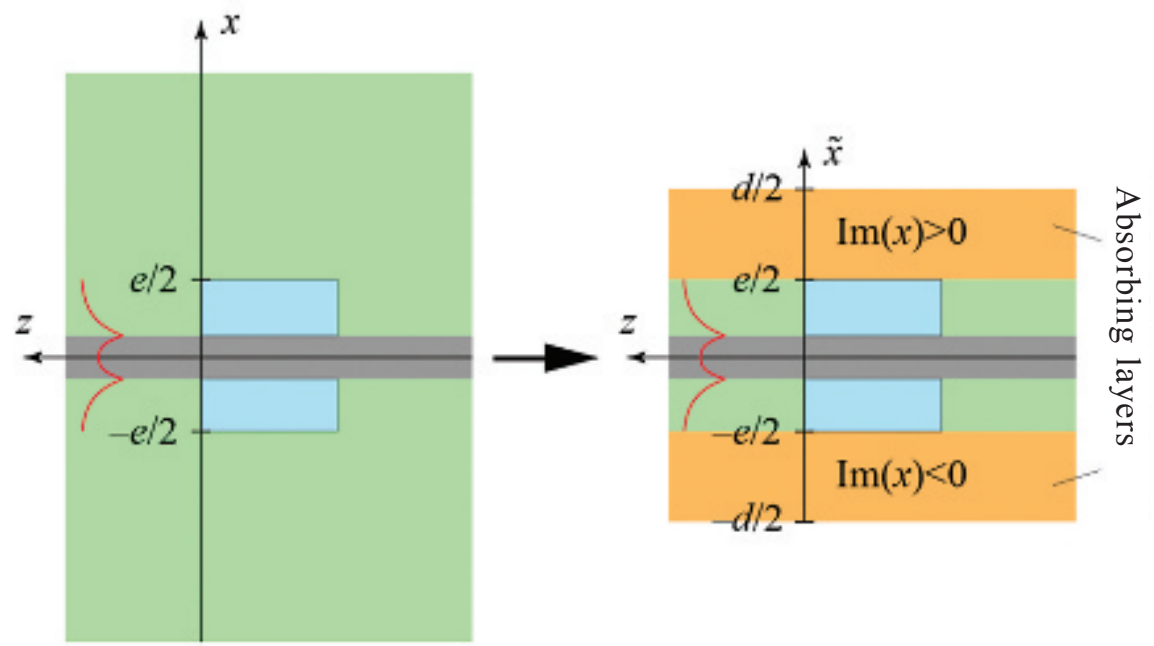

Fig. 1.6. The transition to a non-periodic task using perfectly matched absorbing layers in the form of coordinate transformations.

Suppose we are interested in the solution of the original aperiodic problem in the region $|x|>e / 2$ in addition, the regions $|x|>e / 2$ are homogeneous (Fig. 1.6). Note that because of physical reasons (absence of incident waves apart from the incident waveguide or plasmonic mode) the regions $|x|>e / 2$ contain only the waves propagating from the structure (in the direction of increase $|x|>e / 2$ ).

As indicated above, to solve a non-periodic problem by the Fourier modal method it is necessary to introduce artificial periodization with a certain period $d>e$ so as to eliminate the interaction between adjacent periods. To do this, it is necessary to ensure that the components of the electromagnetic field at the boundaries between periods are equal to zero. Consider the analytic continuation of the solution of equation (1.159) for the original problem in the variable $x$ in the complex plane: $x=x^{\prime}+i x^{\prime \prime}$. Note that the propagating waves are evanescent in this case. Indeed, for waves propagating in the positive direction of the $x$ axis $\left(k_{x}>0\right)$, we get:

$$
\exp \left(i k_{x} x\right)=\exp \left(i k_{x} x^{\prime}\right) \exp \left(-k_{x} x^{\prime \prime}\right)
$$

Thus, these waves become evanescent when $x^{\prime \prime} \rightarrow+\infty$. Similarly, the waves propagating in the negative direction of the axis $x$, decay when $x^{\prime \prime} \rightarrow-\infty$. Thus, we consider the solution of (1.159) on the contour in the complex plane having the form shown in Fig. 1.7. 
The horizontal dashed line shows the real axis, the vertical dotted lines - the area $x^{\prime} / d \in[-e /(2 d), e /(2 d)]$.

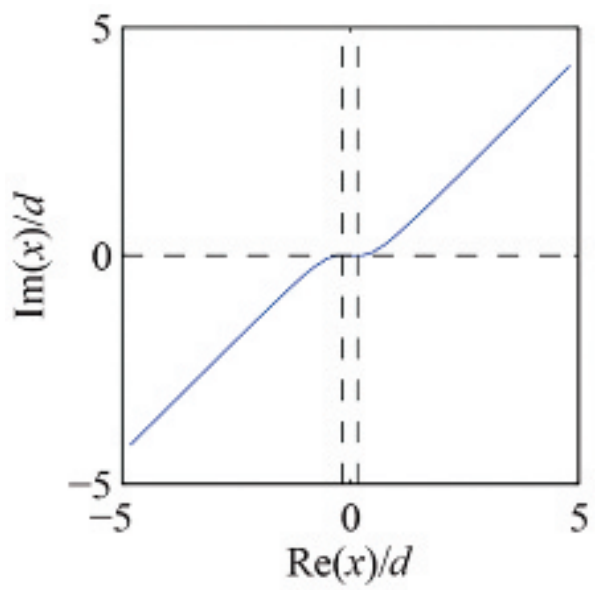

Fig. 1.7. The considered contour in the complex plane.

At $x^{\prime} \in(-e / 2, e / 2)$ the contour lies on the real axis, away from the real axis $x^{\prime \prime} \rightarrow \pm \infty$ and the imaginary part $x^{\prime}$ also tends to infinity, with the condition $x^{\prime} x^{\prime \prime}>0$. This selection of the contour provides decay of the electromagnetic field with increasing distance from the real axis. To introduce artificial periodization, consider the transformation of coordinates $F(\tilde{x})=x$ mapping the contour on the complex plane into the interval $\tilde{x} \in(-d / 2, d / 2)$ where $\tilde{x}$ is a new real coordinate. At the same time at $\tilde{x} \in(-e / 2, e / 2)$ the field will be the same as the solution of the original equation (1.159), while at $\tilde{x} \rightarrow \pm d / 2$ the field components will tend to zero by the choice of the contour in the complex plane $x$. To move from equation (1.159) to a new differential equations depending on a variable $\tilde{x}$, we need to make the following change of the differential operator: $\partial / \partial x \rightarrow f \cdot \partial / \partial \tilde{x}$ where $f(\tilde{x})=d \tilde{x} / d x=(d F(\tilde{x}) / d \tilde{x})^{-1}$. As a result, we obtain the differential equation

$$
\frac{\partial^{2} H_{y}}{\partial z^{2}}+\varepsilon(x) f \frac{\partial}{\partial \tilde{x}}\left[\frac{f}{\varepsilon(x)} \frac{\partial H_{y}}{\partial \tilde{x}}\right]+k_{0}^{2} \varepsilon(x) H_{y}=0 .
$$

Equation (1.161) can be solved by the Fourier modal method since the task now allows artificial periodization (since the field at the boundaries of the period $\tilde{x}= \pm d / 2$ tends to zero). Using the 
expansions (1.27), the solution of the differential equation (1.161), which describes a general representation of the field in the layer can be reduced to a system of differential equations

$$
\frac{d}{d z^{\prime}}\left[\begin{array}{l}
\mathbf{U}_{y} \\
\mathbf{S}_{x}
\end{array}\right]=-\left[\begin{array}{cc}
\mathbf{0} & \mathbf{E}^{*} \\
\mathbf{F}_{x} \mathbf{K}_{x} \mathbf{E}^{-1} \mathbf{F}_{x} \mathbf{K}_{x}-\mathbf{I} & \mathbf{0}
\end{array}\right]\left[\begin{array}{l}
\mathbf{U}_{y} \\
\mathbf{S}_{x}
\end{array}\right],
$$

where $z^{\prime}=k_{0} z, \mathbf{F}_{x}$ is the Toeplitz matrix formed from the coefficients of expansion of the function $f$ into the Fourier series. Comparing the second system of differential equations (1.48) with (1.162), one can see that the latter is derived from the first formal change $\boldsymbol{K}_{x} \rightarrow \boldsymbol{F}_{x} \boldsymbol{K}_{x}$. Note that in the general case, when the field in the layer is described by a system of differential equations (1.35) with the matrix (1.36), the perfectly matched absorbing layers can be introduced using the same formal change.

We now give as an example the type of function $f(\tilde{x})$ that describes the coordinate transformation [23]:

$$
f(\tilde{x})=\left\{\begin{array}{l}
1,|\tilde{x}|<e / 2, \\
{\left[1-\gamma \sin ^{2}\left(\pi \frac{|\tilde{x}|-e / 2}{q}\right)\right] \cos ^{2}\left(\pi \frac{|\tilde{x}|-e / 2}{q}\right), \frac{e}{2}<|\tilde{x}|<\frac{d}{2},}
\end{array}\right.
$$

where $q=d-e$ is the total size of the region corresponding to the absorbing layers, $\gamma$ is a complex parameter characterizing the absorbing layer (typical value $\gamma=1 /(1-i)$ ). For this function, the coefficients of the Fourier series can be found analytically and have the form

$$
\begin{aligned}
& f_{n}=\delta_{n 0}-\frac{q}{2 d}(-1)^{n}\left[\left(1+\frac{\gamma}{4}\right) \operatorname{sinc}\left(\frac{n q}{d}\right)+\frac{1}{2} \operatorname{sinc}\left(\frac{n q}{d}-1\right)+\right. \\
& \left.+\frac{1}{2} \operatorname{sinc}\left(\frac{n q}{d}+1\right)-\frac{\gamma}{8} \operatorname{sinc}\left(\frac{n q}{d}-2\right)-\frac{\gamma}{8} \operatorname{sinc}\left(\frac{n q}{d}+2\right)\right],
\end{aligned}
$$

where $\delta_{n 0}$ is the Kronecker symbol, $\operatorname{sinc}(x)=\sin (\pi x) /(\pi x)$. We note that the function $f(\tilde{x})$ corresponds to the contour in the complex plane 


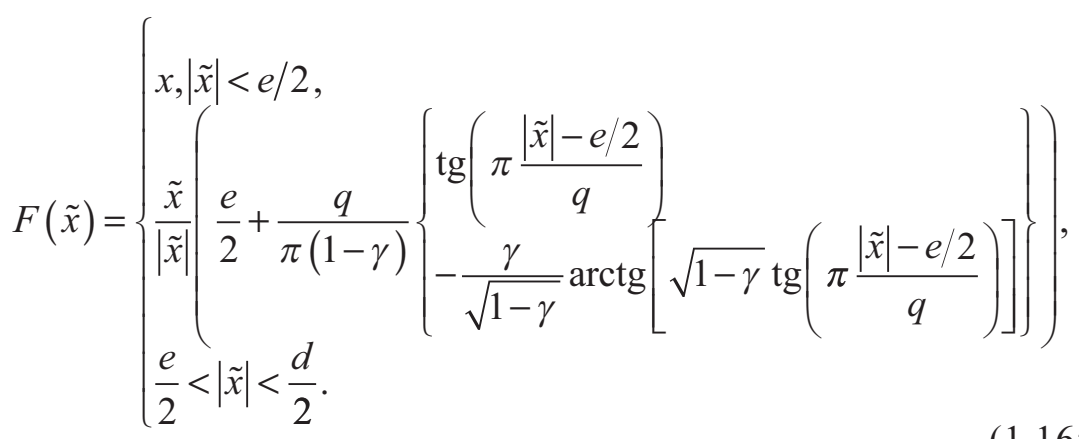

The real and imaginary parts of the function $f(\tilde{x})$ at $e=d / 3, \gamma=$ $1 /(1-i)$ are shown in Fig. 1.8, the contour in the complex plane is shown in Fig. 1.7.

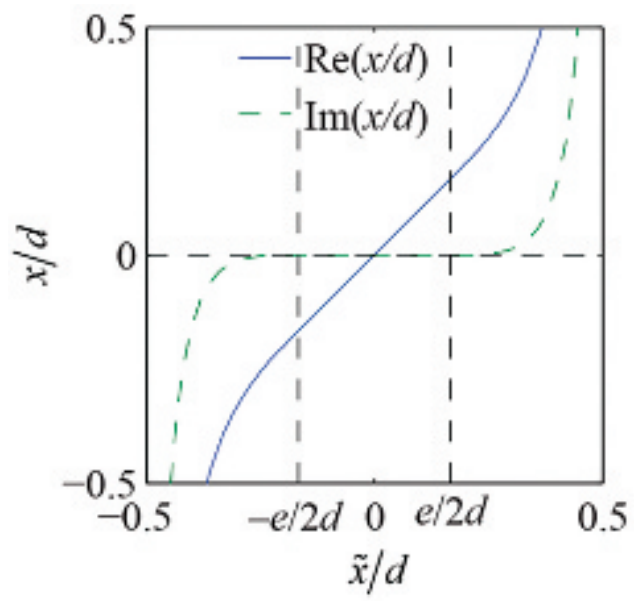

Fig. 1.8. The real and imaginary parts of the contour in the complex plane, depending on the value of the new real variable.

\subsubsection{Solution of the diffraction problem}

Stages of solving the difraction problem in this case coincide with the stages of solving the diffraction problem of a plane wave on a two-dimensional diffraction structure described in subsection 1.2. The differences lie in the form of the field above and below the structure (in regions 1 and 3 in Fig. 1.5). Since the regions above and below the structure in this case are not uniform, the electromagnetic field therein can not be represented in the form of Rayleigh expansions (1.20) and (1.21). Representation of the field in these areas can be obtained similarly to the representation of the field in the layer of 
the structure obtained by solving a system of differential equations of the form (1.35) (with the possible modification of the matrix of the system by introducing perfectly matched layers in the form of coordinate transformations). Thus, the field above and below the structure will be presented in the form of the Fourier series (1.27) and the expressions for the $x$ - and $y$-Fourier components of the fields will be similar to (1.58):

$$
\begin{aligned}
{\left[\begin{array}{c}
\mathbf{S}_{y}^{U} \\
\mathbf{S}_{x}^{U} \\
\mathbf{U}_{y}^{U} \\
\mathbf{U}_{x}^{U}
\end{array}\right]=} & \mathbf{W}_{i n c} \exp \left(\lambda_{i n c} k_{0} z\right) A_{i n c}+\mathbf{W}_{U}^{(-)} \exp \left(\boldsymbol{\Lambda}_{U}^{(-)} k_{0} z\right) \mathbf{R} \\
& {\left[\begin{array}{c}
\mathbf{S}_{y}^{D} \\
\mathbf{S}_{x}^{D} \\
\mathbf{U}_{y}^{D} \\
\mathbf{U}_{x}^{D}
\end{array}\right]=\mathbf{W}_{D}^{(+)} \exp \left[\Lambda_{D}^{(+)} k_{0}\left(z-z_{L}\right)\right] \mathbf{T} . }
\end{aligned}
$$

Similarly to the expressions (1.20), (1.21), the indices $U$ and $D$ correspond to the regions above and below the structure (reas 1 and 3 in Fig. 1.5). The first term in (1.166) describes the incident wave with an amplitude $A_{i n c}$, with its eigenvalue $\lambda_{i n c}$ selected from $\boldsymbol{\Lambda}_{U}^{(+)}$as closest one to the propagation constant of the incident mode calculated by solving the corresponding dispersion equation.

The system of linear equations (1.71), which describes the condition of equality of the tangential components of the electromagnetic field at the boundaries of layers of the structure, retains its form, however, the expressions for $\mathbf{D}, \mathbf{P}^{(\mathrm{U})}$ and $\mathbf{P}^{(\mathrm{D})}$, included in the equation, change. In this case,

$$
\begin{aligned}
& \mathbf{D}=\mathbf{W}_{i n c} A_{i n c}, \\
& \mathbf{P}^{(U)}=\mathbf{W}_{U}^{(-)}, \\
& \mathbf{P}^{(D)}=\mathbf{W}_{D}^{(+)} .
\end{aligned}
$$

As before, for obtaining a numerically-stable solution of equations (1.71) and finding the amplitudes of the transmitted and reflected waves we can use the scattering matrix algorithm, described in section 1.2.6. For the calculation of the distribution of the field we can use a stable procedure described in subsection 1.2.7. 


\subsubsection{The energy characteristics of the reflected and transmitted light}

First we find the energy characteristics of the transmitted radiation. To do this, we calculate the flux of the $z$-component of the Umov-Poynting vector $S_{z}=\operatorname{Re}\left(E_{x} H_{y}^{*}-E_{y} H_{x}^{*}\right) / 2$ of the transmitted waves within a period through the segment $z=z_{L}$. We rewrite the representation of the field (1.167) for $z=z_{L}$, separating parts of the eigenvectors $\mathbf{W}_{D}^{(+)}$corresponding to the various components of the electromagnetic field:

$$
\left[\begin{array}{c}
\mathbf{S}_{y}^{D} \\
\mathbf{S}_{x}^{D} \\
\mathbf{U}_{y}^{D} \\
\mathbf{U}_{x}^{D}
\end{array}\right]=\left[\begin{array}{c}
\mathbf{W}_{D, \mathbf{S}_{y}}^{(+)} \\
\mathbf{W}_{D, \mathbf{S}_{x}}^{(+)} \\
\mathbf{W}_{D, \mathbf{U}_{y}}^{(+)} \\
\mathbf{W}_{D, \mathbf{U}_{x}}^{(+)}
\end{array}\right] \mathbf{T} .
$$

Taking into account (1.169) in (1.27) and assuming for the sake of simplicity that $y=0$, we obtain:

$$
\left\{\begin{array}{l}
E_{x}=\sum_{k} t_{k} \sum_{j} w_{j, k, S_{x}} \exp \left(i k_{x, j} x\right), \\
E_{y}=\sum_{k} t_{k} \sum_{j} w_{j, k, S_{y}} \exp \left(i k_{x, j} x\right), \\
H_{x}=-i \sum_{k} t_{k} \sum_{j} w_{j, k, U_{x}} \exp \left(i k_{x, j} x\right), \\
H_{y}=-i \sum_{k} t_{k} \sum_{j} w_{j, k, U_{y}} \exp \left(i k_{x, j} x\right)
\end{array}\right.
$$

where $t_{i}$ are the elements of the vector $\mathbf{T}, w_{j, i, S_{x}}, w_{j, i, S_{y}}, w_{j, i, U_{x}}$, and $w_{j, i, U_{y}}$ are the elements of vectors $\mathbf{W}_{D, \mathbf{s}_{x}}^{(+)}, \mathbf{W}_{D, \mathbf{s}_{y}}^{(+)}, \mathbf{W}_{D, \mathbf{U}_{x}}^{(+)}$, and $\mathbf{W}_{D, \mathbf{U}_{y}}^{(+)}$ respectively. Taking into account (1.170) in the expression for the z-component of the Umov-Poynting vector, we get:

$$
S_{d}^{D}=2 \int_{0}^{d} S_{z} d x=\sum_{k, m, j, n} \int_{0}^{d} \operatorname{Re}\left(\begin{array}{l}
i t_{k} t_{m}^{*}\left[w_{j, k, S_{x}} w_{n, m, U_{y}}^{*}-w_{j, k, S_{y}} w_{n, m, U_{x}}^{*}\right] \\
\exp \left[i\left(k_{x, j}-k_{x, n}\right) x\right]
\end{array}\right) d x
$$

Given the equality $\int_{0}^{d} \operatorname{Re}\left(a \exp \left[i\left(k_{x, j}-k_{x, n}\right) x\right]\right) d x=\operatorname{Re}(a) d \delta_{j n}$ from (1.171) we obtain: 


$$
S_{d}^{D}=d \sum_{k, m} \operatorname{Im}\left(t_{k} t_{m}^{*} \sum_{j}\left[w_{j, k, S_{y}} w_{j, m, U_{x}}^{*}-w_{j, k, S_{x}} w_{j, m, U_{y}}^{*}\right]\right) .
$$

From (1.172) it follows that, in contrast to the above-mentioned periodic structures, the flux of the Umov-Poynting vector in the period can not be represented as a sum of terms, each of which corresponds to a single wave. Note, however, that the waveguide and plasmonic modes, propagating in the original structure, the following condition of orthogonality is numerically satisfied (up to the order of $10^{-10}$ ):

$$
\sum_{j}\left[w_{j, k, S_{y}} w_{j, m, U_{x}}^{*}-w_{j, k, S_{x}} w_{j, m, U_{y}}^{*}\right]=0, k \neq m,
$$

where $k$ is a fixed number, and $m$ runs through all values from 1 to the number of waves corresponding to the dimensionality of the system of differential equations (1.35). Verification of fulfillment of this condition allows us to judge the correctness of the choice of the values of the period $d$ for artificial periodization and the parameters of absorbing layers. But the waves, for which the orthogonality condition (1.173) is fulfilled, can be given the energy characteristics similar to the intensity of the diffraction orders and determined by expression

$$
I_{n}^{T}=\frac{\left|t_{n}\right|^{2}\left|\operatorname{Im}\left(\sum_{j}\left[w_{j, n, S_{y}} w_{j, n, U_{x}}^{*}-w_{j, n, S_{x}} w_{j, n, U_{y}}^{*}\right]\right)\right|}{\left|A_{i n c}\right|^{2}\left|\operatorname{Im}\left(\sum_{j}\left[w_{j, i n c, S_{y}} w_{j, i n c, U_{x}}^{*}-w_{j, \text { inc }, S_{x}} w_{j, i n c, U_{y}}^{*}\right]\right)\right|},
$$

where $w_{j, i n c, S_{x}}, w_{j, i n c, S_{y}}, w_{j, i n c, U_{x}}$, and $w_{j, i n c, U_{y}}$ are the elements of the vector $\mathbf{W}_{\text {inc }}$. The denominator of (1.174) gives the flux of the Umov-Poynting vector of the incident wave. Similarly, the energy characteristics of the reflected waves are calculated: the elements of the vector $\mathbf{T}$ (1.174) are replaced by the elements of the vector $\mathbf{R}$, and the elements of the eigenvectors from the matrix $\mathbf{W}_{D}^{(+)}$are replaced by the corresponding values from the matrix $\mathbf{W}_{D}^{(-)}$.

\subsubsection{Numerical example}

Consider as an example the propagation of a TM-polarized 
fundamental mode of the plane-parallel waveguide through a couple of grooves in it (Fig. 1.9) [21]. The refractive indices of the materials are shown in the Fig., the waveguide thickness is $300 \mathrm{~nm}$, the width of the grooves and the step separating them are $150 \mathrm{~nm}$. The wavelength of the radiation in free space is $975 \mathrm{~nm}$.

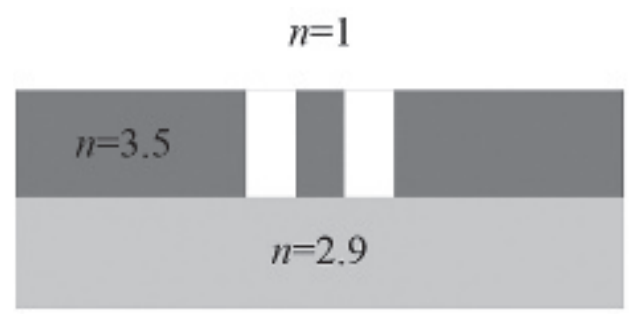

Fig. 1.9. The geometry of the diffraction problem.

Table 1.1 shows the values of the coefficients of reflection and transmission (energy characteristics of the reflected and transmitted modes) depending on the number of harmonics $N$ (the total number of harmonics is $2 N+1$ ). Simulation was carried out using perfectly matched absorbing layers in the form of coordinate transformations. The following simulation parameters were: $e=900 \mathrm{~nm}, d=3 e, \gamma=$ $1 /(1-i)$, the middle of the waveguide coincided with the middle of the period.

Table 1.1. Coefficients of reflection and transmission for the TM-polarized fundamental mode

\begin{tabular}{lll}
\hline$N$ & $I^{R}$ & $I^{T}$ \\
\hline 50 & 0.356216 & 0.129970 \\
100 & 0.355637 & 0.129675 \\
150 & 0.355569 & 0.129619 \\
200 & 0.355531 & 0.129604 \\
\hline
\end{tabular}

\subsection{Methods for calculating eigenmodes of periodic diffractive structures}

\subsubsection{Calculation of modes based on the calculation of the poles of the scattering matrix}

The diffractive micro- and nanostructures with the resonant properties are of great interest for designing of modern elements of integrated optics and photonics [19,24-31]. The resonant properties are manifested in the abrupt change in the transmission and reflection spectra, and are usually associated with the excitation of the 
eigenmodes of the structure. The eigenmodes can be calculated as the poles of the scattering matrix $[28,32]$. Such an approach to the explanation of the optical properties of diffractive structures is widely used to describe the optical properties of diffraction gratings [28, 32], including those containing anisotropic [33] and gyrotropic [29] materials, photonic crystal structures $[30,31]$ and laser resonators [19].

Calculation of the poles of the scattering matrix is a computationally difficult task. The known papers propose several iterative methods for solving it. The simplest method is to calculate the poles of the determinant of the scattering matrix $[30,31]$ or the poles of its maximum eigenvalue [34]. In [26,27,35] the authors proposed a more sophisticated method based on the linearization of the scattering matrix inverse. To adequately describe the optical properties of the metal-dielectric diffraction gratings and plasmonic structures it is required to use the scattering matrix with high dimensions. Typically, the scattering matrix is calculated on the basis of thr Fourier modal method [2]. Despite the variety of approaches to improving the convergence of the method, the scattering matrix dimensions may amount to several hundreds [36]. Calculations of the determinant and the inverse scattering matrix for such large matrices carried out in $[26,27,31,34,35]$ become numerically unstable [37], significantly limiting the area of applicability of the methods.

We should also highlight the method for calculating the frequency of modes, based on Cauchy's integral formula. This method is used in calculating the modes propagating in plane-parallel waveguides $[38,39]$ and photonic crystal structures [34,40,41]. The distinctive feature of this method is that it allows one to find all the poles of the scattering matrix, located in a given area [34,40,41]. However, in these studies the authors made a number of assumptions about the form of the scattering matrix which usually do not take place for the scattering matrix of a diffraction grating.

In this section, we compare the known iterative methods of calculating the scattering matrix poles and propose, in our opinion, a new method, which takes into account the form of the scattering matrix in the vicinity of resonances and has better convergence. In this section we also proposes a modification of the method based on Cauchy's integral formula which generalizes the methods of [24-26].

\subsubsection{Resonant representation of the scattering matrix}

Consider the scattering matrix of a periodic structure. Let $N^{\prime}=$ 
$2 N+1$ denote the number of Fourier harmonics considered in the representation of the field (1.27). In this case, the scattering matrix relates $2 N^{\prime}$ incident and $2 N^{\prime}$ scattered waves. Taking into account the two polarization states of the incident and scattered waves, the size of the scattering matrix is $4 N^{\prime} \times 4 N^{\prime}[28,2]$. For the given geometry and grating materials the scattering matrix $\mathbf{S}$ is a function of frequency $\omega$ and the $x$-component of the wave vector of the incident wave with the number $m=0: \mathbf{S}=\mathbf{S}\left(\omega, k_{x, 0}\right)$. In order to describe the resonances in the transmission or reflection spectrum of the grating we fix the direction of the incident wave, and consider the scattering matrix as a function of frequency $\omega: \mathbf{S}=\mathbf{S}(\omega)$. The resonances of the structure correspond to the poles of the analytic continuation of $\mathbf{S}(\omega)$ [19]. The real part of the poles corresponds to the frequency of the incident wave that can excite a mode, while the imaginary part determines mode decay.

Consider the analytic continuation $\mathbf{S}(\omega), \omega \in \mathbb{C}$ of the scattering matrix for the domain $\mathrm{D}$ of the complex $\omega$-plane bounded by a closed curve $\Gamma$. We assume that the Rayleigh anomalies of the structure are located far from the frequency region of interest. In this case, the analytic continuation $\mathbf{S}(\omega)$ in the considered domain D is singlevalued $[42,43]$.

Suppose the analytic continuation of the scattering matrix in domain D has a simple pole at $\omega=\omega_{\mathrm{p}}$. Next, we consider the case when the poles of the scattering matrix are simple. This assumption is valid for problems of electrodynamics and quantum mechanics [40]. In this case, it makes sense to determine the residue of the scattering matrix $\mathbf{S}(\omega)$ :

$$
\operatorname{Res}_{\omega=\omega_{p}} \mathbf{S}(\omega)=\frac{1}{2 \pi i} \oint_{\gamma} \mathbf{S}(\omega) d \omega
$$

where the integration contour $\gamma$ is chosen in such a way that it contains only one pole $\omega_{\mathrm{p}}$. Equation (1.175) should be understood as an elementwise operation. If the scattering matrix has only one pole in domain $\mathrm{D}$, then the following representation holds:

$$
\mathbf{S}(\omega)=\mathbf{A}(\omega)+\frac{\mathbf{B}}{\omega-\omega_{\mathrm{p}}}
$$


where $\mathbf{B}=\operatorname{Res}_{\omega=\omega_{p}} \mathbf{S}(\omega)$, and the matrix-valued function $\mathbf{A}(\omega)$ has no poles in the domain D and is holomorphic in this area. In general, when domain D contains $M$ poles, the expansion (1.176) takes the following form:

$$
\mathbf{S}(\omega)=\mathbf{A}(\omega)+\sum_{m=1}^{M} \frac{\mathbf{B}_{m}}{\omega-\omega_{\mathrm{p}}^{(m)}}
$$

where $B_{m}=\underset{\omega=\omega_{\mathrm{p}}^{(m)}}{\operatorname{Res}} \mathbf{S}(\omega)$. The first term in (1.176) and (1.177) describes the non-resonant scattering, the second one - the resonant scattering.

We now consider the properties of the matrix $\mathbf{S}(\omega)$. Assume that $\mathbf{S}(\omega)$ has a pole at $\omega=\omega_{\mathrm{p}}$, and the elements of the inverse matrix $\mathbf{S}^{-1}(\omega)$ do not have a pole at a given frequency. In this case, the kernel of the matrix $\mathbf{S}^{-1}\left(\omega_{p}\right)$ determines the non-trivial solutions of the homogeneous equation:

$$
\mathbf{S}^{-1} \cdot \Psi^{\text {scatt }}=\mathbf{0},
$$

where, according to (1.86), $\Psi^{\text {scatt }}=\left[\begin{array}{l}\mathbf{T} \\ \mathbf{R}\end{array}\right]$.

Thus, $\operatorname{ker} \mathbf{S}^{-1}\left(\omega_{\mathrm{p}}\right)$ describes the distribution of the field that exists in the absence of incident waves, that is, the frequency $\omega_{\mathrm{p}}$ corresponds to the frequency of the quasi-guided grating mode.

It is easy to show that $\operatorname{ImB}=\operatorname{ker} \mathbf{S}^{-1}\left(\omega_{\mathrm{p}}\right)$, so $\operatorname{rank} \mathbf{B}=$ $\operatorname{dim} \operatorname{ker}^{-1}$ [40]. Usually $\operatorname{rank} \mathbf{B}=1$, i.e. the same frequency matches only one mode. However, under certain parameters of the structure the frequencies of several different modes may coincide. In this case rankB $>1$. Such resonances are called degenerate. Structures with degenerate resonances have interesting optical properties [24,25].

Suppose that in a general case $\operatorname{rank} \mathbf{B}>r$. In this case, for the matrix $\mathbf{B}$ we can write the rank factorization [44]

$$
\mathbf{B}=\mathbf{L R},
$$

where $\mathbf{L} \in \mathbb{C}^{n \times r}, \mathbf{R} \in \mathbb{C}^{r \times n}, \operatorname{rank} \mathbf{L}=\operatorname{rank} \mathbf{R}=r$. Taking into account (1.179), the expression (1.177) can be written as

$$
\mathbf{S}=\mathbf{A}(\omega)+\mathbf{L} \frac{1}{\omega-\omega_{\mathrm{p}}} \mathbf{R} .
$$

The general expansion (1.177) can be written as follows: 


$$
\mathbf{S}=\mathbf{A}(\omega)+\sum_{m=1}^{M} \mathbf{L}_{m} \frac{1}{\omega-\omega_{\mathrm{p}}^{(m)}} \mathbf{R}_{m}=\mathbf{A}(\omega)+\mathbf{L}\left(\mathbf{I} \omega-\mathbf{\Omega}_{\mathrm{p}}\right)^{-1} \mathbf{R},
$$

where $\mathbf{L}, \mathbf{R}$ are the block matrices $\mathbf{L}=\left[\mathbf{L}_{1}, \mathbf{L}_{2} \ldots \mathbf{L}_{M}\right], \mathbf{R}^{\mathrm{T}}=$ $\left[\begin{array}{llll}\mathbf{R}_{1}^{\mathrm{T}} & \mathbf{R}_{2}^{\mathrm{T}} & \cdots & \mathbf{R}_{M}^{\mathrm{T}}\end{array}\right]$ and $\boldsymbol{\Omega}_{\mathrm{p}}$ is a diagonal matrix composed of $\omega_{\mathrm{p}}^{(m)}$, $m=1 \ldots M$, with the number of repetitions of frequency $\omega_{\mathrm{p}}^{(m)}$ equal to the rank of the corresponding matrix $\mathbf{B}_{m}$. Equations (1.180) and (1.181) determine the resonant representation of the scattering matrix, which will be used in the further derivation of numerical methods for the calculation of the scattering matrix poles.

\subsubsection{Calculation of the poles of the scattering matrix}

As shown above, the poles of the scattering matrix determine the eigenmodes of the structure. Consider the problem of calculating the poles of the scattering matrix $\mathbf{S}(\omega)$ in domain $\mathrm{D}$. The easiest method is to move from the problem of calculating the poles of the matrixvalued function $\mathbf{S}(\omega)$ to the problem of finding the poles of a scalar function $\operatorname{det} \mathbf{S}(\omega)$, that is, to the numerical solution of the equation

$$
1 / \operatorname{det} \mathbf{S}(\omega)=0 .
$$

This method allows us to calculate the poles for a small dimensional matrix $\mathbf{S}$. When a large dimensional matrix $\mathbf{S}(\omega)$ is considered the calculation of the determinant becomes numerically unstable. A more approach consists in solving the following equation [34]:

$$
1 / \max \operatorname{eig} \mathbf{S}(\omega)=0 .
$$

where max eig $\mathbf{S}(\omega)$ is the eigenvalue of the matrix $\mathbf{S}(\omega)$ having the maximal modulus. To solve the equations (1.182) and (1.183) we can use iterative methods for finding the root of the non-linear equation, such as Newton's method, or a more general Householder's method [45]. For equation (1.183), this method can be written as the following iterative procedure:

$$
\omega_{n+1}=\omega_{n}+\left.p \frac{\frac{\mathrm{d}^{p-1}}{\mathrm{~d} \omega^{p-1}} \max \operatorname{eig} \mathbf{S}(\omega)}{\frac{\mathrm{d}^{p}}{\mathrm{~d} \omega^{p}} \max \operatorname{eig} \mathbf{S}(\omega)}\right|_{=\omega_{n}}
$$


where $\omega_{n}$ is an initial approximation of the pole. When $p=1$ the equation (1.184) corresponds to Newton's method, when $p=2$ to Halley's method. In solving the equations (1.182) and (1.183) the method (1.184) does not take into account the form of the scattering matrix (1.181), replacing it with a scalar integer (either with the determinant or with the maximum modulus eigenvalue $\mathbf{S}(\omega)$ ). This is the reason for the relatively slow convergence of the above methods. In the following sections we consider the iterative methods of calculation of the poles of the scattering matrix using the matrix expansion.

\section{Calculation of the poles based on linearization of the scattering matrix inverse}

Consider an iterative method of calculation of the poles of the scattering matrix proposed in $[19,32,33,34]$. Let $\omega_{n}$ be some initial approximation of the pole. We expand the matrix $\mathbf{S}^{-1}(\omega)$ into a Taylor series up to the first order term

$$
\mathbf{S}^{-1}(\omega)=\mathbf{S}^{-1}\left(\omega_{n}\right)+\left.\frac{\mathrm{d} \mathbf{S}^{-1}}{\mathrm{~d} \omega}\right|_{\omega_{n}}\left(\omega-\omega_{n}\right) .
$$

Let $\omega_{\mathrm{p}}$ be the pole of the scattering matrix, in this case there exists the vector $\Psi^{\text {scatt }}$ which is a non-trivial solution of (1.178). Multiplying (1.185) by $\boldsymbol{\Psi}^{\text {scatt }}$ on the right side when $\omega=\omega_{\mathrm{p}}$ we have:

$$
\mathbf{S}^{-1}\left(\omega_{n}\right) \Psi^{\text {scatt }}=\left.\left(\omega_{n}-\omega_{\mathrm{p}}\right) \frac{\mathrm{d} \mathbf{S}^{-1}}{\mathrm{~d} \omega}\right|_{\omega_{n}} \boldsymbol{\Psi}^{\text {scatt }}
$$

Equation (1.186) is a generalized eigenvalue problem. Solving this problem, we obtain a set of eigenvalues $\lambda_{k}=\omega_{n}-\omega_{\mathrm{p}}$. Choosing from the eigenvalues the number with a minimal modulus, we obtain the following iterative procedure:

$$
\omega_{n+1}=\omega_{n}-\min \operatorname{eig}\left(\mathbf{S}^{-1}\left(\omega_{n}\right),\left.\frac{\mathrm{d} \mathbf{S}^{-1}}{\mathrm{~d} \omega}\right|_{\omega_{n}}\right)
$$

where $\operatorname{eig}(\mathbf{F}, \mathbf{G})$ denotes the vector composed of the eigenvalues for a generalized eigenvalue problem $\mathbf{F} \cdot \mathbf{X}=\lambda \mathbf{G} \cdot \mathbf{X}$. Selecting in (1.187) the eigenvalue with the minimum modulus means that in the next 
approximation of the pole $\omega_{n+1}$ we select the closest approximation to the original value $\omega_{n}$.

We note a number of shortcomings of the method (1.187). The main drawback is the inability to carry out numerically stable computation of the matrix inverse to the scattering matrix for a large number of orders. [37]. Second, note that if in the vicinity of the pole of the scattering matrix $\mathbf{S}(\omega)$ there is a pole of the matrix $\mathbf{S}^{-1}(\omega)$, the Taylor series in (1.185) will not converge [46].

Solution of the system of matrix equations $A=L X R, B=L Y R$

Before proceeding to the description of more advanced methods for calculating the poles of the scattering matrix, based on the resonance approximation, we consider the necessary mathematical apparatus. Let us assume we have matrices $\mathbf{A}, \mathbf{B} \in \mathbb{C}^{n \times n}$ that have the following form:

$$
\left\{\begin{array}{l}
\mathbf{A}=\mathbf{L X R} \\
\mathbf{B}=\mathbf{L Y R},
\end{array}\right.
$$

where $\mathbf{L} \in \mathbb{C}^{n \times r}, \mathbf{R} \in \mathbb{C}^{r \times n}$ are some unknown matrices; $\mathbf{X} \in \mathbb{C}^{r \times r}$, $\mathbf{Y} \in \mathbb{C}^{r \times r}$ are unknown diagonal matrices, with the value of $r$ is also unknown. We assume that $\operatorname{rank} \mathbf{A}=r$. It is required to find the connection between the matrices $\mathbf{X}$ and $\mathbf{Y}$. Note that systems of this kind occur in the problem of determining the poles of LTI-systems $[47,48]$.

Consider the method of solving the system (1.188), based on the singular value decomposition [49]. First, we find the rank $r$ of the matrix A. To do this, we calculate its singular value decomposition $\mathbf{A}=\mathbf{U} \boldsymbol{\Sigma} \mathbf{V}^{*}$ (here $\mathbf{V}$ denotes the Hermitian conjugation). Rank $r$ is determined by the number of non-zero singular numbers. In practice, the matrix $\mathbf{A}$ can be noisy, in this case $r$ should be taken as the number of singular values greater than a certain threshold. Knowing the rank $\mathbf{A}$, we can write compact singular value expansion:

$$
\mathbf{A}=\mathbf{U}_{r} \boldsymbol{\Sigma}_{r} \mathbf{V}_{r} *
$$

where $\Sigma_{r} \in \mathbb{R}^{r \times r}$ is a diagonal matrix composed of $r$ largest singular values $\mathbf{A} ; \mathbf{U}_{r}, \mathbf{V}_{r} \in \mathbb{C}^{n \times r}$ are the matrices of the corresponding left and right singular vectors. In the case of the noised matrix the equation (1.189) holds approximately and it makes sense to approximate the matrix A with the matrix of rank $r$.

Note that the matrices $\mathbf{U}$ and $\mathbf{V}$ are unitary and hence $\mathbf{U}_{r}^{*} \mathbf{U}_{r}=\mathbf{V}_{r}^{*} \mathbf{V}_{r}=\mathbf{1}$. We multiply the equations of the system (1.188) on the left by $\mathbf{U}_{r}^{*}$ and on the right by $\mathbf{V}_{r}$. We obtain 


$$
\begin{aligned}
& \left(\mathbf{U}_{r}^{*} \mathbf{L}\right) \mathbf{X}\left(\mathbf{R} \mathbf{V}_{r}\right)=\left(\mathbf{U}_{r}^{*} \mathbf{U}_{r}\right) \boldsymbol{\Sigma}_{r}\left(\mathbf{V}_{r}^{*} \mathbf{V}_{r}\right)=\Sigma_{r} ; \\
& \left(\mathbf{U}_{r}^{*} \mathbf{L}\right) \mathbf{Y}\left(\mathbf{R} \mathbf{V}_{r}\right)=\mathbf{U}_{r}^{*} \mathbf{B} \mathbf{V}_{r} .
\end{aligned}
$$

Note $\operatorname{rank} \boldsymbol{\Sigma}_{r}=\operatorname{rank} \mathbf{A}=\operatorname{rank} \mathbf{U}_{r}=\operatorname{rank} \mathbf{V}_{r}=r$ hence $\mathbf{U}_{r}^{*} \mathbf{L}$ and $\mathbf{R} \mathbf{V}_{r}$ are the invertible matrices from matrices $\mathbb{C}^{r \times r}$. Multiplying the second equation (1.190) by $\Sigma_{r}^{-1}$ on the right, we get:

$$
\left(\mathbf{U}_{r}^{*} \mathbf{L}\right) \mathbf{Y} \mathbf{X}^{-1}\left(\mathbf{U}_{r}^{*} \mathbf{L}\right)^{-1}=\mathbf{U}_{r}^{*} \mathbf{B} \mathbf{V}_{r} \Sigma_{r}^{-1}
$$

Equation (1.191) can be considered as eigendecomposition of the matrix on the right-hand side of the equation. Thus, we found an explicit expression for the matrix $\mathbf{Y} \mathbf{X}^{-1}$ :

$$
\mathbf{Y} \mathbf{X}^{-1}=\operatorname{diag} \operatorname{eig}\left(\mathbf{U}_{r}^{*} \mathbf{B} \mathbf{V}_{r} \boldsymbol{\Sigma}_{r}^{-1}\right)
$$

Consider the important special case when the matrices $\mathbf{A}, \mathbf{B}$ are the matrix of unit rank, that is $r=1$. In this case, the vector $\mathbf{U}_{1}$ is an eigenvector of the matrix $\mathbf{A}$, and $\mathbf{V}_{1}$ the eigenvector of matrix $\mathbf{A}^{*}$. Consequently, the matrix $\mathbf{A}$ can be represented as

$$
\mathbf{A}=\mathbf{U}_{1} \frac{\max \operatorname{eig} \mathbf{A}}{\mathbf{V}_{1}^{*} \mathbf{U}_{1}} \mathbf{V}_{1}^{*}
$$

where maxeig $\mathbf{A}$ is the unique non-zero eigenvalue of matrix $\mathbf{A}$. Vectors $\mathbf{U}_{1}, \mathbf{V}_{1}$ are also eigenvectors of the matrices $\mathbf{B}$ and $\mathbf{B}^{*}$, respectively. Therefore we have

$$
\mathbf{B}=\mathbf{U}_{1} \frac{\max \operatorname{eig} \mathbf{B}}{\mathbf{V}_{1}^{*} \mathbf{U}_{1}} \mathbf{V}_{1}^{*}
$$

By substituting (1.193) and (1.194) into (1.192), we obtain:

$$
y / x=\frac{\max \operatorname{eig} \mathbf{B}}{\max \operatorname{eig} \mathbf{A}} .
$$

Note that in practice the matrices $\mathbf{A}$ and $\mathbf{B}$ can be noised and therefore in formula (1.195) one should take eigenvalue of maximum modulus.

Calculation of the poles on the basis of the resonance approximation Consider an iterative method of calculation of the poles of the scattering matrix, taking into account the form of the scattering matrix in the vicinity of resonances. Let there be some initial 
approximation of the pole $\omega=\omega_{n}$. We write resonance approximation (1.181), neglecting the nonresonant term dependence on frequency:

$$
\mathbf{S}(\omega)=\mathbf{A}+\mathbf{L}\left(\omega \mathbf{I}-\boldsymbol{\Omega}_{\mathrm{p}}\right)^{-1} \mathbf{R} .
$$

We calculate the first two derivatives $\mathbf{S}(\omega)$ :

$$
\begin{aligned}
& \mathbf{S}^{\prime}(\omega)=-\mathbf{L}\left(\omega \mathbf{I}-\boldsymbol{\Omega}_{\mathrm{p}}\right)^{-2} \mathbf{R}, \\
& \mathbf{S}^{\prime \prime}(\omega)=2 \mathbf{L}\left(\omega \mathbf{I}-\boldsymbol{\Omega}_{\mathrm{p}}\right)^{-3} \mathbf{R} .
\end{aligned}
$$

We assume that $\operatorname{rank} \boldsymbol{L}=\operatorname{rank} \boldsymbol{R}=\Sigma_{m} \operatorname{rank} \boldsymbol{L}_{m}=\Sigma_{m} \operatorname{rank} \boldsymbol{R}_{m}$. This assumption suggests that the columns of the matrix $\mathbf{L}$ are linearly independent or, which is the same, the kernels of the matrices $\mathbf{S}^{-1}\left(\omega_{\mathrm{p}}^{(m)}\right), m=1 \ldots M$ are linearly independent. This means that the distribution of the scattered fields for different modes are linearly independent. This assumption is usually performed when the size of the scattering matrix is much larger than the number of modes $(\operatorname{dim} \mathbf{S}>\operatorname{rank} \mathbf{L})$.

Assuming in (1.197) $\omega=\omega_{n}$, we obtain a system of two matrix equations for the unknown diagonal matrix $\boldsymbol{\Omega}_{\mathrm{p}}$. A method for solving systems of the form (1.197) is given in the preceding paragraph. Following this method (see (1.192)), the diagonal matrix $\boldsymbol{\Omega}_{\mathrm{p}}$ is calculated according to the formula:

$$
\boldsymbol{\Omega}_{\mathrm{p}}=\omega_{n} \mathbf{I}+2 \operatorname{diag} \operatorname{eig}\left(\mathbf{U}_{r}^{*} \mathbf{S}^{\prime}\left(\omega_{n}\right) \mathbf{V}_{r} \boldsymbol{\Sigma}_{r}^{-1}\right),
$$

where diag eig $\mathbf{F}$ is a diagonal matrix composed of the eigenvalues $\mathbf{F}$ and the matrix $\mathbf{U}_{r}, \Sigma_{r}, \mathbf{V}_{r}$ obtained from the singular value compact decomposition of matrix $\mathbf{S}^{\prime \prime}\left(\omega_{n}\right)=\mathbf{U}_{r} \Sigma_{r} \mathbf{V}_{r}^{*}$. Calculating the eigenvalues of the right-hand side of (1.198), we arrive at the following iterative procedure:

$$
\omega_{n+1}=\omega_{n}+2 \min \operatorname{eig}\left(\mathbf{U}_{r}^{*} \mathbf{S}^{\prime}\left(\omega_{n}\right) \mathbf{V}_{r} \boldsymbol{\Sigma}_{r}^{-1}\right) .
$$

Similar to (1.187), the choice of the minimum-modulus eigenvalue (1.199) means that as the next approximation of the pole $\omega_{n+1}$ we select the closest approximation to the original $\omega_{n}$.

The iterative procedure (1.199) suggests that in the vicinity of the initial approximation there are several poles. In practice, we can assume that in the neighborhood of $\omega_{n}$ there is only one pole corresponding to the non-degenerate resonance. In this case, using the ratio (1.195), iterative procedure takes the following simple form: 


$$
\omega_{n+1}=\omega_{n}+2 \frac{\max \operatorname{eig} \mathbf{S}^{\prime}\left(\omega_{n}\right)}{\max \operatorname{eig} \mathbf{S}^{\prime \prime}\left(\omega_{n}\right)}
$$

Unlike (1.187), the iterative methods (1.199) and (1.200) are based on the resonance approximation (1.196) and not on the linearization of the matrix $\mathbf{S}^{-1}(w)$. The results of numerical investigations in the following paragraphs show that the iterative methods (1.199) and (1.200) have a better convergence than (1.187). In addition, the advantage of the proposed approach is that it remains valid for the case of a large number of diffraction orders (large dimension of the matrix S) in the case when the poles of the scattering matrix and of the scattering matrix inverse.

Note that the method (1.187) has the meaning of Newton's method for matrix-valued functions, while the methods (1.199) and (1.200) can be considered as a generalization of Halley's matrix for solving equations of the form $1 / f(x)=0$. In general, we can write the matrix analogue of the Householder method [45], based on derivatives of the scattering matrix of order $p$ and $p-1\left(\mathbf{S}^{(p)}\left(\omega_{n}\right), \mathbf{S}^{(p-1)}\left(\omega_{n}\right)\right)$. In this case, the iterative procedure (1.199) is a special case $(p=2)$ of the following iterative procedure

$$
\omega_{n+1}=\omega_{n}+p \min \operatorname{eig}\left(\mathbf{U}_{r}^{*} \mathbf{S}^{(p-1)}\left(\omega_{n}\right) \mathbf{V}_{r} \boldsymbol{\Sigma}_{r}^{-1}\right),
$$

where the matrices $\mathbf{U}_{r}, \Sigma_{r}, \mathbf{V}_{r}$ are determined by the compact singular value decomposition of the matrix $\mathbf{S}^{(p)}\left(\omega_{n}\right)=\mathbf{U}_{r} \Sigma_{r} \mathbf{V}_{r}^{*}$. An analogue of the procedure (1.200) is written similarly:

$$
\omega_{n+1}=\omega_{n}+p \frac{\max \operatorname{eig} \mathbf{S}^{(p-1)}\left(\omega_{n}\right)}{\max \operatorname{eig} \mathbf{S}^{(p)}\left(\omega_{n}\right)} .
$$

The last expression is reminiscent of the Householder method (1.184) for the solution of equation (1.183) with the exception that the operations of differentiation and calculation of the maximum eigennumber are swapped. At $p=1$ the methods (1.201) and (1.202) will be the analogue of Newton's method, but, in contrast to (1.187), this method is based on calculating the scattering matrix $\mathbf{S}(\omega)$ and its derivative, not the matrix $\mathbf{S}^{-1}(\omega)$.

\section{Calculation of the poles based on Cauchy's integral formula}

The efficiency of the above methods (1.182), (1.183), (1.187), (1.199) and (1.200) depends significantly on the initial approximation of 
the pole $\omega=\omega_{n}$. In addition, these methods allow one to find only one pole in the vicinity of the initial approximation. Note that the calculation methods of the poles by (1.187), (1.199) and (1.201) can use all the eigenvalues when computing these approximations. However, this approach does not guarantee that all the poles of the scattering matrix can be calculated in the region of interest D.

The following is an approach based on Cauchy's integral to calculate all the poles of the scattering matrix in a given area. This approach is widely used in the calculation of modes of multilayer slab waveguides $[38,39]$ and photonic waveguides $[34,40]$.

Following $[24,25,26]$, we calculate two contour integral of matrixvalued functions:

$$
\begin{aligned}
& \mathbf{C}_{1}=\frac{1}{2 \pi \mathrm{i}} \oint_{\Gamma} \mathbf{S}(\omega) \mathrm{d} \omega, \\
& \mathbf{C}_{2}=\frac{1}{2 \pi \mathrm{i}} \oint_{\Gamma} \omega \mathbf{S}(\omega) \mathrm{d} \omega,
\end{aligned}
$$

where $\Gamma$ is the boundary of region D. Using the representation (1.177) it is easy to get

$$
\begin{aligned}
& \mathrm{C}_{1}=\sum_{\mathrm{n}} \mathrm{B}_{\mathrm{n}} ; \\
& \mathrm{C}_{2}=\sum_{\mathrm{n}}^{\mathrm{n}} \omega_{\mathrm{p}}^{(\mathrm{n})} \mathrm{B}_{\mathrm{n}},
\end{aligned}
$$

where $\mathbf{B}_{n}=\underset{\omega=\omega_{\mathrm{p}}^{(n)}}{\operatorname{Res}} \mathbf{S}(\omega)$. Note that in [40] several examples are used to demonstrate the possibility of finding all the poles, bounded by contour $\Gamma$, on the basis of the integrals (1.203). It should be noted that in [40] the matrices $\mathbf{B}_{n}$ are symmetric and the columns of the matrix $\mathbf{L}$ (1.181) are orthogonal. These assumptions generally do not hold for the scattering matrices of diffraction gratings [27].

Consider the method for solving equations (1.204) under more general assumptions. For this we write (1.204) in the notation of (1.181):

$$
\begin{aligned}
& \mathbf{C}_{1}=\mathbf{L R} ; \\
& \mathbf{C}_{2}=\mathbf{L} \boldsymbol{\Omega}_{\mathbf{p}} \mathbf{R} .
\end{aligned}
$$

As before, we assume that the columns of the matrix $\mathbf{L}$ are linearly independent. This condition is more common than the orthogonality condition, used in [40]. The system of equations (1.205) can be solved for the unknown diagonal matrix $\boldsymbol{\Omega}_{\mathrm{p}}$ using 
the method described above. Following this method, the matrix $\boldsymbol{\Omega}_{\mathrm{p}}$ is calculated by the formula

$$
\boldsymbol{\Omega}_{\mathrm{p}}=\operatorname{diag} \operatorname{eig}\left(\mathbf{U}_{r}^{*} \mathbf{C}_{2} \mathbf{V}_{r} \boldsymbol{\Sigma}_{r}^{-1}\right)
$$

where the matrices $\mathbf{U}_{r}, \Sigma_{r}, \mathbf{V}_{r}$ are obtained from the compact singular value decomposition of matrix $\mathbf{C}_{1}=\mathbf{U}_{r} \Sigma_{r} \mathbf{V}_{r}^{*}$.

Thus, calculating the integrals (1.203), one can find all the poles of the scattering matrix, located inside the integration contour. The accuracy of calculation of the poles is determined by the accuracy of numerical calculation of integrals (1.203). For calculation of poles with high precision one requires a large number of calculations of the scattering matrix $[34,40]$. Therefore, the method based on Cauchy's integral formula should be applied only to calculate the initial approximations of the poles, while the exact values can be obtained using the iterative procedures discussed above.

\subsubsection{Numerical examples}

This section describes the calculation of the poles of the scattering matrix of the diffraction grating using the above methods. The scattering matrix of the grating was calculated by the Fourier modal method $[2,5,18]$. We note that the condition of analyticity of the scattering matrix requires proper implementation of the Fourier modal method for complex frequencies [28, 35].

Consider the calculation of eigenmodes of the diffraction grating corresponding to the periodic array of slits in a silver film, located on an dielectric waveguide layer (Fig. 1.10). We are interested in the modes which can be excited by a normally incident plane wave $\left(k_{x, 0}=\right.$ $0)$. The grating parameters are shown in the caption to Fig. 1.10. The dielectric constant of silver was described by the Drude-Lorentz model [50], and the analytical expression for $\varepsilon_{\mathrm{Ag}}(\omega)$ is considered as a function of complex frequency. Note that the analytical function $\varepsilon(\omega)$ is a prerequisite for the analyticity of $\mathbf{S}(\omega)$. If this condition is not met, then the convergence rate of the iterative methods significantly deteriorates, and the method based on Cauchy's integral theorem even stops working. Furthermore, we assume that the frequency of the mode of the grating is calculated correctly if $\max \operatorname{svd} \mathbf{S}(\omega) \geq$ $10^{10}$, where max svd is the maximum singular value of the scattering matrix. This inequality will be used as a criterion for stopping the iterative algorithm. Numerical calculations show that this condition 
for the given grating corresponds to the accuracy of determining the poles $\left|\Delta \lambda_{\mathrm{p}}\right|<10^{-8} \mathrm{~nm}$. This accuracy is sufficient for practical applications, including the calculation of the field distribution of the modes.

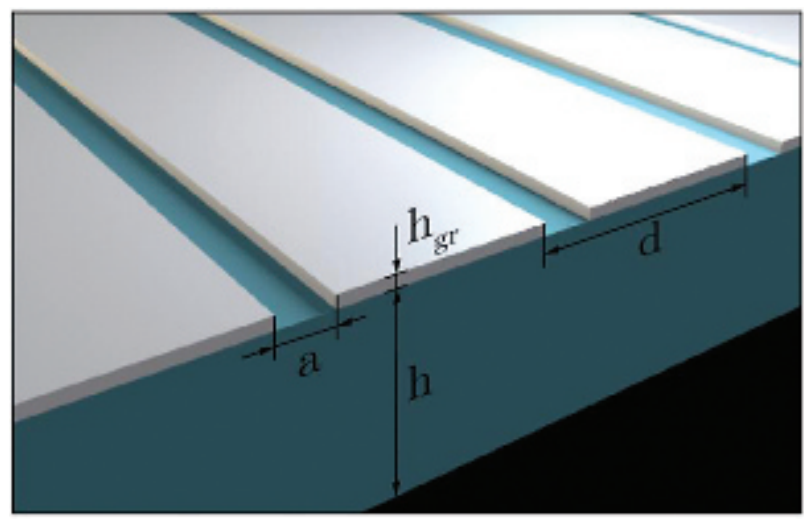

Fig. 1.10. A silver grating on a waveguide layer (grating parameters: period $d=$ $1000 \mathrm{~nm}$, the grating height $h_{\mathrm{gr}}=50 \mathrm{~nm}$, the slit width $a=200 \mathrm{~nm}$, the thickness of the waveguide layer $h=800 \mathrm{~nm}$, the permittivity of the layer $\varepsilon=5.5$ ).

\section{Method based on Cauchy's integral formula}

We find all the modes of the grating in the frequency range $\omega \in\left[1.0 \cdot 10^{15} \mathrm{~s}^{-1} ; 1.8 \cdot 10^{15} \mathrm{~s}^{-1}\right]$. We shall consider only the high-Q modes $\left(\left|\mathrm{Im} \omega_{\mathrm{p}}\right|<5 \cdot 10^{12} \mathrm{~s}^{-1}\right)$. In further calculations, we used the value $N^{\prime}=21$ for the number of Fourier harmonics.

To assess the mode frequencies, we calculated the contour integrals (1.203) using the trapezoidal method with for 500 points. Formula (1.206) was used to obtain 10 approximate values of the poles. These values are listed in the first column of Table 1.2. The second column shows the exact frequency of the poles, calculated using an iterative procedure (1.200). The third column contains the error of initial approximation. The table shows that the calculation of the poles based on the method of contour integration is sufficiently accurate.

For comparison, we note that if we calculate the initial approximations of the poles by the calculation of the scattering matrix on the grid consisting of $500 \times 20$ points $(10000$ calculations of the scattering matrix), we are able to find only 9 poles. This is due to the fact that the poles $\omega_{6}, \omega_{7}$ are very close to each other. Thus, using the method based on Cauchy's integral formula is justified in this case. 
Table 1.2. The poles, calculated by the formula (1.206), and their refinement by different iterative methods

\begin{tabular}{|c|c|c|c|c|c|c|}
\hline & $\omega_{\text {cauchy }}$ & $\omega_{\text {exact }}$ & $\left|\omega_{\text {exact }}-\omega_{\text {cauchy }}\right|$ & $(1.200)$ & $\begin{array}{c}n_{\text {iter }} \\
(1.187)\end{array}$ & (1.182) \\
\hline$\omega_{1}$ & $\begin{array}{l}1.14246 \times 10^{15}- \\
3.35055 \times 10^{12} \mathrm{i}\end{array}$ & $\begin{array}{l}1.14247 \times 10^{15}- \\
3.34402 \times 10^{12} \mathrm{i}\end{array}$ & $7.8665 \times 10^{9}$ & 1 & 2 & 3 \\
\hline$\omega_{2}$ & $\begin{array}{l}1.15554 \times 10^{15}- \\
6.85407 \times 10^{11} \mathrm{i}\end{array}$ & $\begin{array}{l}1.15551 \times 10^{15}- \\
6.86647 \times 10^{12} \mathrm{i}\end{array}$ & $3.40401 \times 10^{10}$ & 2 & 2 & 3 \\
\hline$\omega_{3}$ & $\begin{array}{l}1.40431 \times 10^{15}- \\
1.87394 \times 10^{12} \mathrm{i}\end{array}$ & $\begin{array}{l}1.40428 \times 10^{15}- \\
1.87643 \times 10^{12} \mathrm{i}\end{array}$ & $3.45275 \times 10^{10}$ & 2 & 2 & 3 \\
\hline$\omega_{4}$ & $\begin{array}{l}1.48168 \times 10^{15}- \\
3.86736 \times 10^{12} \mathrm{i}\end{array}$ & $\begin{array}{l}1.48168 \times 10^{15}- \\
3.86743 \times 10^{12} \mathrm{i}\end{array}$ & $2.35895 \times 10^{10}$ & 1 & 2 & 3 \\
\hline$\omega_{5}$ & $\begin{array}{l}1.5047 \times 10^{15}- \\
1.11064 \times 10^{12} \mathrm{i}\end{array}$ & $\begin{array}{l}1.50469 \times 10^{15}- \\
1.11116 \times 10^{12} \mathrm{i}\end{array}$ & $1.52001 \times 10^{10}$ & 2 & 2 & 3 \\
\hline$\omega_{6}$ & $\begin{array}{l}1.66425 \times 10^{15}- \\
7.11868 \times 10^{11} \mathrm{i}\end{array}$ & $\begin{array}{l}1.66425 \times 10^{15}- \\
7.12688 \times 10^{11} \mathrm{i}\end{array}$ & $3.4421 \times 10^{9}$ & 1 & 2 & 3 \\
\hline$\omega_{7}$ & $\begin{array}{l}1.66551 \times 10^{15}- \\
1.0516 \times 10^{11} \mathrm{i}\end{array}$ & $\begin{array}{l}1.66545 \times 10^{15}- \\
1.06986 \times 10^{12} \mathrm{i}\end{array}$ & $5.64199 \times 10^{10}$ & 2 & 2 & 4 \\
\hline$\omega_{8}$ & $\begin{array}{l}1.73171 \times 10^{15}- \\
3.70973 \times 10^{12} \mathrm{i}\end{array}$ & $\begin{array}{l}1.73171 \times 10^{15}- \\
3.7085 \times 10^{12} \mathrm{i}\end{array}$ & $1.23369 \times 10^{9}$ & 1 & 2 & 3 \\
\hline$\omega_{9}$ & $\begin{array}{l}1.73768 \times 10^{15}- \\
2.4303 \times 10^{12} \mathrm{i}\end{array}$ & $\begin{array}{l}1.73768 \times 10^{15}- \\
2.43055 \times 10^{12} i\end{array}$ & $2.84178 \times 10^{10}$ & 1 & 2 & 3 \\
\hline$\omega_{10}$ & $\begin{array}{l}1.7766 \times 10^{15}- \\
2.79575 \times 10^{12} \mathrm{i}\end{array}$ & $\begin{array}{l}1.77659 \times 10^{15}- \\
2.79747 \times 10^{12} \mathrm{i}\end{array}$ & $6.09846 \times 10^{9}$ & 1 & 2 & 3 \\
\hline
\end{tabular}

\section{Comparison of the convergence rates of iterative methods}

Consider the question of the convergence rate of iterative methods (1.187) and (1.200) and Newton's method for solving the equation (1.182). First, we consider the case where we know quite good initial approximations of the poles. In this case, we apply these methods to improve estimates of the poles specified in column 2 of Table 1.2. Columns 5, 6, 7 in Table 1.2 show the number of iterations in the calculation of the poles by the methods of (1.200) (1.187) and with the use of Newton's method for solving the equation (1.182). From Table 1.2 it follows that the slowest convergence is observed for Newton's method in equation (1.182). The proposed method (1.200) and the method (1.187) require approximately the same number of iterations.

Let us now consider the convergence of iterative methods in more detail when the initial approximations of the poles are unknown. In this case, the following numerical experiment was carried out. A random point was selected from the considered frequency range and 
the iterative procedure was started. Tables $1.3-1.5$ give the average percentage of the initial approximations $\left(n_{\text {conv }}\right)$ converged to the pole, the average number of iterations $\left(n_{\text {iter }}\right)$, and the average number of calculations of the scattering matrix $\left(n_{\text {s-calc }}\right)$.

Note that various methods require different numbers of scattering matrix calculations at each iteration. For example, Newton's method and the method of (1.187) calculate the first derivative of the scattering matrix and require two calculations of the scattering matrix at each iteration, while Halley's method and the proposed method (1.199), (1.200) calculate the second derivative and require three scattering matrix computations per iteration. Therefore, when comparing the methods it is necessary to pay attention to the value $n_{\text {s-calc }}$ which is the total number of calculations of the scattering matrix. At the same time, at each iteration the scattering matrix can be calculated independently in parallel. Therefore, in parallel implementation of the iterative methods the value $n_{\text {iter }}$ is more important.

Table 1.3 was calculated for $N^{\prime}=21$. The table shows that among the scalar methods of solving the equations (1.182) and (1.183) fewer iterations are required when using Halley's method. This is because Halley's method has the order of convergence 3, while Newton'method has 2 [45]. Note also that Newton's method used for solving the equation (1.182) converges better than for the equation (1.183).

According to the number of calculations of the scattering matrix, the matrix methods (1.187), (1.199) and (1.200) have a better convergence compared with the scalar methods. The tables show that the proposed methods (1.199) and (1.200) require a smaller number of iterations of all the methods considered. In particular, the average number of iterations for the method (1.199) is almost 2 times smaller

Table 1.3. The convergence of iterative methods of the number of harmonics $N^{\prime}=$ $21(84 \times 84$ scattering matrix $){ }^{a}{ }^{a}-$ scalar method; ${ }^{b}$ - matrix method

\begin{tabular}{|c|c|c|c|c|}
\hline & $n_{\text {conv }}, \%$ & $n_{\text {iter }}$ & $n_{\text {s-calc }}$ & $n_{\text {near }}$ \\
\hline Newton's method for $(1.182)^{\mathrm{a}}$ & 98.5 & 9.85 & 19.7 & 57.2 \\
\hline Newton's method for $(1.183)^{\mathrm{a}}$ & 94.9 & 7,08 & 14,16 & 42.4 \\
\hline Halley's method for $(1.183)^{\mathrm{a}}$ & 99.9 & 4.20 & 12.60 & 54.5 \\
\hline Method $(1.187)^{\mathrm{b}}$ & 99.7 & 5.48 & 10.96 & 63.9 \\
\hline Method (1.199) ${ }^{\mathrm{b}}$ & 100 & 2.78 & 8.35 & 96.1 \\
\hline Method $(1.200)^{\mathrm{b}}$ & 100 & 3.40 & 10.2 & 64.6 \\
\hline
\end{tabular}


Table 1.4. The convergence of iterative methods of the number of harmonics $N^{\prime}=61(244 \times 244$ scattering matrix $)$

\begin{tabular}{lllll}
\hline & $n_{\text {conv }} \%$ & $n_{\text {iter }}$ & $n_{\text {s-calc }}$ & $n_{\text {near }}$ \\
\hline Newton's method for (1.182) & 82.5 & 11.79 & 23.58 & 37.7 \\
Newton's method for (1.183) & 87.2 & 7.1 & 14.2 & 24.5 \\
Halley's method for (1.183) & 99.7 & 4.54 & 13.62 & 40.3 \\
Method (1.187) & 78 & 5.95 & 11.9 & 48.6 \\
Method (1.199) & 100 & 2.88 & 8.64 & 87.6 \\
Method (1.200) & 100 & 3.72 & 11.16 & 43.75 \\
\hline
\end{tabular}

Table 1.5. The convergence of iterative methods of the number of harmonics $N^{\prime}=201(804 \times 904$ scattering matrix $)$

\begin{tabular}{llll}
\hline$n_{\text {conv }} \%$ & $n_{\text {iter }}$ & $n_{\text {s-calc }}$ & $n_{\text {near }}, \%$ \\
\hline 0 & - & - & - \\
94 & 6.83 & 13.66 & 26.5 \\
99 & 4.85 & 14.55 & 40 \\
0 & - & - & - \\
100 & 3.86 & 11.58 & 75.5 \\
100 & 3.94 & 11.82 & 45 \\
\hline
\end{tabular}

than for the method (1.187). At the same time, the average number of calculations for the scattering matrix method (1.199) is only 1.31 times smaller than for the method (1.187). Comparing the methods (1.199) and (1.200), we note that the method of (1.200), built on the assumption of the presence of a single resonance in the vicinity of the initial approximation of the pole, requires more iterations than the general method (1200), on average 1.22 times.

We now consider the methods at a higher value of the harmonics. Table 1.4 shows that when $N^{\prime}=61$ the convergence of the method (1.187) and the Newton method for equation (1.182) is deteriorating: the first method find the pole only in $78 \%$ of cases, the second is $83 \%$ of cases. If $N^{\prime}=201$ (Table 1.5), the method (1.187) and Newton's method for (1.182) stop working. Newton's and Halley's methods for the solution of equation (1.183) converge, but require about $30 \%$ more iterations (the number of calculations of the scattering matrix) than the proposed methods (1.199) and (1.200). Note that methods (1.199) and (1.200) converge in 100\% of cases.

In addition to the rate of convergence of another important characteristic of the iterative method is the shape of the attraction basin. Speaking about the calculation of the pole, the attraction basin 


\section{The Fourier modal method and its use in plasmonics and the theory of resonant diffraction gratings}

Moharam, M. G. , and T. K. Gaylord . "Rigorous coupled-wave analysis of planar-grating difaction." J. Opt. Soc. Am. 71, no. 7 (1981): 811-818.

Moharam, M. G. , and B. Eric . "Grann and Drew A. Pommet and T. K. Gaylord. Formu-lation for stable and effcient implementation of the rigorous coupled-wave analysis ofbinary gratings." J. Opt. Soc. Am. A 12, no. 5 (1995): 1068-1076.

Moharam, M. G. "Drew A. Pommet and Eric B. Grann and T. K. Gaylord. Stable implementation of the rigorous coupled-wave analysis for surface-relief gratings: enhanced transmittance matrix approach." J. Opt. Soc. Am. A 12, no. 5 (1995): 1077-1086.

Peng, S. , and G. M. Morris . "Effcient implementation of rigorous coupled-wave analysis for surface-relief gratings." J. Opt. Soc. Am. A 12, no. 5 (1995): 1087-1096.

$\mathrm{Li}$, Lifeng . "Use ofFourier series in the analysis of discontinuous periodic structures." J. Opt. Soc. Am. A 13, no. 9 (1996): 18701876.

Li, Lifeng . "New formulation of the Fourier modal method for crossed surface-relief gratings." J. Opt. Soc. Am. A 14, no. 10 (1997): 2758-2767.

Popov, E. , and M. Nevière . "Grating theory: new equations in Fourier space leading to fast converging results for TM polarization." J. Opt. Soc. Am. A 17, no. 10 (2000): 1773-1784.

$\mathrm{Li}$, Lifeng. "Fourier modal method for crossed anisotropic gratings with arbitrary per-mittivity and permeability tensors." J. Opt. A: Pure Appl. 5, no. 4 (2003): 345-355.

Popov, E. , and M. Nevière . "Maxwell equations in Fourier space: fast-converging formu-lation for difaction by arbitrary shaped, periodic, anisotropic media." J. Opt. Soc. Am. A 18, no. 11 (2001):

2886-2894.

Watanabe, K. , R. Petit , and M. Nevière . "Differential theory of gratings made of anisotro-pic materials." J. Opt. Soc. Am. A 19, no. 2 (2002): 325-334.

Zhou, C. , and L. Li . "Formulation ofthe Fourier modal method for symmetric crossed grat-ings in symmetric mountings." J. Opt. A: Pure Appl. Opt. 6 (2004): 4350.

Difactive computer optics. Ed. VA. Soifer, Moscow, Fizmatlit, 2007.

Methods for Computer Design of Difactive Optical Elements. Edited by Victor A, 159-266. New York, NY: Soifer. AWiley Interscience Publication. John Wiley \& Sons, 2002.

Gans, M. J. "A General Proof of Floquet's Theorem." IEEE Transactions on Micro-wave Theory and Techniques 13, no. 3 (1965): 384-385.

Electromagnetic Theory of Gratings: Topics in current physics (22, Ed. by R.Petit, N.Y: Springer-Verlag, 1980)

Višovský, S. , K. Postava , T. Yamaguchi, and R. Lopusník. "Magneto-Optic Ellipsometry in ExchangeCoupled Films." Appl. Opt. 41, no. 19 (2002): 3950-3960.

A. K. Zvezdin and V A. Kotov, Modern Magneto-Optics and Magneto-Optical Ma-terials, IOP Bristol, 1997. $\mathrm{Li}, \mathrm{L}$. "Formulation and comparison of two recursive matrix algorithms for modeling layered difaction gratings." J. Opt. Soc. Am. A 13, no. 5 (1996): 1024-1035.

T Vallius, J. Tervo, P Vahimaa, J. Turunen Electromagnetic feld computation in semiconductor laser resonators J. Opt. Soc. Am. A, 23(4), 906-911

Born, M. , and E. Wolf . Principles of optics. 4th ed. Pergamon Press, 1968.

Silberstein, E. , et al. "Use ofgrating theories in integrated optics." Journal ofthe Optical Society ofAmerica A. 18 , no. 11 (2001): 2865-2875.

Berenger J.-P A perfectly matched layer for the absorption of electromagnetic waves, Journal of Computational Physics. 1994. Vol. 114, No. 2. P. 185-200.

Hugonin, J. P. , and P. Lalanne. "Perfectly matched layers as nonlinear coordinate transforms: a generalized formalization." J. Opt. Soc. Am. A 22 (2005): 1844-1849.

Fan, S. , P. R. Villeneuve, J. D. Joannopoulos, and H. A. Haus . "Channel drop tunnel-ing through localized states." Phys. Rev. Lett. 80 (Feb. 1998): 960-963.

Suh, W. , and S. Fan . "Mechanically switchable photonic crystal flter with either all-pass transmission or fat-top refection characteristics." Opt. Lett. 28, no. 19 (Oct. 2003): 1763-1765.

Gippius, N. A. , and S. G. Tikhodeev . "The scattering matrix and optical properties ofmetamaterials." Phys. Usp. 52, no. 9 (2009): 1027-1030.

N. A. Gippius, T Weiss, S. G. Tikhodeev, and H. Giessen, "Resonant mode cou-pling of optical resonances in stacked nanostructures," Opt. Exp., vol. 18, no. 7, pp. 7569-7574, 2010.

Tikhodeev, S. G. , A. L. Yablonskii , E. A. Muljarov, N. A. Gippius, and T. Ishihara . "Quasiguided modes and optical properties ofphotonic crystal slabs." Phys. Rev. B 66, no. 4 (Jul. 2002): 045102.

D. A. Bykov and L. L. Doskolovich, "Magneto-optical resonances in periodic di-electric structures magnetized in plane," J. Mod. Opt., vol. 57, no. 17, pp. 1611-1618, 2010. 
Centeno, E. , and D. Felbacq . "Optical bistability in fnite-size nonlinear bidimen-sional photonic crystals doped by a microcavity." Phys. Rev. B 62 (Sep. 2000): R7683-R7686.

Centeno, E. , and D. Felbacq . "Rabi oscillations in bidimensional photonic crystals." Phys. Rev. B 62 (Oct. 2000): 10101-10108.

M. Nevière, E. Popov, and R. Reinisch, "Electromagnetic resonances in linear and nonlinear optics: Phenomenological study of grating behavior through the poles and zeros of the scattering operator," $J$. Opt. Soc. Amer. A, vol. 12, no. 3, pp. 513523, 1995.

M. Liscidini, D. Gerace, L. C. Andreani, and J. E. Sipe, "Scattering matrix analysis of periodically patterned multilayers with asymmetric unit cells and birefringentme-dia," Phys. Rev. B, vol. 77, Jan. 2008, Art. ID 035324.

D. Felbacq, "Numerical computation of resonance poles in scattering theory," Phys. Rev. E, vol. 64, Sep. 2001, Art. ID 047702.

T Weiss, N. A. Gippius, S. G. Tikhodeev, G. Granet, and H. Giessen, "Derivation of plasmonic resonances in the Fourier modal method with adaptive spatial resolution and matched coordinates," J. Opt. Soc. Amer. A, vol. 28, no. 2, pp. 238-244, Feb. 2011.

$\mathrm{Li}, \mathrm{L}$. "Field singularities at lossless metal-dielectric arbitrary-angle edges and their ramifcations to the numerical modeling of gratings." J. Opt. Soc. Amer. A 29, no. 4 (Apr. 2012): 593-604.

T Weiss, "Advanced numerical and semi-analytical scattering matrix calculations for modern nano-optics," Ph.D. dissertation, Fakultät Mathematik und Physik, Physikalisches Institut der Universität, Stuttgart, Germany, 2011.

Anemogiannis, E. , E. Glytsis, and T. Gaylord . "Effcient solution of eigenvalue equa-tions of optical waveguiding structures." J. Lightw. Technol. 12, no. 12 (Dec. 1994): 2080-2084.

Chen, C. , P. Berini , D. Feng, S. Tanev, and V. Tzolov . "Effcient and accurate numeri-cal analysis of multilayer planar optical waveguides in lossy anisotropic media." Opt. Exp. 7, no. 8 (Oct. 2000): 260-272.

Zolla, F. , G. Renversez, A. Nicolet, B. Kuhlmey, S. Guenneau, and D. Felbacq . Foun-dations of Photonic Crystal Fibres. London, U.K.: Imperial College, 2005.

D. Felbacq, "Finding resonance poles bymeans of cauchy integrals," in Proc. 13th Int. Conf. Transparent Optical Networks, Jun. 2011, paper We.B4.2.

V Lomakin and E. Michielssen, "Transmission of transient plane waves through perfect electrically conducting plates perforated by periodic arrays of subwave-length holes," IEEE Trans. Antennas Propag., vol. 54, no. 3, pp. 970-984, Mar. 2006.

A. Akimov, N. Gippius, and S. Tikhodeev, "Optical Fano resonances in photonic crystal slabs near difaction threshold anomalies," JETP Lett., vol. 93, pp. 427-430,

Gantmacher, F. R. The Theory of Matrices, 548. Moscow: Nauka, 1988.

Householder, A. S. The Numerical Treatment of a Single Nonlinear Equation, ser. Int. Series in Pure Appl. Math. New York: McGraw-Hill, 1970.

Bykov D. A., Doskolovich L. L. Numerical methods for calculating poles of the scattering matrix with applications in grating theory, J. Lightw. Technol. 2013.- March. Vol. 31, no. 5. pp. 793-801.

Hua, Y. , and T. K. Sarkar. "Matrix pencil and system poles." Signal Process. 21, no. 2 (1990): 195-198.

Hua, Y. , and T. K. Sarkar. "Matrix pencil method for estimating parameters of ex-ponentially

damped/undamped sinusoids in noise." IEEE Trans. Acoust., Speech, Signal Process. 38, no. 5 (May 1990): 814-824.

Hua, Y. , and T. K. Sarkar. "On SVD for estimating generalized eigenvalues of singu-lar matrix pencil in noise." IEEE Trans. Signal Process. 39, no. 4 (Apr. 1991): 892-900.

A.D.Rakic,A.B.Djurišic, J. M. Elazar, andM. L. Majewski, "Optical properties of metallic flms for verticalcavity optoelectronic devices," Appl. Opt., vol. 37, no. 22, pp. 5271-5283, Aug. 1998.

Yau, L. , and A. Ben-Israel . "The Newton and Halley methods for complex roots." Am. Math. Mon. 105, no. 9 (1998): 806-818.

Cao, Q. , P. Lalanne, and J.-P. Hugonin . "Stable and effcient Bloch-mode compu-tational method for onedimensional grating waveguides." J. Opt. Soc. Am. A 19 (2002): 335-338.

Bezus, E. A. , and L. L. Doskolovich . "Stable algorithm for the computation of the electromagnetic feld distribution of eigenmodes of periodic difaction structures." J. Opt. Soc. Am. A 29 (2012): 2307-2313.

Ebbesen, T. W. , H. J. Lezec , H. F. Ghaemi , T. Thio , and P. A. Wolff . "Extraordinary opti-cal

transmission through subwavelength hole arrays." Nature 391 (1998): 667-669.

Ghaemi, H. F. , T. Thio, and D. E. Grupp . "Surface plasmons enhance optical trans-mission through subwavelength holes." Phys. Rev. B 58 (1998): 6779-6782.

Le Perchec, J. , P. Qúemerais , A. Barbara , and T. Lopez-Rios . "Why metallic surfac-eswith grooves a few nanometers deep and wide may strongly absorb visible light." Phys. Rev. Lett. 100 (2008): 066408. Difactive nanophotonics. Edited by VA. Soifer, Moscow, Fizmatlit, 2011.

Belotelov, V. I. , D. A. Bykov, L. L. Doskolovich, A. N. Kalish, and A. K. Zvezdin . "Giant transversal Kerr effect in magneto-plasmonic heterostructures: The scatter-ing-matrix method." J. Exp. Theor. Phys. 110 (2010): 816-824. 
Belotelov, V. I. , D. A. Bykov , L. L. Doskolovich , A. N. Kalish , V. A. Kotov, and A. K. Zvez-din . "Giant magneto-optical orientational effect in plasmonic heterostructures." Optics Letters 34, no. 4 (2009): 398-400.

Sarrazin, M. , J.-P. Vigneron, and J.-M. Vigoureux . "Role of Wood anomalies in opti-cal properties of thin metallic flms with a bidimensional array of subwavelength holes." Phys. Rev. B 67 (2003): 085-415.

Vallius, T. , P. Vahimaa , and J. Turunen. "Pulse deformations at guided-mode resonance flters." Opt. Express 10 (2002): 840-843.

Vabishchevich, P. P. , V. O. Bessonov, F Yu Sychev, M. R. Shcherbakov, T. V. Dolgova, and A. A. Fedyanin . "Femtosecond Relaxation Dynamics of Surface PlasmonPolaritons in the Vicinity of Fano-Type Resonance." JETP Letters 92 (2010): 575-579.

Azaña, J. "Proposal of auniform fber Bragg grating as an ultrafast all-optical integra-tor." Optics Letters 33, no. 1 (2008): 4-6.

Preciado, M. A. , and M. A. Muriel . "Ultrafast all-optical integrator based on a fber Bragg grating: proposal and design." Optics Letters/Vol. 33, no. 12 (2008): 1348-1350.

Li, M. , D. Janner , J. Yao , and V. Pruneri . "Arbitrary-order all-fber temporal differentiator based on a fber Bragg grating: design and experimental demonstration." Opt. Ex-press 17, no. 22 (2009): 19798-807.

Berger, N. K. , B. Levit, B. Fischer, M. Kulishov, D. V. Plant, and J. Azaña . "Temporal dif-ferentiation of optical signals using aphase-shifted fber Bragg grating." Opt. Express 15, no. 2 (2007): 371-381.

Y Park, M. Kulishov, R. Slavík, J. Azaña. Picosecond and sub-picosecond fat-top pulse generation using uniform long-period fber gratings, Opt. Express 14(26), 12670-78 (2006).

Kulishov, M. , and J. Azaña . "Design of high-order all-optical temporal differentiators based on multiplephase-shifted fber Bragg gratings." Opt. Express 15, no. 10 (2007): 6152-6166.

Papoulis, A. The Fourier integral and its applications. New York, NY: McGraw-Hill, 1962.

Gippius, N. A. , S. G. Tikhodeev , and T. Ishihara . "Optical properties ofphotonic crys-tal slabs with an asymmetrical unit cell." Phys. Rev. B 72 (2005): 045-138.

Bykov, D. A. , L. L. Doskolovich, and V. A. Soifer ."Temporal differentiation of opti-cal signals using resonant gratings." Opt. Lett. 36 (2011): 3509-3511.

Bykov, D. A. , Capacity of resonant difactive gratings to differentiate the pulsed optical signa, Zh. Eksp.

Teor. Fiz., et al. "V 141." No. 5 (2012): 832839.

Abramowitz, M. , and I. A. Stegun . Handbook of Mathematical Functions. New York, NY: Dover

Publications, 1964.

D.A. Bykov, et al., Single-resonance difaction gratings for time-domain pulse transformations: integration of optical signals, J. Opt. Soc. Am. A. 2012.-August. Vol. 29, no. 8. Pp. 1734-1740.

D.A. Bykov, et al., Pis'ma Zh. Eksp. Teor. Fiz., 2012. V 95, No. 1. . 812.

Soifer V, Kotlyar V, Doskolovich L. Iterative Methods for Difactive Optical Ele-ments Computation.

Taylor\&Francis, 1997. 244 p.

Goodman J.W Introduction to Fourier Optics. New York: McGraw-Hill, 2005. 491 p.

Зверев В.А. Радіооптіка. Москва: Советское радіо, 1975. 304 с.

Raether H. Surface plasmons on smooth and rough surfaces and on gratings. Spring-er-Verlag, 1988. 136 p.

Jackson, J. D. Classical Electrodynamics. 3rd ed, 808. New York, NY: Wiley, 1998.

Johnson PB., Christy R.W Optical Constants of the Noble Metals, Physical Review B. 1972. Vol. 6, No. 12. P. 4370-4379.

Barnes, W. L. "Surface plasmonpolariton length scales: a route to sub-wavelength op-tics." Journal of Optics A: Pure and Applied Optics. 8, no. 4 (2006): S87-S93.

Berini P Long-range surface plasmon polaritons, Advances in Optics and Photonics. 2009. Vol. 1. P. 484-588.

Burke, J. , and G. Stegeman . "Tamir T Surface-polariton-like waves guided by thin, lossy metal flms." Physical Review B. 33, no. 8 (1986): 5186-5201.

Zia, R. , et al. "Geometries and materials for subwavelength surface plasmon modes." Journal ofthe Optical Society ofAmerica A. 21, no. 12 (2004): 2442-2446.

Avrutsky I. et al. Highly confned optical modes in nanoscale metal-dielectric mul-tilayers, Physical Review B. 2007. Vol. 75. P. 241402(R) (4pp).

Pollock, C. R. Fundamentals of Optoelectronics, 592. Chicago, IL: Irwin, 1995.

Kekatpure, R. D. , et al. "Solving dielectric and plasmonic waveguide dispersion rela-tions on a pocket calculator." Optics Express. 17, no. 26 (2009): 24112-24129.

Berini, P. "Plasmonpolariton modes guided by a metal flm of fnite width." Optics Letters. 24, no. 15 (1999): 1011-1013.

Holmgaard T., Bozhevolnyi S. Theoretical analysis of dielectric-loaded surface plas-mon-polariton waveguides, Physical Review B. 2007. Vol. 75, No. 24. P. 245405 (12 pp) . 
Kurihara, K. , and K. Suzuki . "Theoretical Understanding of an Absorption-Based Surface Plasmon Resonance Sensor Based on Kretchmann's Theory." Analytical Chemistry. American Chemical Society 74, no. 3 (2002): 696-701.

Bezus E.A., Doskolovich L.L., Kazanskiy N.L. Scattering suppression in plasmonic optics using a simple two-layer dielectric structure, Applied Physics Letters. 2011. Vol. 98, No. 22. P. 221-108 (3pp).

Bezus, E. A. , et al. "Pis'ma." Zh. Tekh. Fiz. V37, no. 23 (2011): 1018.

Bezus E.A., et al., Izv. RAN. Ser. Fiz., 2011. V 75, No. 12. C. 1674-1677.

Liu, Y. , et al. "Transformational Plasmon Optics." Nano Letters. 10, no. 6 (2010): 1991-1997.

Sannikov D.G., et al., Pis'ma Zh. Teor. Fiz., 2003. V 29, No. 9. 18.

Avrutsky, I. , and R. Soref . "Buchwald W Sub-wavelength plasmonic modes in a conduc-tor-gap-dielectric system with a nanoscale gap." Optics Express. 18, no. 1 (2010): 348-363.

Sámson, Z. L. , et al. "Femtosecond surface plasmon pulse propagation." Optics Letters. 36, no. 2 (2011): 250-252.

Zia R., Brongersma M.L. Surface plasmon polariton analogue to Young's double-slit experiment, Nature Nanotechnology. 2007. Vol. 2. P. 426-429.

Kim, H. , J. Hahn , and B. Lee . "Focusing properties of surface plasmon polariton foating dielectric lenses." Optics Express. 16, no. 5 (2008): 3049-3057.

Feng L. et al. Fourier plasmonics: Difactive focusing of in-plane surface plasmon polariton waves, Applied Physics Letters. 2007. Vol. 91, No. 8. P. 081-101 (3pp).

Bezus E.A. et al. Design of difactive lenses for focusing surface plasmons, Journal of Optics. 2010. Vol.

12, No. 1. P. 015-001 (7pp).

Bezus E.A. et al., Komp. optika, 2009. V 33, No. 2. 185-192.

Gramotnev, D. K. , and S. I. Bozhevolnyi . "Plasmonics beyond the difaction limit." Nature Photonics.

Nature Publishing Group 4, no. 2 (2010): 83-91.

Bai, B. , and L. Li . "Reduction of computation time for crossed-grating problems: a group-theoretic approach." Journal of the Optical Society ofAmerica A. 21, no. 10 (2004): 18861894.

Bai, B. , and L. Li . "Group-theoretic approach to enhancing the Fourier modal method for crossed gratings with square symmetry." Journal ofthe Optical Society ofAmerica A. 23, no. 3 (2006): 572-580.

Fikhtengol'ts G.M., Lectures in differential and integral calcules. Vol1, Moscow, Fizmatlit, 2001.

Bezus E.A. et al., Komp. Optika, 2008. V 32, No. 3. 234-237.

Bezus E.A. et al. Difaction gratings for generating varying-period interference pat-terns of surface plasmons, Journal of Optics A: Pure and Applied Optics. 2008. Vol. 10, No. 9. P. 095-204 (5pp).

Palik E.D. Handbook of Optical Constants. Academic press, 1985. Vol. 1. 294 p.

Doskolovich L.L., Kadomina E.A., Kadomin I.I. Nanoscale photolithography by means of surface plasmon interference, Journal of Optics A: Pure and Applied Op-tics. 2007. Vol. 9. P. 854-857.

Weber M.J. Handbook ofLaser Wavelengths. CRC Press, 1998. 784 p.

Bezus, E. A. , and L. L. Doskolovich . Komputernaya optika. 33, no. 1 (2009): 10-16.

Bezus, E. A. , and L. L. Doskolovich . "Grating-assisted generation of 2D surface plasmon interference patterns for nanoscale photolithography." Optics Communications. 283, no. 10 (2010): 2020-2025.

Belotelov VI. et al. Inverse Faraday effect in plasmonic heterostructures, Journal of Physics: Conference Series. 2010. Vol. 200, No. 9. P. 092-003 (4pp).

Bezus, E. A. , L. L. Doskolovich, and N. L. Kazanskiy . "Evanescent-wave interferometric nanoscale photolithography using guided-mode resonant gratings." Microelectronic Engineering. 88, no. 2 (2011): 170174.

Bezus, E. A. , L. L. Doskolovich , and N. L. Kazanskiy . "Interference pattern generation in evanescent electromagnetic waves for nanoscale lithography using waveguide dif-fraction gratings." Quantum Electronics. 41, no. 8 (2011): 759-764.

Brundrett, D. L. , et al. "Effects of modulation strength in guided-mode resonant sub-wavelength gratings at normal incidence, Journal of the Optical Society ofAmerica A." OSA 17, no. 7 (2000): 1221-1230.

Magnusson, R. , D. Shin , and Z. S. Liu . "Guided-mode resonance Brewster flter." Optics Letters. 23, no. 8 (1998): 612-614.

Tamir, T. , and S. Zhang . "Resonant scattering by multilayered dielectric gratings." Journal ofthe Optical Society ofAmerica A. 14, no. 7 (1997): 1607-1616.

Wei, C. , et al. "Electric feld enhancement in guided-mode resonance flters." Optics Letters. 31, no. 9 (2006): 1223-1225.

Sun T. et al. Electric feld distribution in resonant refection flters under normal incidence, Journal of Optics A: Pure and Applied Optics. 2008. Vol. 10. P. 125003 (5pp) .

Gippius, N. A. , and S. G. Tikhodeev . Usp. Fiz. Nauk 179 (2009): 1027-1030.

Belotelov, V. I. , et al. Zh. Eksper. Teor. Fiz. 137, no. 5 (2010): 932-942.

Bykov, D. A. , Zh Eksper, Teoret. Fiz., et al. "V 138." No. 6 (2010): 1093-1102. 
Blaikie, R. J. , and S. J. McNab . "Evanescent interferometric lithography." Applied Optics. 40, no. 10 (2001): 1692-1698.

Luo, X. "Ishihara T Surface plasmon resonant interference nanolithography tech-nique." Applied Physics Letters. 84, no. 23 (2004): 4780-4782.

Guo, X. , et al. "Large-area surface-plasmon polariton interference lithography." Optics Letters. 31, no. 17 (2006): 2613-2615.

Jiao $X$. et al. Numerical simulation of nanolithography with the subwavelength me-tallic grating waveguide structure, Optics Express. 2006. Vol. 14, No. 11. P. 4850-4860.

Handbook of Optics/ed. Bass M. New York: McGraw-Hill, 1995. Vol. 2. 1568 p.

Lifante G. Integrated photonics: fundamentals. Wiley, 2003. 184 p.

Belotelov, V. I. , et al. "Magnetooptical effects in the metal-dielectric gratings." Optics Communications. 278, no. 1 (2007): 104-109.

Madou M.J. Fundamentals ofmicrofabrication. CRC Press, 1997. 589 p.

Bezus E.A. et al. Komp. optika, 2011. V35, No. 4. 432-437.

\section{Components of photonic crystals}

J.D. Joannopoulos , S.G. Johonson , J.N. Winn , Photonic Crystals: Molding the Flow of Light, sec. ed., Princeton Univ. Press, 304p, 2008.

P.N. Prasad, Nanophotonics, Wiley, 432p, 2004.

A.Yariv , P. Yeh , Optical wave in crystals: propagation and control oflaser radiation, Wileylnterscience, 589p. (2002).

Y. Fukaya, D. Ohsaki, and T. Baba . Two-dimensional photonic cryctal waveguide with 600 bends in a thin slab structure. J. Jap. Soc. Appl. Phys. v. 39, no. 5A p. 2619-2623 (2000).

W. R. Frei , D. A. Tortorelli , H. T. Johnson . Geometry projection method for optimizing photonic nanostructures. Opt. Lett. 32, no. 1 pp. 77-79 2007.

Taflove A. Computational Electrodynamics: the finitedifference timedomain method. M.: Artech House, Inc. 1995.

Pernice W.H. , Payne F.P. , Gallagher D.F. . Numerical investigation of field enhancement by metal nano particles using a hybrid FDTDPSTD algorithm, Optics Express. -2007.Vol. 15.P. 1143311443.

Kim J.H. , Chrostowski L. , Bisaillon E. , Plant D.V. DBR, subwavelength grating, and photonic crystal slab FabryPerot cavity design using phase analysis by FDTD, Optics Express. -2007.Vol. 15.P. 1033010339. Yablonovitch E. Inhibited spontaneous emission in solidstate physics and electronics, Phys. Rev. - 1987. Vo1.58. P. 20592062.

Hugonin J.P. , Lalanne P. , White T.P. , Krauss T.F. Coupling into slowmode photonic crystal waveguides, Opt. Lett.2007.Vo1.32.P.26392640.

Kwan K.C. , Tao X.M. , Peng G.D. Transition of lasing modes in disordered active photonic crystals,Opt. Lett.2007.Vo1.32.P.27202722.

Zabelin V. , Dunbar L.A. , Thomas N.L. , Houndre R. , Kotlyar M.V. , O’Faolain L. , Krauss T.F. Self collimating photonic crystal polarization beam splitter, Opt. Lett.2007.Vo1.32.P.530532.

Li y. , Jin J. Fast fullwave analysis oflargescale threedimensional photonic crystal device, J. Opt. Soc. Am. B.2007.Vo1.24.P.24062415.

Mikaelyan A.L. , Dokl. AN SSSR, 1951, No. 81, 569571.

Snyder A.W. , Mitchell D.J. Spatial solitons ofthe powerlaw nonlinearity, Opt. Lett. -1993.Vo1.18.P.101

103.

Alimenkov I.V. , Komp. optika, 2005. No. 28. 4554.

Alimenkov I.V. , Komp. optika, 2005. No. 28. 5559.

Yee K. S. Numerical solution of initial boundary value problems involving Maxwell's equations in isotropic media, IEEE Trans. Antennas and Propagation. -1966. AP14. P.302307.

Moore G. Absorbing boundary conditions for the finitedifference approximation of the timedomain electromagnetic field equations, IEEE Trans. Electromagnetic Compatibility. -1981. Vo1.23. P. 377382. Berenger J. P. A perfectly matched layer for the absorption of electromagnetic waves, Computational Physics. -1994. Vol. 114. P. 185200.

y. Xu , R.K. Lee, A. Yariv , Adiabatic coupling between conventional dielectric waveguides with discrete translational symmetry, Opt. Lett., v.25, no. 10, p.755757 (2000).

A. Mekis , J. D. Joannopoulos . Tapered couplers for efficient interfacing between dielec-tric and photonic crystal waveguides. J. Light Techn. 19, no. 6 p. 861-865 (2001): .

T.D. Happ , M. Kamp , A. Forchel , Photonic crystal tapers for ultracompact mode conversion, Opt. Lett., v.26, p.14, p.11021104 (2001). 
A. Talneau , P. Lalanne, M. Agio , C. M. Soukoulis . Low-reflection photonic crystal taper for efficient coupling between guide sections of arbitrary widths. Opt. Lett. 27, no. 17 p/ 1522-1524 (2002).

V. R. Almeida, R. R. Panepucci, M. Lipson . Nanotaper for compact mode conversion. Opt. Lett. 28, no. 15 p. 1302-1304 (2003).

P. Bienstman , S. Assefa , S. G. Johson, J. D. Joannopoulos, G. S. Petrich , L. A. Koloziejski . Taper structures for coupling into photonic crystal slab waveguides. J. Opt. Soc. Am. B 20, no. 9 p. 1817-1821 (2003).

S. J. MacNab , N. Moll , Y. A. Vlasov . Ultra-low loss photonic integrated circit with membrane-type photonic crystal waveguide. Opt. Express 11, no. 22 p. 2927-2939 (2003).

P. E. Barclay , K. Srinivasan . 0. Painter, Design of photonic crystal waveguide for evanescent coupling to optical fiber tapers and integration with high-Q cavities. J. Opt. Soc. Am. B 20, no. 11 p. 2274-2284

(2003).

R. Orobtchouk , A. Layadi , H. Gualous , D. Pascal , A. Koster , S. Laval, Highefficiency light coupling in a submicrometric silicononinsulator waveguide, Appl. Opt., v.39, no.31, p.57735777 (2000).

S. Lardenois, D. Pascal , L. Vivien, E. Cassan , S. Laval , R. Orobtchouk, M. Heitzmann , N. Bonzaida , and L. Mollard .Low-loss submicrometer silicon-on-insulator rib wave-guides and corner mirrors. Opt. Lett. 28, no. 13 p. 1150-1153 (2003).

D. Taillaert , F. Vanlaere, M. Ayre , W. Bogaerts , D. VanThourhout , P. Bienstman , R. Baets . Grating couplers for couping between optical fiber and nanophotonic wave-guides. Jap. J. Appl. Phys. 45, no. 8 p. 6071-6077 (2006).

F. Van Laere G. Roelkens, M. Ayre, D.Taillaert, D. Van Thourhout , T.F. Krauss, R. Baets, Compact and high efficient grating couplers between optical fiber and nano-photonic waveguides, J. Light. Techn., v. 25, no. 1, p.151156 (2007).

B. L. Bachim , O. O. Ogunsola , and T. K. Gaylord . Optical fiber-to-waveguide coupling using carbondioxide-laser-induced long-period fiber gratings. Opt. Lett. 30, no. 16 p. 2080-2082 (2005).

D. W. Prather, J. Murakowski, S. Shi , S. Venkataraman, A. Sharkawy , C. Chen, D. Pustai . Highefficiency coupling structure for a single-line-defect photonic crystal waveguide.Opt. Lett. 27, no. 18 p. 1601-1603 (2002).

H. Kim , S. Lee, B.O.S. Park , E. Lee, High efficiency coupling technique for photonic crystal waveguides using a waveguide lens, OSA Techn. Digest: Frontiers in optics, 2003, MT68.

J. C. W. Corbett , J. R. Allington-Smith . Coupling starlight into single-mode photonic crystal fiber using a field lens Opt. Express 13, no. 17 p. 6527-6540 (2005).

D. Michaelis , C. Wachter , S. Burger , L. Zschiedrich , A. Brauer . Micro-optical assisted high-index waveguide coupling. Appl. Opt. 45, no. 8 1831-1838 (2006).

G. Kong , J. Kim , H. Choi , J. E. Im , B. Park, V. Paek, and B. H. Lee . Lensed photonic crystal fiber obtained by use of an arc discharge. Opt. lett. 31, no. 7 p. 894-896 (2006).

A. L. PokroOvsky , A. L. Efros . Lens based on the use of left-handed materials. Appl. Opt. 42, no. 28 p. 5701-5705 (2003).

N. Fabre, S. Fasquel, C. Legrand , X. Melique, M. Muller, M. Francois, O. Vanbesien, D. Lippens . Toward focusing using photonic crystal flat lens. Opto-electronics Review 14, no. 3 p. 225-232 (2006).

C. Li , M. Holt , A. L. Efros .Far-field imagimg by the Veselago lens made of a photonic crystal. J. Opt. Soc. Am. B 23, no. 3 p. 490-497 (2006).

T. Matsumoto , K. Eom , T. Baba . Focusing of light by negative refraction in a photonic crystal slab superlens on silicon-on-insulator substrate. Opt. Lett. 31, no. 18 p. 2786-2788 (2006).

C.Y. Li , J.M. Holt , A.L. Efros, Imaging by the Veselago lens based upon a twodimensional photonic crystal with a triangular lattice, J. Opt. Soc. Am. B, v.23, no.5, p.963968 (2006).

T. Geng , T. Lin , S. Zhuang .All angle negative refraction with the effective phase index of-I. Chinese Opt. lett. 5, no. 6p. 361-363 (2007).

T. Asatsume, T. Baba . "Abberation reduction and unique light focusing in a photonic crystal negative refractive lens." Opt. Express 16, no. 12 p. 8711-8718 2008.

N. Fabre , L. Lalonat , B. Cluzel , X. melique, D. Lippens , F. deFornel , O. Vanbesien « Measurement of a flat lens focusing in a 2D photonic crystal at optical wavelength», OSA Digest, CLEO/QELS, 2008,

CTuDD6, CA.

S. Yang , C. Hong , H. Yang . Focusing concave lens photonic crystals with magnetic ma-terials.J. Opt. Soc. Am. A 23, no. 4p. 956-959 (2006).

P. Luan, K. Chang , Photonic crystal lens coupler using negative refraction, Prog. In Electr. Res., v.3, no. I, p.9195 (2007).

S. Haxha, F. AbdelMalek. A novel design of photonic crystal lens based on negative re-fractive index.

Prog. In Electr. Res. 4, no. 2 p. 296-300 (2008).

Z. Lu, S. Shi , C. A. Schuetz, J. A. Murakowski, D. Prather . Three-dimensional pho-tonic crystal flat lens by full 3D negative refraction. Opt. Express 13, no. 15 p. 5592-5599 (2005). 
Z. Lu, S. Shi , C. A. Schuetz, D. W. Prather . Experimental demonstration of negative re-fraction imaging in both amplitude and phase. Opt. Express 13, no. 6 p. 2007-2012 (2005).

Minin, I. V. , O. V. Minin , Y. R. Triandafilov, and V. V. Kotlyar . Subwavelength diffiactive photonic crystal lens. Prog. In Electr. Res. B 7 257-264 (2008).

E. PshenaySeverin , C.C. Chen, T. Pertsch, M. Augustin , A. Chipoline , A. Tunnermann, Photonic crystal lens for photonic crystal waveguide coupling, OSA Techn. Digest:CLEO, 2006, CThK3.

Hugonin, J. P. , P. Lalanne, T. P. White, and T. F. Krauss . Coupling into clow-mode photonic crystal waveguide. Opt. Lett. 32, no. 18 p. 2638-2640 (2007).

Ya. R. Triandafilov, et al., Komp. optika, v.31, No. 3, 2731 (2007).

Y.R. Triandafilov, V.V. Kotlyar, Photonic crystal Mikaelian lens, Opt. Mem. Neur. Net., v.17, no. I, p. 17 (2008).

A.G. Nalimov , A.A. Kovalev , V. V. Kotlyar, V. A. Soifer , Threedimensional simulation of a nanophotonics device with use of fullwave software, Opt. Mem. Neur. Netw. (Inform.Opt.), v.18, no.2, pp.8592 (2009). Kotlyar, M. I. , Y. R. Traindaphilov, A. A. Kovalev, V. A. Soifer , M. V. Kotlyar , L. O'Faolain . Photonic crystal lens for coupling two waveguides. Appl. Opt. 48, no. 19 3722-3730 (2009).

Knight, J. C. , Birks T. A. , Russel P. S. J. , and Atkin D. M. . All-silica single mode optical fiber photonic crystal cladding. Opt. Lett. 1996 V. 21, No. 19 P. 1547-1549.

Adams M.J. An Introduction to Optical Waveguides. -New York, NY: Wiley, 1981.

Yeh P. , Yariv A. , Marom E. Theory of Bragg fiber, J. Opt. Soc. Am. 1978. V 68. P. 11961201.

Ibanescu M. , Fink M. , Fan S. , Thomas E.L. , Joannopoulos J.D. Alldielectric coaxial waveguide, Science. 2000. No. 289. P. 415419.

Cojocaru, E. Dispersion analysis of hollow-core modes in ultralarge-bandwith allsilica Bragg fibers, with nanosupports. Appl. Opt. 2006 V. 45, No. 9. P. 2039-2045.

Foroni M. et al., Confinement loss spectral behavior in hollowcore Bragg fiber, Opt. Lett. 2007. V 32. No. 21. P. 31643166.

Zhelticov, A. M. Ray-optic analysis of the (bio)sensing ability of ring-cladding hollow waveguides. Appl. Opt. 2008 47. No. 3 P. 474-479.

Dupuis A. et al., Guiding in the visible with, colorful solidcore Bragg fiber, Opt. Lett. 2007. V 32. No. 19. P. 28822884.

Fang Q. et al. Dispersion design ofallsolid photonic bandgap fiber, J. Opt. Soc. Am. A. 2007. V 24. No. 11. P. 28992905.

Ren, G. , et al. Low-loss all-solid photonic bangap fiber. Opt. Lett. 2007 V. 32. No. 9 P. 1023-1025.

Yang, R. , et al. Research of the effects of air hole shape on the properties of micro-structured optical fibers. Opt. Eng. 2004. V. 43, No. 11 P. 2701-2706.

Yue, Y. , et al. Highly birefringent elliptical-hole photonic crystal fiber with squeezed hexagonal lattice. Opt. Lett. 2007. V. 32, No. 5 P. 469-471.

Choi, H.-G. , et al. Discpersion and birefringence of irregularly microstructured fiber with elliptical core. Appl. Opt. 2007 V. 46, No. 35 P. 8493-8498.

Mafi A. , Moloney J.V. Shaping Modes in Multicore Photonic Crystal Fiber, IEEE Photonics Tech. Lett. 2005. V. 17. P. 348350.

Michaille, L. , et al. Characteristics of a Q-switched multicore photonic crystal fiber laser with a very large mode field area. Opt. Lett. 2008. V. 33, No. 171-73.

Szpulak M. et al. Experimental and theoretical investigations of birefringent holey fibers with a triple defect, Appl. Opt. 2005. V 44. P. 26522658.

Eguchi M. , Tsuji y Geometrical birefringence in squarelattice holey fibers having a core consisting ofmultiple defect, J. Opt. Soc Am. B. 2007. V. 24. No. 4. P.750755.

Zhang Ch. et al.Design of tunable bandgap guidance in highindex filled micro-structure fibers, J. Opt. Soc. Am. A. 2006. V 23. No. 4. P. 782786.

Sun J. et al. Refractive index measurement using photonic crystal fiber, Opt. Eng. 2007. V. 46. No. 1. P.014402.

Sun J. , Chan C.C. , Hybrid guiding in liquidcrystal photonic crystal fibers, J. Opt. Soc. Am. A. 2007. V 24. No. 10. P. 26402646.

Larsen, T. , et al. Optical devices based on liquid crystal photonic bandgap fibres. Opt. Express. $2003 \mathrm{~V}$. 11, No. 20 P. $2589-2596$.

Haakestad, M. W. , et al. Electrically tunable photonic bandgap guidance in a liquid-crystal-filled photonic crystal fiber. IEEE Photon. Technol. Lett. 2005 V. 17, No. 4 P. 819-821.

Domachuk, P. , Nguyen H. C. , Eggleton B. J. . Transverse probed microfluidic switch-able photonic crystal fiber devices. Photon. Technol. Lett. 2004 V. 16, No. 8 P. 1900-1902.

Ferrando A. et al. Nearly zero ultraflattened dispersion in photonic crystal fibers, Opt. Lett. 2000. V 25. P. 790792. 
Broderick A.N.G.R. Modeling large air fraction holey optical fiber, J. Opt. Tech. 2000. V. 18. P. 5056. Broderick A.N.G.R. et al. Nonlinearity in holey optical fibers: measurement and future opportunities, Opt. Lett. 1999. V. 24. P. 1395.

White, T. P. Multipole method for microstructured optical fibers. J. Opt. Soc. Am. A. 2002 V. 19, No. 10 P. 2322-2330.

Steel M.J. et al. Symmetry and degeneracy in microstructured optical fibers, Opt. Lett. 2001. V 26. P. 488 490.

Yamashita E. , Ozeki S. , Atsuki K. Modal analysis method for optical fibers with symmetrically distributed multiple cores, J. Lighhtwave Techn. 1985. V 3. P. 341-346.

G. Tayed, Scattering by a random set of parallel cylinders, G. Tayed et al., J. Opt. Soc. Am. A, 1994. Vol. 11. -PP. 25262538.

Sudbo, A. S. Film mode matching: A versatile method for mode field calculations in dielectric waveguides, A. S. Sudbo, Pure Appl. Opt. (J. Europ. Opt. Soc. A), 1993. Vol. 2. -PP. 211233.

Cucinotta, A. Holey fiber analysis through the finite element method, A. Cucinotta [and other], IEEE

Photon. Technol. Lett., 2002. Vol. 14. -PP. 15301532.

Brechet, F. Complete analysis of characteristics ofpropagation into photonic crystal fibers by the finite element method, F. Brechet [and other], Opt. Fiber Technol., 2000. Vol. 6(2). -PP. 181191.

Guan, N. Boundary Element Method for Analysis of Holey Optical Fibers, N. Guan [and other], J.

Lightwave Technol., 2003. Vol. 21(8).

Cheng, $\mathrm{H}$. Fast, accurate integral equation methods for the analysis ofphotonic crystal fibers, $\mathrm{H}$. Cheng [and other], Opt. Express, 2004.Vol. 12(16)-PP. 37913805.

Riishede, J. A Poor Man's Approach to Modeling MicroStructured Optical Fibers, J. Riishede, N.S. Mortensen and J. Legsgaard, J. Opt. A: Pure Appl. Opt., 2003. - Vol. 5. -PP. 534538.

Hardley, G.R. Fullvector waveguide modeling using an iterative finitedifference method with transparent boundary conditions, G.R. Hardley and R.E. Smith, J. Lightwave Technol., 1994. Vol. 13. -PP. 465469. Zhu, Z. Fullvectorial finitedifference analysis of microstructured optical fibers, Z. Zhu and T.G. Brown, Opt. Express, 2002. Vol. 10(17). -PP. 853864.

Jiang, W. An Extended FDTD Method With Inclusion of Material Dispersion for the FullVectorial Analysis of Photonic Crystal Fibers, W. Jiang [and other], J. Light-wave Technol., 2006. Vol. 24(11). -PP. 4417 4423.

$\mathrm{Xu}$, C.L. Fullvectorial mode calculations by finite difference method, C.L. Xu [and other], Inst Elec. Eng., Proc.J., 1994. Vol. 141. -PP. 281286.

Huang, W.P. The finitedifference vector beam propagation method. Analysis and Assessment, W.P. Huang [and other], J. Lightwave Technol., 1992. Vol. 10. -PP. 295305.

$\mathrm{Xu}, \mathrm{C} . \mathrm{L}$. Efficient and accurate vector mode calculations by beam propagation method, C.L. Xu , J. Lightwave Technol., 1993. Vol. 11(9). -PP. 12091215.

Itoh, T. Numerical techniques for microwave and millimeter-wave passive structures. T. Itoh-New York: Wiley, 1988.

Sorrentino, R. Transverse resonance technique, R. Sorrentino, Ch. 11 in Itoh's book [101].

Schlosser, W. Partially filled waveguides and surface waveguides of rectangular cross section. W.

Schlosser and H.G. Unger-New York: Advances in Microwaves -Academic Press, 1966.

Peng, S.T. Guidance and leakage properties of a class ofopen dielectric waveguides: Part IMathematical formulations, S.T. Peng and A.A. Oliner, IEEE Trans. Micro-wave Theory Techn., 1981. Vol. MTT29. -PP. 843-855.

Sudbo, A. S. Improved formulation ofthe film mode matching method for mode field calculations in dielectric waveguides, A.S. Sudbo , Pure Appl. Opt. (J. Europ. Opt. Soc. A), 1994. Vol. 3. -PP. 381-388. Kotlyar V.V. , Computer Optics, ISOI RAN, 2007. V. 31. -2730.

Ruhe, A. A Rational Krylov Algorithm For Nonlinear Matrix Eigenvalue Problems:/ A. Ruhe , Zapiski Nauchnih Seminarov, Steklov Mathematical Institute, 2000Vol. 268. PP. 176180.

Kotlyar V.V. , Computer Optics, ISOI RAN, 2005. V27. -89-94.

Kotlyar V.V. , Computer Optics, ISOI RAN, 2005. T. 27. -84-88.

Kotlyar V.V. , Computer Optics, ISOI RAN, 2003. T. 25. -41-48.

Jarlebring, E. , Rational Krylov for nonlinear eigenproblems, an iterative projection method, E. Jarlebring , H. Voss, Applications of Mathematics, 2005. Vo1.50, PP. 543-554.

Snaider, A. Theory of optical waveguids. Moscow: Radio i svyaz', 1987.

Kotlyar, V.V. Calculating spatial modes in photonic crystal fibers based on applying finite difference method to wave equations, V.V. Kotlyar, Y.O. Shuyupova, Proceedings of ICO Topical Meeting on Optoinformatics/Information Photonics 2006, September 47, 2006, St. Petersburg, Russia, PP. 483-485. Kotlyar V.V. , Computer Optics, ISOI RAN, 2005. V. 28. -41-44.

Kotlyar V.V. , Opticheskii zhurnal, 2007. -V74, No. 9. -600-608. 


\section{Sharp focusing of light with microoptics components}

Davidson, D.B. Body-of-revolution finite-difference time-domain modeling of space-time focusing by a three-dimensional lens, D.B. Davidson, R.W. Ziolkowski, J. Opt. Soc. Am. A. - 1994. - Vol. 11(4). - P 1471-1490.

$\mathrm{Yu}, \mathrm{W}$. On the solution of a class of large body problems with full or partial circular symmetry by using the finite-difference time-domain (FDTD) method, W. Yu, D. Arakaki, R. Mittra, IEEE Trans. on An. And Prop. - 2000. - Vol. 48(12). - P 18101817.

Farahat, N. A fast near-to-far-field transformation in body of revolution finite-difference time-domain method, N. Farahat, W. Yu, R. Mittra, IEEE Trans. on An. And Prop. - 2003.- Vol. 51(9). - P.2534-2540. Gedney, S.D. An anisotropic perfectly matched layer-absorbing medium for the trun-cation of FDTD lattices, S.D. Gedney, IEEE Trans. on An. And Prop. - 1996. - Vol. 44(12). - P 1630-1639.

Prather, D.W. Formulation and application of the finite-difference time-domain meth-od for the analysis of axially symmetric diffractive optical elements, D.W. Prather, S. Shi, J. Opt. Soc. Am. A. - 1999. - Vol. 16(5). - P 1131-1142.

Shi, S. Electromagnetic analysis of axially symmetric diffractive optical elements illu-minated by oblique incident plane waves, S. Shi, D.W. Prather, J. Opt. Soc. Am. A. - 2001. - Vol. 18(11). - P 2901-2907.

EL_Mashade, M.B. BOR-FDTD analysis of nonlinear Fiber Bragg grating and distributed Bragg resonator, M.B. EL_Mashade, M. Nady, Optics \& Laser Technology. - 2011. - Vol. 43(7). - P 1065-1072.

Antosiewicz, T.J. Dielectric-metal-dielectric nanotip for SNOM, T.J. Antosiewicz, P Wrobel, T. Szoplik, Proc. of SPIE. - 2009. - Vol. 7353.- P 73530I.

Olkkonen, J.T. On surface plasmon enhanced near-field transducers, J.T. Olkkonen, K. J. Kataja, J. Aikio, D.G. Howe, Proc. of SPIE. - 2004. - Vol.5380. - P 360-367.

Liu, Y. Analysis of a diffractive microlens using the finite-difference time-domain method, Y Liu, H. Liu, J. Micro/Nanolith. MEMS MOEMS. - 2010. - Vol. 9(3). - P 033004.

Liu, Y Broadband dispersion characteristics of diffractive microlenses based on the finite difference timedomain method, Y Liu, H. Liu, L. He, H. Zhou, C. Sui, Optics \& Laser Technology. - 2010. - Vol. 42(8). P 1286-1293.

Perez-Ocon, F. Fast Single-Mode Characterization of Optical Fiber by Finite-Difference Time-Domain Method, F. Perez-Ocon, A.M. Pozo, J.R. Jimenez, E. Hita, J. Lightwave Technol. - 2006. - Vol. 24(8). - P 3129.

Davidson, N. High-numerical-aperture focusing of radially polarized doughnut beams with a parabolic mirror and a flat diffractive lens, N. Davidson, N. Bokor, Opt. Lett. 2004.- Vol. 29(12). - P.1318-1320.

R. Borghi, M.Santarsiero, Nonparaxial propagation of spirally polarized optical beams, J. Opt. Soc. Am. A, v.21, no.10, p.2029-2037 (2004).

N. Passilly, R.S. Denis, K. Ait-Ameur, Simple interferometric technique for generation of a radially polarized light beam, J. Opt. Soc. Am. A, v.22, no.5, p.984-991 (2005).

T.G. Jabbour, S.M. Kuebler, Vector diffraction analysis of high numerical aperture focused beams modified by two- and three-zone annular multi-phase plates, Opt. Ex-press, v.14, no.3, p.1033-1043 (2006) .

Y Kozawa, S. Sato, Focusing property of a double-ring-shaped radially polarized beam, Opt. Lett., v.31, no.7, p.867-869 (2006).

Q. Zhan, Properties of circularly polarized vortex beams, Opt. Lett., v.31, no.7, p.867-869 (2006).

D. Deng, Nonparaxial propagation of radially polarized light beams, J. Opt. Soc. Am. B, v.23, no.6, p.12281234 (2006).

Y.I. Salamin, Fields of a radially polarized Gaussian laser beam beyond the paraxial approximation, Opt. Lett., v.31, no.17, p.2619-2621 (2006).

D. Deng, Q. Guo, L.Wu, X. Yang, Propagation of radially polarized elegant light beams, J. Opt. Soc. Am. B, v.24, no.3, p.636-643 (2007).

T. Grosjean, D. Courjon, C. Banier, Smallest lithographic marks generated by optical focusing systems, Opt. Lett., v.32, no.8, p.976-978 (2007).

Y Kozawa, S. Sato, Sharper focal spot formed by higher-order radially polarized laser beams, J. Opt. Soc. Am. A, v.24, no.6, p.1793-1798 (2007).

G.M. Lerman, U. Levy, Tight focusing of spatial variant vector optical fields with elliptical symmetry of linear polarization, Opt. Lett., v.32, no.15, p.2194-2196 (2007).

S. Yan, B. Yao, Description of a radially polarized Laguerre-Gauss beam beyond the paraxial approximation, Opt. Lett., v.32, no.22, pp.3367-3369 (2007).

E.Y.S. Yew. C.J.R. Sheppard, Tight focusing radially polarized Gaussian and Bessel- Gauss beams, Opt. Lett., v.32, no.23, p.3417-3419 (2007).

V. P. Kalosha, I. Golub, Toward the subdiffraction focusing limit of optical superresolution, v.32, no.24, p.3540-3542 (2007).

J. Stadler, C. Stanciu, C. Stupperich, A.J. Meixner „Tighter focusing with a parabolic mirror”, Opt. Lett., v.33, no.7, p.681-683 (2008). 
R. Dorn, S. Quabis, G. Leuchs, Sharper focus for a radially polarized light beam, Phys. Rev. Lett., v.91, p.233901 (2003).

Y Ohtaka, T. Ando, T. Inone, N. Matsumoto, H. Toyoda, Sidelobe reduction of tightly focused radially higher-order Laguerre-Gaussian beams using annular masks, Opt. Lett., v.33, no.6, p.617-619 (2008). C.J.R. Sheppard, M.A. Alonso, N.J. Moore, Lacalization measures for high-aperture wave fields based on pupil moments, J. Opt. A: Pure Appl. Opt.,v.10, p.033001 (2008).

A.P. Prudnikov, et al., Integrals and series: special functions. Nauka, Moscow, 1983.

Pohl, D.W. Optical spectroscopy: image recording with resolution L/20, D.W.Pohl, W.Denk, M.Lanz, Appl. Phys. Lett. - 1984. - Vol. 44. - P $651-653$.

Binnig, G. Tunneling through a controllable vacuum gap, G.Binnig, H.Rohrer, Ch.Gerber, E.Weibel, Appl. Phys. Lett. - 1982. - Vol. 40(2). - P 178.

McLeod, J.H. The Axicon: A New Type of Optical Element, J.H. McLeod, J. Opt. Soc. Am. - 1954. - Vol. 44(8). - P 592-597.

Dyson, J. Circular and Spiral Diffraction Gratings, J. Dyson, Proc. R. Soc. Lond. A. - 1958. - Vol. 248. - P 93-106.

Lit, J.W. Focal depth of a transmitting axicon, J.W. Lit, R. Tremblay, J. Opt. Soc. Am. - 1973.- Vol. 63, No. 4. - P 445-449.

Vahimaa, $P$ Electromagnetic analysis of nonparaxia I Bessel beams generated by diffractive axicon,

P.Vahimaa, V Kettunen, M.Knittinm, J.Turunen, J.Opt.Soc.Am. A. - 1997. - V4, -No. 8. - P 1817-1824.

Kizuka, T. Characteristics of a laser beam spot focused by a binary diffractive axicon, T. Kizuka, M.

Yamanchi, Y.Matsuoka, Opt.Eng. - 2008. - V.45. -No 5. - P.053401.

Kim, J.K. Compact all-fiber Bessel beam generator based on hollow optical fiber combined with a hybrid polymer fiber lens, J.K. Kim, J.Kim, YJung, W.Ha, YS.Jeong, S.Lee, A.Tunnermann, K.Oh, Opt.Lett. 2009. - V34, - No. 19. - P.2973-2975.

Kurt, H. Limited-diffraction light propagation with axicon-shape photonic crystal, H. Kurt, J. Opt. Soc. Am. B. - 2009. - Vol. 26. -No 5. - P 981-986.

Chen, W. Realization of an evanescent Bessel beam via surface Plasmon interference exited by a radially polarized beam, W. Chen, Q.Zhan, Opt. Lett. - 2009. - V 34. -No. 6. - P722-724.

Watanabe, K. Localized surface plasmon microscope with an illumination system employing a radially polarized zeros-order Bessel beam, K.Watanabe, G. Terakedo, H.Kano, Opt.Lett. - 2009. - vol.34, no.8. p.1180-1182.

Quabis, S. Focusing light to a tighter spot, S. Quabis, R. Dorn, M. Eberler, O. Glockl, G. Leuchs, Opt. Commun. - 2000. - Vol.179. - P.1-7.

Mote, R.G. Subwavelength focusing behavior of high numerical-aperture phase Fresnel zone plates under various polarization states, R.G. Mote, S.F. Yu, W. Zhou, X.F. Li, Appl. Phys. Let. - 2009. - Vol. 95. - P 191113.

Rajesh, K.B. Generation of sub-wavelength and super-resolution longitudinally polarized non-diffraction beam using lens axicon, K.B. Rajesh, P.M. Anbarasan, Chin. Opt. Lett. - 2008. - Vol. 6(10). - P 785-787. Wang, $\mathrm{H}$. Creation of a needle of longitudinally polarized light in vacuum using binary optics, $H$. Wang, L. Shi, B. Lukyanchuk, C. Sheppard, C.T. Chong, Nature photonics. - 2008. - Vol.2. - P 501-505.

Huang, K. Design of DOE for generating a needle of a strong longitudinally polarized field, K. Huang, $P$ Shi, X.-L. Kang, X. Zhang, Y-P Li, Opt. Lett. - 2010. - Vol. 35(7). - P 965-967.

Sun, C.-C. Ultrasmall focusing spot with a long depth of focus based on polarization and phase modulation, C.-C. Sun and C.-K. Liu, Opt. Lett. - 2003.- Vol. 28(2). - P 99-101.

Ohtake, Y. Sidelobe reduction of tightly focused radially higher-order Laguerre- Gaussian beams using annular masks, Y Ohtake, T. Ando, T. Inoue, N. Matsumoto, H. Toyoda, Opt. Lett. - 2008. - Vol. 33(6). P.617-619.

Youngworth, K.S. Focusing of high numerical aperture cylindrical vector beams, K.S. Youngworth, T.G. Brown, Opt. Expr. - 2000. - Vol. 7. - P 77-87.

Huang, K. Vector-vortex Bessel-Gauss beams and their tightly focusing properties, K. Huang, P Shi, G.W. Cao, K. Li, X.B. Zhang, Y.P Li, Opt. Lett. - 2011. - Vol. 36(6). - P 888-890.

Witkowska, A. All-fiber LP11 mode convertors, A. Witkowska, S. G. Leon-Saval, A. Pham, T. A. Birks, Opt. Lett. - 2008. - Vol. 33(4). - P.306-308.

Kozawa, Y Generation of a radially polarized laser beam by use of a conical Brewster prizm, Y Kozawa, S. Sato, Opt. Lett. - 2005. - Vol. 30(22). - P 3063-3065.

Golub, I. Characterization of a refractive logarithmic axicon, I. Golub, B. Chebi, D. Shaw, D. Nowacki, Opt. Lett. - 2010. - Vol. 35, No. 16. - P 2828-2830.

Golub, M.A. Focusing light into a specified volume by computer-synthesized hologram, M.A. Golub, S.V Karpeev, A.M. Prokhorov, I.N. Sisakyan, VA. Soifer, Sov. Tech. Phys. Lett. - 1981. - Vol. 7. - P 264-266.

Staronski, L.R. Lateral distribution and flow of energy in uniform-intensity axicon, L. R. Staronski, J.

Sochacki, Z. Jroszewicz, A. Kolodziejcziwz, J. Opt. Soc. Am. A. - 1992. - Vol. 9, No. 11. - P 2091-2094. 
Khonina S.N., et al., Komp. Optika, 2009. - V 33, No 4. - P 427-435.

Handbook of Mathematical Functions, edited by M. Ab-ramowitz, I.A. Stegun - Na-tional Bureau of Standards, Washington, DC, 1964.- 1044 p.

Kovalev A.A., et al., Komp. Optika, 2007. - V 31, No 4. - 29-32.

Kotlyar, V V Family of hypergeometric laser beams, V V Kotlyar, A.A. Kovalev, J. Opt. Soc. Am. A. - 2008. - Vol. 25. - P 262-270.

Prudnikov, A.P. Integrals and Series. Special functions, A.P. Prudnikov, Y.A. Brych- kov, O.I. Marichev. M.: Science, 1983.- (in Russian)

Snyder, A. Optical waveguide theory, A. Snyder, J. Love. - M.: Radio and Commu- ni-cations, 1987. - (in Russian)

Osterberg H. Closed solutions of Rayleigh's diffraction integral for axial points, H. Osterberg, L.Smith, J.Opt.Soc.Am. - 1961. - V51. - P.1050-1054.

Richards, B. Electromagnetic diffraction in optical systems II. Structure of the image field in an aplanatic system, B. Richards, E. Wolf, Proc. R. Soc. London A. - 1959. -Vol. 253.- P 358-379.

Debay, P Das Verhalten von Lichtwellen in der Nahe eines Brennpunktes oder einer Brennlinie, P Debay, Ann. d. Phys. - 1909. - Vol. 335, N 14. - P 755-776.

$\mathrm{Fu}, \mathrm{Y}$. Experimental investigation of superfocusing of plasmonic lens with chirped circular nanoslits, Y. Fu, Yu Liu, X. Zhou, Z. Xu, F. Fang, Opt. Exp. - 2010. - Vol. 18. - P 3438-3443.

$\mathrm{Fu}, \mathrm{Y}$. Plasmonic microzone plate: Superfocusing at visible regime, Y. Fu, W. Zhou, L.E.N. Lim, C.L. Du, X.G. Luo, Appl. Phys. Let. - 2007. - Vol. 91. - P 061124.

Mote, R.G. Near-field properties of zone plates in visible regime - New insights, R.G. Mote, S.F. Yu, B.K. Ng, W. Zhou, S.P Lau, Opt. Express. - 2008. - Vol. 16. - P 9554-9564.

Lopez, L.C. Vectorial diffraction analysis of near-field focusing of perfect black Fresnel zone plates under various polarization states, L.C. Lopez, M.P. Molina, P.A. Gon-zalez, S.B. Escarre, A.F. Gil, R.F. Madrigal, A.M. Cases, J. Light Technol. - 2011. - Vol. 29. -P 822-829.

$\mathrm{Fu}, \mathrm{Y}$. Hybrid Au-Ag subwavelength metallic structures with variant periods for superfocusing, Y. Fu, W. Zhou,J. Nanophotonics. - 2009. - Vol. 3.- P. 033504.

$\mathrm{Fu}, \mathrm{Y}$. Experimental study of plasmonic structures with variant periods for subwavelength focusing: analyses of characterization errors, Y. Fu, R.G. Mote, Q. Wang, W. Zhou, J. Mod. Opt. - 2009. - Vol. 56(14). - P 1550-1556.

Schonbrun, E. Scanning microscopy using a short-focallength Fresnel zone plate, E. Schonbrun, W.N. Ye, K.B. Crozier, Opt. Lett. - 2009. - Vol. 34(14). - P 2228-2230.

Novotny, L. Principles of nano-optics, L. Novotny, B. Hecht. - Cambridge University Press, 2006. -539 p. Michalski, K.A. Complex image method analysis of a plane wave-exited subwavelength circular aperture in a planar screen, K.A. Michalski, Prog. Electromag. Res. B. - 2011. - Vol. 27. - P 253-272.

$\mathrm{Wu}, \mathrm{J.H}$. Modeling of near-field optical diffraction from a subwavelength aperture in a thin conducting film, J.H. Wu, Opt. Lett. - 2011. - Vol. 36, N 17. - P 3440-3442.

Khonina, S.N. Controlling of the components of vectors of electrical and magnetic fields in a focus of lens with a high aperture with aim of binary phase structures, S.N. Khonina, S.G. Volotovsky, Computer optics, 2010. - V. 34. - No. 1. - PP. 58-68.

Lee, J.Y Near-field focusing and magnification through self-assembled nanoscale spherical lenses, J.Y. Lee, B.H. Hong, W.Y Kim, S.K. Min [and oth.], Nature, 2009. - V. 460,08173.- PP. 498-501.

Goldstein, D.J. Resolution in light microscopy studied by computer simulations, D.J. Godstein, J. Microsc., 1992. - V. 166. - PP. 185-197.

Bouhelier, A. Plasmon-coupled tip-enhanced near-field optical microscopy, A. Bouhelier, J. Renger, M.R. Beversluis, L. Novotny, J. Microsc., 2003.- V. 210. - PP. 220-224.

Karrai, K. Enhanced reflectivity contrast in confocal solid immersion lens microscopy, K. Karrai, X. Lorenz, Appl. Phys. Lett., 2000. - V. 77. - No. 21. - PP. 34593461.

Ippolito, S.B. High spatial resolution subsurface microscopy, S.B. Ippolito, B.B. Goldberg, M.S. Unlu, Appl. Phys. Lett., 2001. - V. 78. - No. 26. - PP. 4071-4073.

Koklu, F.H. Subsurface microscopy of integrated circuits with angular spectrum and polarization control,

F.H. Koklu, S.B. Ippolito, B.B. Goldberg, M.S. Unlu, Opt. Lett., 2009. - V. 34. - No. 8. - PP. 1261-1263.

Karabacak, D.M. Diffraction of evanescent waves and nanomechanical displacement detection, D.M.

Karabacak, K.L. Ekinci, C.H. Gan, G.J. Gbur [and oth.], Opt. Lett., 2007. - V. 32. - No. 13.- PP 18811883.

Mason, D.R. Enchanced resolution beyond the Abbe diffraction limit with wavelength-scale solid immersion lenses, D.R. Mason, M.V Jouravlev, K.S. Kim, Opt. Lett., 2010. - V. 35. - No. 12. - PP 20072009.

Zverev, V.A. Radio optics. M.: Sov. Radio, 1975. - 304 p.

Kotlyar, V. V. Subwave light localization in waveguide structures, V. V. Kotlyar, A.A. Kovalev, YO. Shujupova, A.G. Nalimov [and oth.], Computer optics, 2010. - V. 34. - No. 2. - PP. 169-186. 
Handmer, C.J. Blazing evanescent gtrating orders: a spectral approach to beating the Rayleigh limit, C.J. Handmer, C. Martijn de Sterke, R.C. McPhedran, L.S. Botten [and oth.], Opt. Lett., 2010. - V. 35. - No. 17. - PP. 2846-2848.

Thongrattanasiri, S. Analytical technique for subwavelength far field imaging, S. Thongrattanasiri, N.A. Kuhta, M.D. Escarra, A.J. Hoffman [and oth.], App. Phys. Lett., 2010. - V. 97. - PP 101103.

Pendry, J.B. Negative refraction makes a perfect lens, J.B. Pendry, Phys. Rev. Lett. 2000. - Vol. 85(18). P 3966-3969.

Blaikie, R.G. Imaging through planar silver lenses in the optical near field, R.G. Blaikie, D.O.S. Melville, J. Opt. A: Pure Appl. Opt. 2005. - Vol. 7(2). - P S176- S183.

Melvile, D.O.S. Super-resolution imaging through a planar silver layer, D.O.S. Mel- vile, R.J. Blaikie, Opt. Express 2005. - Vol. 13(6). - P 2127-2134.

Fang, N. Sub-diffraction-limited optical imaging with a silver superlens, N. Fang, H. Lee, C. Sun, X. Zhang, Science 2005. - Vol. 308(5721). - P 534-537.

Liu, Z. Far-field optical hyperlens magnifying sub-diffraction-limited object, Z. Liu, H. Lee, Y Xiong, C. Sun, X. Zhang, Science 2007. - Vol. 315(5819). - P 1686.

Thongrattanasiri, S. Hypergratings: nanophotonics in planar anisotropic metamaterials, S.

Thongrattanasiri, V.A. Podolskiy, Opt. Lett. 2009. - Vol. 34(7). - P 890-892.

Webb, K.J. Subwavelength imaging with a multilayer silver film structure, K.J. Webb, M. Yang, Opt. Lett. 2006. - Vol. 31(14). - P 2130-2132.

Liu, H. Webb. Submevelength imaging opportunities with planar uniaxial anisotropic lenses, H. Liu, Shivananad, K.J. Webb, Opt. Lett. 2008. - Vol. 33(21). - P 25682570.

Liu, H. Subwavelength imaging with nonmagnetic anisotropic bilayers, H. Liu, Shivanand, K.J. Webb, Opt. Lett. 2009. - Vol. 34(14). - P 2243-2245.

Wang, X. Unrestricted superlensing in a triangular two-dimensional photonic crystal, X.Wang, Z.F. Ren, K. Kempa, Opt. Express 2004. - Vol. 12(13). - P. 2919-2924.

Casse, B.D.F. Imaging with subwavelength resolution by a generalized superlens at infrared wavelengths, B.D.F. Casse, W.T. Lu, R.K. Banyal, Y.J. Huang, S. Sel- varasah, M.R. Dokmeci, C.H. Perry, S. Sridhar, Opt. Lett. 2009. - Vol. 34(13). - P 1994-1996.

Tsukerman, I. Superfocusing by nanoshells, I. Tsukerman, Opt. Lett. 2009. - Vol. 34(7). - P. 1057-1059. Ingrey, P.C. Perfect lens with not so perfect boundaries, P.C. Ingrey, K.I. Hopcraft, O. French, E. Jakeman, Opt. Lett. 2009. - Vol. 34(7). - P 1015-1017.

Ray, E.A. Simple demonstration of visible evanescent-wave enhancement with far- field detection, E.A. Ray,M. J. Hampton, R. Lopez, Opt. Lett. 2009. - Vol. 34(13). - P 2048-2050.

Cao, Z. Exact analytical method for planar optical waveguides with arbitrary index profile, Z. Cao, Y Jiang, Q. Shen, X. Dou, Y Chen, J. Opt. Soc. Am. A 1999. - Vol. 16(9). - P 2209-2212.

Chung, M. General eigenvalue equations for optical planar waveguides with arbitrarily graded-index profiles, M. Chung, C. Kim, J. Lightwave Techn. 2000. - Vol. 18(6). - P 878-885.

Born, M. Principles of optics, M. Born, E. Wolf - Moscow.: Nauka, 1973.- 719 p. - (in Russian).

Miller, W. Symmetry and Separation of Variables, W. Miller - Moscow.: Mir, 1981. - 342 p. - (in Russian). Korn, G. Mathematical handbook, G. Korn, T. Korn - Moscow.: Nauka, 1968. - 720 p. - (in Russian).

Triandafilov, YR. Photonic-crystal Mikaelian lens, YR. Triandafilov, V.V. Kotlyar, Computer Optics. - 2007. - V 31, N 3.- P 27-31. - ISSN 0134-2452. - (in Russian).

He, J. Wavelength switchable semiconductor laser using half-wave V-coupled cavities, J. He, D. Liu, Opt. Express 2008. - Vol. 16(6). - P 3896-3911.

Lin, X. Design and analysis of 2x2 half-wave waveguide couplers, X. Lin, D. Liu, J. He, Appl. Opt. 2009. Vol. 48(25). - P F18-F23.

Mikaelian, A.L. Application of stratified medium for waves focusing, A.L. Mikaelian, Doklady Akademii Nauk SSSR 1951. - Vol. 81. - P 569-571.

Kotlyar, V.V. Abel's transform in tasks of synthesis of gradient-index optical elements, V.V. Kotlyar, A.S. Melekhin, Computer Optics. - 2001. - N 22. - P 29-36. - ISSN 0134-2452. - (in Russian).

Kotlyar, V.V. Abel's transform for calculation of gradient-index optical elements with spherically-symmetric index distribution, V.V. Kotlyar, A.S. Melekhin, Computer Optics. - 2002. - N 24. - P 48-52. - ISSN 01342452. - (in Russian).

Kotlyar, V.V. Calculation of Maxwell's, Fish eye and Iton-Lipman's generalized lenses, V.V. Kotlyar, A.S. Melekhin, Computer Optics. - 2002. - N 24. - P 53-57. - ISSN 0134-2452. - (in Russian).

Alekseev, VM. Optimal control, VM. Alekseev, VM. Tikhomirov, S.V Fomin — Moscow.: Nauka, 1979 (in Russian).

Beliakov, G. Analysis of inhomogeneous optical systems by the use of ray tracing.I. Planar systems, G. Beliakov, D. Chan, Appl. Opt. 1997. - Vol. 36. - P 5303-5309.

Mikaelian, A.L. Self-focusing media with variable index of refraction, A.L. Mikae- lian, Prog. Opt. 1980. Vol. 17. - P 279-345. 
Rho, J. Spherical hyperlens for two-dimensional sub-diffractional imaging at visible frequencies, J. Rho, Z. Ye, Y Xiong, X. Yin, Z. Liu, H. Choi, G. Bartal, X. Zhang, Nature Communications 2010. - Vol. 1. - P 443. Kotlyar, V.V. et al., Komp. Optika 2012. - T. 36, N. 3.- C. 327-332.

Kotlyar, V V High resolution through graded-index microoptics, V V Kotlyar, A.A. Kovalev, A.G. Nalimov, S.S. Stafeev, Advances in Optical Technologies, 2012. - V 2012. - P 1-9.

Chien, H.T. Focusing of electromagnetic waves by periodic arrays of air holes with gradually varying radii, H.T. Chien and C.C. Chen, Opt. Exp. - 2006. - V 14. - P 10759.

Chen, Z. Photonic nanojet enhancement of backscattering of light by nanoparticles: a potential novel visible-light ultramicroscopy technique, Z. Chen, A. Taflove, V. Backman, Opt. Exp. - 2004. - v.12. p.1214.

$\mathrm{Li}, \mathrm{X}$. Optical analysis of nanoparticles via enhanced backscattering facilitated by 3-D photonic nanojets,

X. Li, Z. Chen, A. Taflove, V. Backman, Opt. Exp. - 2005. - v.13.- p.526.

Ferrand, P Direct imaging of photonic nanojets, P Ferrand, J.Wenger, A. Devilez, M. Pianta,B. Stout, N. Bonod, E. Popov, H. Rigneault, Opt. Exp. - 2008. - v.16. - p.6930.

Kim, M.-S. Engineering photonic nanojets, M.-S. Kim, T. Scharf, S. Muhlig, C. Rockstuhl, H.P. Herzig, Optics Express. - 2011. - v.19. - p.10206.

McCloskey, D. Low divergence photonic nanojets from Si3N4 microdisks, D. McCloskey, J.J. Wang, J.F. Donegan, Optics Express. - 2012. - Vol. 20. - p.128.

Kong, S.-C. Quasi one-dimensional light beam generated by a graded-index microsphere, S.-C. Kong, A. Taflove, V. Backman, Optics Express. - 2009. - v.17, p.3722.

Devilez, A. Three-dimensional subwavelength confinement of light with dielectric microspheres, A. Devilez, N. Bonod, J. Wenger, D. Gerard, B.S., H. Rigneault, E. Popov, Optics Express. - 2009. - v.17. - p.2089. Yi, K.J. Enhanced Raman scattering by self-assembled silica spherical microparticles, K.J. Yi, H. Wang, YF. Lu, Z.Y. Yang, J. Appl. Phys. - 2007. - v. 101. - p.063528.

Kong, S.-C. Photonic nanojet-enabled optical data storage, S.-C. Kong, A. Sahakian, A. Taflove, V. Backman, Opt. Exp. - 2008. - V16. - p.13713.

McLeod, E. Subwavelength direct-write nanopatterning using optically trapped microspheres, E. McLeod, C.B. Arnold, Nature Nano. - 2008. - v.3.- p.413.

Wang, T. Subwavelength focusing by a microsphere array, T. Wang, C. Kuang, X. Hao, X. Liu, J. Opt. 2011. - v.13. - p.035702.

\section{Focusing vortex beams and overcoming the diffraction limit}

BereznyiA, E. , et al. (1984). DAN SSSR , 234 (4), 802-805.

Khonina, S. N. , Kotlyar, V. V. , Shinkarev, M. V., Soifer, V. A. , \& Uspleniev, G. V. (1992). The rotor phase filter. J. Mod. Opt. , 39 (5), 1147-1154.

Diffiactibe computer optics (ed. V.A. Soifer), Moscow, Fizmatlit, 2007.

Prudnikov, A. P. , et al. (1983). Integrals and series. Special functions. Moscow: Nauka.

Handbook of mathematical function, ed by M. Abramovitz, I.A. Stegun NBS, Appl. Math. Ser. 55, 1964. Kotlyar V.V. , Almazov A.A. , Khonina S. N. , Soifer V.A. , Elfstrom H. and Turunen J. , Generation of phase singularity through diffiacting a plane or Gaussian beam by a spiral phase plate, J. Opt. Soc. Am. A, Vol. 22, No. 5, 849861 (2005)

Kotlyar, V. V. , Khonina, S. N. , Kovalev, A. A. , Soifer, V. A. , Elfstrom, H. , \& Turunen, J. (2006).

Diffraction of a plane, finite-radius wave by a spiral phase plate. Opt. Lett. , 31, 1597-1599.

Kotlyar, V. V. , Kovalev, A. A. , Khonina, S. N. , Skidanov, R. V. , Soifer, V. A. , Elfstrom, H. , Tossavainen, N. , \& Turunen, J. (2006). Diffiaction of conic and Gaussian beams by a spiral phase plate. Appl. Opt. , 45 (12), 2656-2665.

Khonina, S. N. , et al. (2010). Komp. Optika , 34 (3), 317-332.

Khonina, S. N. , et al. (2010). Izv. Samars. Nauch. Tsentra RAN , 12 (4), 15-25.

Karman, G.P. Airy pattern reorganization and subwavelength structure in a focus, G. P. Karman, M. W. Beijersbergen, A. van Duijl, D. Bouwmeester and J. P. Woerdman, J.Opt. Soc. Am. A. -1998. Vol. 15, No. 4. P. 884899.

Quabis, S. Focusing light to atighter spot, S. Quabis, R. Dorn, M. Eberler, O. Glockl and G. Leuchs, Opt. Commun. -2000. V.179. P.17.

13. Kant, R. Superresolution and increased depth of focus: an inverse problem of vector diffraction, J. Mod. Opt. -2000. -Vol. 47, N. 5. P. 905916.

Dorn, R. Sharper focus for a radially polarized light beam, R. Dorn, S. Quabis and G. Leuchs, Phys. Rev. Lett. -2003. V91. P.233901. 
Davidson, N. Highnumericalaperture focusing of radially polarized doughnut beams with a parabolic mirror and a flat diffiactive lens, N. Davidson, N. Bokor, Opt. Lett. -2004. -Vol. 29, No. 12. P. 13181320.

Sheppard, C.J.R. Annular pupils, radial polarization, and superresolution, C.J.R. Sheppard and A.

Choudhury, Appl. Opt. -2004. Vol. 43, No. 22. P. 43224327.

Pereira, S.F.Superresolution by means of polarisation, phase and amplitude pupil masks, S.F. Pereira, A. S. van de Nes, Opt. Commun. -2004. Vol. 234.P.119124.

Wang, H.Creation of a needle of longitudinally polarized light in vacuum using bi nary optics, H. Wang, L. Shi, B. Lukyanchuk, C. Sheppard and C.T. Chong, Nature Photonics. -2008. -Vol. 2. P. 501505.

Kozawa, y. Sharper focal spot formed by higherorder radially polarized laser beams, y Kozawa and $S$.

Sato, J. Opt. Soc. Am. A. -2007. V24. P.17931798.

Lerman, G.M. Effect of radial polarization and apodization on spot size under tight focusing conditions,

G.M. Lerman and U. Levy, Opt. Express. -2008. Vol. 16, No. 7. P. 45674581.

Kotlyar VV , et al., Komp. Optika, 2009. V33, No. 1. -5260

Zhan, Q. Cylindrical vector beams: from mathematical concepts to applications, Advances in Optics and Photonics. -2009. Vol. 1. P. 1-57.

Kozawa, y Generation of a radially polarized laser beam by use of a conical Brew ster prism, Yuichi Kozawa and Shunichi Sato, Opt. Lett. -2005. - V.30(22) . - P.30633065.

Nizfev V.G. , et al., Kvant. ELektronika.-2009. No. 39(6). P. 505514.

Bomzon, Z. Radially and azimuthally polarized beams generated by spacevariant dielectric subwavelength gratings, Z. Bomzon, G. Biener, V Kleiner, and E. Has man, Opt. Lett. -2002. - V27(5) . P.285287.

Yonezawa, K. Compact Laser with Radial Polarization Using Birefringent Laser Medium, Jpn., K.

Yonezawa, y. Kozawa, and S. Sato, J. Appl. Phys. -2007. - V.46(8A) . P. 51605163.

Tidwell, S.C. . Generating radially polarized beams interferometrically, S.C. Tidwell, D.H. Ford, and W.D. Kimura, Applied Optics. -1990. V29. P. 22342239.

Passilly, N. Simple interferometric technique for generation of a radially polarized light beam, Nicolas Passilly, Renaud de Saint Denis, and Kamel AitAmeur, Franqois Treussart, Rolland Hierle, and Jean Franqois Roch, J. Opt. Soc. Am. A. -2005. V.22(5). P. 984991.

Volpe, G. Generation of cylindrical vector beams with fewmode fibers excited by LaguerreGaussian beams, G. Volpe, D. Petrov, Opt. Comm. -2004. —V.237. P. 8995.

Davis, J.A. Two dimensional polarization encoding with a phase only liquid crystal spatial light modulator, J.A. Davis, D.E. McNamara, D.M. Cottrell, and T. Sonehara, Appl. Opt. -2000. V39. P. 154915541.

Neil, M.A.A. Method for the generation of arbitrary complex vector wave fronts, Mark A.A. Neil, Farnaz Massoumian, Rimvydas Juskaitis, and Tony Wilson, Opt. Lett. -2002. - V.27(21) . P. 19291931.

Iglesias, I. Polarization structuring for focal volume shaping in highresolution microscopy, Ignacio Iglesias, Brian Vohnsen, Opt. Commun. -2007. - Vol. 271. - P. 4047.

Khonina, S.N. Polarization converter for higherorder laser beams using a single binary diffiactive optical element as beam splitter, Khonina S.N., Karpeev S.V, Alferov S.V, Opt. Lett. -2012. Vol. 37, No. 12. P. 23852387

Cicchitelli, L. Longitudinal components for laser beams in vacuum, L. Cicchitelli, H. Hora, and R. Postle, Phys. Rev. A. -1990. Vol. 41. P. 37273732.

Simpson, N.B. Optical tweezers and optical spanners with LaguerreGaussian modes, N.B. Simpson, L. Allen, M.J. Padgett, J. Mod. Opt. -1996. Vol. 43(12). P. 24852491.

Heckenberg, N.R. Trapping microscopic particles with singular beams, N.R. Heckenberg, T.A. Nieminen, M.E. J Friese, H. RubinszteinDunlop, Proc. SPIE. —1998. Vol. 3487. P. 4653.

Helseth, L.E. Mesoscopic orbitals in strongly focused light, Opt. Commun. -2003. Vol. 224. P. 255261.

Soifer V.A. et al., Fizika Elementarnykh Chastits i Atomnogo Yadra. -2004. 35(6). C. 13681432.

Skidanov R.V., Komp. Optika. -2007. No. 31(1). P. 1421.

FrankeArnold, S. Advances in optical angular momentum, S. FrankeArnold, L. Allen, and M. Padgett, Laser Photonics Rev. -2008. Vol. 2. P. 299313.

Levenson, M.D. Optical vortex masks for via levels, M.D. Levenson, T. Ebihara, G. Dai, y. Morikawa, N. Hayashi, S.M. Tan, J. Microlith. Microfab. Microsys. -2004. Vol. 3 (2). P. 293304.

Unno, y Impact of mask errors and lens aberrations on the image formation by a vortex mask, $y$. Unno, $T$. Ebihara, M.D. Levenson, J. Microlith. Microfab. Micro sys. -2005. Vol. 4(2). P. 023006.

Willig, K.I. STED microscopy resolves nanoparticle assemblies, K.I. Willig, J. Kel ler, M. Bossi, S.W. Hell, New J. Phys. -2006. Vol. 8. P. 106.

Torok P. The use of GaussLaguerre vector beams in STED microscopy, P. Torok and P.R.T. Munro, Opt. Express. -2004. Vol. 12, No. 15. P. 36053617.

Helseth, L.E. Optical vortices in focal regions, Opt. Commun. -2004. Vol. 229. - P. 8591.

Liu, P. Phase singularities ofthe transverse field component ofhigh numerical aperture darkhollow

Gaussian beams in the focal region, P. Liu, B. Lu, Opt. Commun. -2007. Vol. 272. P. 18. 
Singh, R. K. Focusing of linearly, and circularly polarized Gaussian background vortex beams by a high numerical aperture system afflicted with thirdorder astigmatism, Rakesh Kumar Singh, P. Senthilkumaran, Kehar Singh, Opt. Commun. 2008. Vol. 281. P. 59395948.

48. Chen, B. Tight focusing of elliptically polarized vortex beams,, Baosuan Chen and Jixiong Pu, Appl. Opt. -2009. Vol. 48, No. 7. Pp. 12881294.

Rao, L. Focus shaping of cylindrically polarized vortex beams by a high numerical aperture lens, Lianzhou Rao, Jixiong Pu, Zhiyang Chen, Pu Yei, Opt. \&Las. Techn. -2009. Vol. 41. P. 241246.

Beth, R.A. Mechanical detection and measurement of the angular momentum of light, Phys. Rev. —1936. Vol. 50. P. 115125.

Holbourn, A.H.S. Angular momentum of circularly polarized light, Nature (Lon don). - 1936. Vol. 137. P. 31.

Allen, L. Orbital angular momentum of light and the transformation of Laguerre Gaussian laser modes, L. Allen, M. W. Beijersbergen, R. J. C. Spreeuw, and J. P. Woerdman, Phys. Rev. A. -1992. Vol. 45. P. 81858189.

Bamett, S.M. Orbital angularmomentum and nonparaxial lightbeams, S.M. Bar nett and L. Allen, Opt. Commun. -1994. Vol. 110. P. 670678.

Soskin, M.S. Topological charge and angular momentum of light beams carrying optical vortices, M. S.

Soskin, V.N. Gorshkov, M.V. Vasnetsov, J.T. Malos, and N.R. Heckenberg, Phys. Rev. A. -1997. Vol. 56. P. 40644075.

Richards, B. Electromagnetic diffraction in optical systems. II. Structure of the im age field in an aplanatic system, B. Richards and E. Wolf, Proc. Royal Soc. A. 1959. Vol. 253. P. 358379.

Prudnikov Yu.A. , et al., Integrals and series. Special functions. Moscow, Nauka, 1983.

Gori, F. Polarization basis for vortex beams, J. Opt. Soc. Am. A. -2001. Vol. 18, No. 7. P. 16121617.

Schwartz, C. Backscattered polarization patterns, optical vortices, and the angular momentum oflight, Chaim Schwartz and Aristide Dogariu, Opt. Lett. -2006.Vol. 31, No. 8. P. 11211123.

Bokor, N. A three dimensional dark focal spot uniformly surrounded by light, Nan dor Bokor, Nir Davidson, Opt. Commun. -2007. Vol. 279. P. 229234.

Grosjean, T. Photopolymers as vectorial sensors ofthe electric field, T. Grosjean and D. Courjon, Opt. Express. -2006. -Vol. 14, No. 6. P. 22032210.

Xie, X.S. Probing single molecule dynamics, X.S. Xie and R.C. Dunn, Science. 1994. Vol. 265. P. 361364. Beversluis, M.R. Programmable vector pointspread function engineering, M.R. Beversluis, L. Novotny, and S.J. Stranick, Opt. Express. -2006. —Vol. 14. —P. 26502656.

Khonina S.N. , et al., Komp. Optika. -2009. V.33, No. 4. C. 401411

Koronkevich, VP. Lensacon, VP . Koronkevich, I.A. Mikhaltsova, E.G. Churin, and Yu.I. Yurlov, Appl. Opt. -1993. -V 34(25). P. 57615772.

Kalosha, VP. Toward the subdiffiaction focusing limit of optical superresolution, V.P. Kalosha, I.Golub, Opt. Lett. -2007. V. 32. P. 35403542.

$\S$ Yirmiyahu, y . Excitation of a single hollow waveguide mode using inhomogeneous anisotropic subwavelength structures, Yaniv Yirmiyahu, Avi Niv, Gabriel Biener, Vladimir Kleiner, and Erez Hasman, Opt. Express. -2007. V. 15(20). P. 1340413414.

Khonina, S.N. Trochoson, S.N. Khonina, VV. Kotlyar, VA . Soifer, M.V. Shinkaryev, G.V. Uspleniev , Optics Communications. -1992. -V91(34). P. 158162.

Khonina, S.N. Rotation of microparticles with Bessel beams generated by diffiac tive elements, S.N. Khonina, V.V Kotlyar, R.V Skidanov, VA. Soifer, K. Jefimovs, 518 J. Simonen, J. Turunen, Journal of Modern Optics. -2004. -V 51(14). P. 21672184.

Zhan, Q. Properties of circularly polarized vortex beams, Opt. Lett. -2006. Vol. 31, No. 7. P. 867869. Khonina, S.N. Controlling the contribution ofthe electric field components to the fo cus of a highaperture lens using binary phase structures, Khonina S.N., Volotovsky S.G., J. Opt. Soc. Am. A. -2010. Vo1.27, No. 10. P. 21882197)

Goodman, G. (1970). Introduction into Fourier Optics. Moscow: Mir.

Durnin, J. Exact solutions for nondiffiacting beams. I. The scalar theory, J. Opt. Soc. Am. A. -1987. V. 4, N. 4. P. $651-654$

McLeod, J.H. The axicon: a new type of optical element, J. Opt. Soc. Am. -1954. - V. 44. P. 592597.

Fedotovsky, A. Optical filter design for annular imaging, Fedotovsky, A., Lehovec, H., Appl. Opt. —1974. Vol. 13(12). P. 29192923.

Khonina, S.N. Vortex phase transmission function as a factor to reduce the focal spot of highaperture focusing system, Khonina S. N., Kazanskiy N. L., Volotovsky S. G., Journal ofModern Optics. -2011. -V 58(9). P. 748-760

Khonina, S.N. Generation of rotating GaussLaguerre modes with binaryphase dif fractive optics, S.N. Khonina, VV Kotlyar, VA. Soifer, M. Honkanen, J. Lautanen, J. Turunen, Journal ofModern Optics. —1999. V. 46(2). P. 227238. 
Khonina, S.N. Encoded binary diffiactive element to form hypergeometric laser beams, S.N. Khonina, S.A. Balalayev, R.V. Skidanov, VV Kotlyar, B. Paivanranta, J. Turunen, J. Opt. A: Pure Appl. Opt. -2009. V. 11. P. 065702065709.

Toraldo di Francia, G. Supergain antennas and optical resolving power, G. Toraldo di Francia, Nuovo Cimento, Suppl. -1952. Vol. 9. P. 426.

Landberg, G. S. (2003). Optics. Moscow, Fizmatlit: Textbook.

Khonina S.N. , Komp. Optika2010. V 34(1). -3551

Khonina S.N. , et al.Komp. Optika2011. V. 35, No. 2. C. 203219.

Artl, J. Generation of a beam with a dark focus surrounded by regions of higher intensity: the optical bottle beam, J. Artl and M.J. Padgett, Opt. Lett. —2000. —V 25. P. 191193.

Hell, S.W. Breaking the diffraction resolution limit by stimulatedemissiondeple tion fluorescence microscopy, S.W. Hell and J. Wichmann, Opt. Lett. -1994. -V 19. P. 780782.

Biss, D.P. et al., Appl. Opt. -2006. -V45. P. 470479.

Khonina, S.N. Influence of vortex transmission phase function on intensity distribu tion in the focal area of highaperture focusing system, S.N. Khonina, N.L. Kazan skiy and S.G. Volotovsky, Optical Memory and Neural Networks (Allerton Press). -2011. V. 20(1). P. 2342.

Brent, R.P. Algorithms for Minimization Without derivatives, R.P. Brent. —Pren ticeHall, 1973. —195p.

87. http://alglib.sources.ru/

Sales, T.R.M.. Diffiactive superresolution elements, T.R.M. Sales and G.M. Morris, J. Opt. Soc. Am. A. -1997. Vol. 14. P. 1637.

Bewersdorf, J. 4piconfocal microscopy is coming of age, Joerg Bewersdorf, Alex ander Egner, Stefan W. Hell, G.I.T. Imaging \& Microscopy. -2004. Vol. 4. -P. 2425.

Helseth, L.E. Breaking the diffraction limit in nonlinear materials, L.E. Helseth, Opt. Commun. -2005. Vol. 256. P. 435.

Helseth, L.E. Smallest focal hole, Opt. Commun. -2006. V. 257. P. 18.

\section{Optical trapping and manipulation of micro-and nano-objects}

Ashkin, A. Acceleration and trapping of particles by radiation pressure. A. AshkinPhys. Rev. Lett. 24, no. 4 (1970): 156-159.

Ashkin, A. Observation ofresonances in the radiation pressure on dielectric spheres, A. Ashkin, J. M. Dziedzic, Phys. Rev. Lett. 38, no. 23 (1977): 1351-1354.

$\mathrm{He}, \mathrm{H}$. Direct observation of transfer of angular momentum to absorptive particles from alaser beam with a phase singularity. H. He, M. E. J. Friese, N. R. Heckenberg, H. Rubinsztein-Dunlop Phys. Rev. Lett. 75, no. 5 (1995): 826-829.

Sato, S. Optical trapping and rotational manipulation of microscopic particles and biological cells using higher-order mode Nd:YAG laser beams. S. Sato, M. Ishigure, H. Inaba, Electron. Lett. 27, no. 20 (1991): 1831-1832.

Bretenaker, F. Energy exchange between a rotating retardation plate and a laser beam, F Bretenaker, $\mathrm{A}$. Le Floch, Phys. Rev. Lett. -1990. -Vol. 65(18). -P. 2316.-->

Chen, $\mathrm{C}$. Analysis of vector Gaussian beam propagation and the validity of paraxial and spherical approximations. C. Chen, P Konkola, J. Ferrera, R. Keilmann, M. Schaffenberg Journal of Optical Society ofAmerica A. 19, no. 2 (2002): 404-412.

Cojoc, D. Design and fabrication of difactive optical elements for optical twee- zer arrays by means of ebeam lithography, D. Cojoc, E. Di Fabrizio, L.Businaro, S. Cabrini, F Romanato, L. Vaccari, M. Altissimo, Microelectronic Engineering. - 2002. -Vol. 61-62.-P. 963969.

Friese, M. E. J. Optical angular-momentum transfer to trapped absorbing particles. M. E. Friese, J. Enger, H. Rubinsztein-Dunlop, N. R. Heckenberg Phys. Rev. A. 54, no. 2 (1996): 1593-1596.

Khonina, S. N. The phase rotor flter. S. N. Khonina, V V Kotlyar, M. V Shinkaryev, G. V Uspleniev J.

Modern Optics. 39, no. 5 (1992): 1147-1154.

Volostnikov V.G. et al., , Izv. Samarsk. Nauchn. Tsentra RAN. -2000. -T2, N01. -C. 48-52.

Abramochkin, E. G. Microobject manipulations using laser beams with nonzero orbital angular momentum. E. G. Abramochkin, S. P Kotova, A. V Korobtsov, N. N. Losevsky, A. M. Mayorova, M. A. Rakhmatulin, V G. Volostnikov Laser physics 16, no. 5 (2006): 842-848.

Abramochkin, E. G. Application of spiral laser beams for beam shaping problem, E. G. Abramochkin, E. V Razueva, V G. Volostnikov, Proc. of LFNM. 29 June-1 July 2006, 275-278. Ukraine: Kharkiv, 2006.

Abramochkin, E. G. Fourier invariant singular wave-felds and beam shaping prob- lem, E. G.

Abramochkin, E. V Razueva, V G. Volostnikov, Proc. ofLFNM. -2006. -29 June- 1 July 2006, Kharkiv, Ukraine. -P. 370-373. 
Abramochkin, E. G. Gaussian beams: new aspects and applications, E. G. Abra- mochkin, V G. Volostnikov, Proc. of LFNM. -2006. -29 June - 1 July 2006, Kharkiv, Ukraine. -P. 267-274.

Paterson, L. Controlled rotation of optically trapped microscopic particles, L. Pa- terson, M. P MacDonald, J. Arlt, W Sibbett, P. E. Bryant, K. Dholakia, Science. -2001. -Vol. 292(5518). -P. 912-914.

Kotlyar VV, et al., Zh. Teor. Fiz., -1997. -V23, No. 17. -1-6.

Friese, M. E. J. Optical alignment and spinning of laser-trapped microscopic par- ticles, M. E. J. Friese, T. A. Nieminen, N. R. Heckerberg, H. Rubinsztein-Dunlop, Nature. -1998. -Vol. 394(6691). -P. 348-350.

Higurashi, E. Optically induced angular alignment of trapped birefringent microobjects by linear polarization, E. Higurashi, R. Sawada, T. Ito, Appl. Phys. Lett. -1998. -Vol. 73(21). -P. 3034-3036.

Holmlin, R. E. Light-driven microfabrication: Assembly of multicomponent, three- dimensional structures by using optical tweezers, R. E. Holmlin, M. Schiavoni, C. Y Chen, S. P Smith, M. G. Prentiss, G. M.

Whitesides, Angew. Chem. Int. Ed. Engl. -2000. -Vol. 39(19). -P3503-3506.

Grover, S. Automated single-cell sorting system based on optical trapping, S. C. Grover, A. G. Skirtach, R. C. Gauthier, C. P Grover, J. Biomed. Opt. -2001. -Vol. 6(1). -P. 14-22.

Fällman, E. Design for fully steerable dual-trap optical tweezers, E. Fällman, O. Axner, Appl. Opt. -1997. Vol. 36(10). -P. 2107-2113.

Sasaki, K. Pattern formation and fow control of fne particles by laser-scanning micromanipulation, K.

Sasaki, M. Kosioka, H. Misawa, N. Kitamura, H. Masuhara, Opt. Lett. -1991. -Vol. 16(19). -P. 1463-1465.

Dufresne, E. R. Optical tweezer arrays and optical substrates created with difactive optical elements, E. R.

Dufresne, D. G. Grier, Rev. Sci. Instr. -1998. -Vol. 69(5). -P. 1974-1977.

Dufresne, E. R. Computer-generated holographic optical tweezer arrays, E. R. Du- fresne, G. C. Spalding, M. T. Dearing, S. A. Sheets, D. G. Grier, Rev. Sci. Instrum. -2001. -Vol. 72(3). -P. 1810-1816.

Grier D. G., Dufresne E. R. Apparatus for applying optical gradient forces. US Pat- ent $6,055,106$. The University of Chicago. 2000.

Hahn, J. Real-time digital holographic beam-shaping system with a genetic feed- back tuning loop, J.

Hahn, H. Kim, K. Choi, B. Lee, Appl. Opt. -2006. -Vol. 45(5). -P. 915-924.

Curtis, J. E. Dynamic holographic optical tweezers, J. E. Curtis, B. A. Koss, D. G. Grier, Optics

Communications. -2002. -Vol. 207(1-6) .-P. 169175.

Reicherter, M. Optical particle trapping with computer-generated holograms written on a liquid-crystal display, M. Reicherter, T Haist, E. U. Wagemann, H. J. Tiziani, Opt. Lett. -1999. -Vol. 24 (9). -P. 608-610. Harris, M. Optical helices and spiral interference fringes, M. Harris, C.A. Hill, J. M. Vaughan, Optics Communications. -1994. -Vol. 106(4-6) .-P. 161-166.

Khonina S.N., et al., Komp. Optika-1999. -Bln. 19. -C.107-111.

Bandres, M. A. Parabolic nondifacting optical wave felds, M. A. Bandres, J. C. Gutierrez-Vega, S. ChavezCedra, Opt. Lett. -2004. -Vol. 29(1). -P. 44-46.

Abramochkin, E.G. Generalized Gaussian beams, E. G. Abramochkin, V G. Volost- nikov, J. Opt. A: Pure and Appl. Opt. -2004. -Vol. 6(5). -P. S157-S161.

Kotlyar, $\vee \vee$ Generation of phase singularity through difacting a plane or Gauss- ian beam by a spiral phase plate, V V Kotlyar, A. A. Almazov, S. N. Khonina, V A. Soifer, H. Elfstrom, J. Turunen, J. Opt. Soc. Am. A. -2005. -Vol. 22(5). -P. 849-861.

Bentley, J. B. Generation of helical Ince-Gaussian beams with a liquid-crystal dis- play, J. B. Bentley, J. A Devis, M. A. Bandres, J. C. Gutierrez-Vega, Opt. Lett. - 2006. -Vol. 31(5). -P. 649-651.

Chattrapiban, N. Generation of nondifacting Bessel beams by use of a spatial light modulator, N.

Chattrapaban, E. A. Rogers, D. Cofeld, W. T. Hill, R. Roy, Optics Let. -2003. -Vol. 28(22). -P. 2183-2185.

Kotlyar, $\mathrm{V} \vee$ Calculation of phase formers of non-difacting images and a set of concentric rings, $\mathrm{V} V$

Kotlyar, S. N. Khonina, V A. Soifer, Optik. - 1996. -Vol. 102(2). -P. 45-50.

Kotlyar, V V Rotation of multimodal Gauss-Laguerre light beans in free space and in a fber, V V Kotlyar, V A. Soifer, S. N. Khonina, Optics and Lasers in Engineering. -1998. -Vol. 29(4-5) . P. 343-350.

Rakhmatulin M.P, Development of methods of handling micro-objects by laser ra- diation. Dissertation,

Samara, 2003.

Ashkin, A. Observation of a single-beam gradient force optical trap for dielectric particles, A. Ashkin, J. M. Dziedzic, J. E. Bjorkholm, S. Chu, Optics Letters. -1986. -Vol. 11(5). -P. 288-290.

Ashkin, A. Optical levitation by radiation pressure, A. Ashkin, J. M. Dziedzic, Appl. Phys. Lett. - 1971. -Vol. 19(8). -P. 283-285.

Ashkin, A. Forces of a single-beam gradient laser trap on a dielectric sphere in the ray optics regime, $\mathrm{A}$.

Ashkin, Biophys J. - 1992. -Vol.61(2). -P. 569-582.

Ganic, D. Exact radiation trapping force calculation based on vectorial difaction theory, D. Ganic, X. Gan, M. Gu, Opt. Express. -2004. -Vol. 12(12). -P. 2670-2675.

Lemire, T. Coupled-multipole formulation for the threatment of electromagnetic scattery by a small dielectric particles of arbitrary sphere, T. Lemire, J.Opt.Soc.Am. A. -1997. -Vol. 14(2). -P. 470-474.

Marston, P. L. Radiation torque on a sphere caused by circulalarly-polarized elec- tromagnetic wave, P. L. Marston, J. H. Chrichton, Physical Review A. -1984. -Vol. 30(5). -P. 2508-2516. 
Navade, Y Radiation forces on a dielectric sphere in the Rayleigh scatterry regime, Y Navade, T. Asakure, Opt. Commun. -1996. -Vol. 124 (5-6). -P. 529-541.

Nieminen, T. A. Calculation and optical measurement of laser trapping forces on non-spherical particles, T. A. Nieminen, H. Rubinsztein-Dunlop, N. R. Heckenberg, Journal of Quantitative Spectroscopy \&Radiative Transfer. -2001. -Vol. 70(4-6)- P. 627637.

Rohrbach, A. Optical trapping of dielectric particles in arbitrary felds, A. Rohrbach, E. H. Stelzer, J. Opt. Soc. Am. A. -2001. -Vol. 18(4). -P. 813-835.

Pobre, R. Radiation force on a nonlinear microsphere by a lightly focused Gaussian beam, R. Pobre, C. Salome, Appl. Opt. -2002. -Vol. 41(36). -P. 7694-7701.

Debye, P Der Lichtdruck and Kugeln von beliebige Material, P Debye, Ann. Phys, 1909. -V 335(11). -P. 57-136.

Rockstuhl, C. Calculation of the torque on dielectric elliptical cylinders, C. Rock- stuhl, H. P Herzig, Opt. Soc. Am. A. -2005.-V. 22(1). -P. 109-116.

Shaohui, $Y$ Transverse trapping forces of focused Gaussian beam on ellipsoidal par- ticles, $Y$ Shaohui, $Y$ Baoli, J. Opt. Soc. Am. B. -2007. -Vol. 24(7). -P. 1596-1602.

Skidanov R.V, Komp. Optika-2005. -No. 28. -18-21.

Kotlyar VV, et al., Komp. Optika. -2005. -No. 27, -105-111.

Kotlyar VV, et al., Komp. Optika-2003. -Blп. 25. -24-28.

Kotlyar VV, et al., Opticheskii Zhurnal. -2005.-V. 72, No. 5. -C.55-61.

Arlt, J. Optical micromanipulation using a Bessel light beams, J. Arlt, V Garces- Chavez, W Sibbett, K. Dholakia, Opt. Comm. -2001. -Vol. 197. -P. 239-245.

Garces-Chavez, $\vee$ Simultaneous micromanipulation in multiple planes using a self- reconstructing light beam, V Garces-Chavez, D. McGloin, H. Melville, W Sibbett, K. Dholakia, Nature. -2002. -Vol. 419(6903). -P. 145-147.

MacDonald, M. P Creation and manipulation ofthree-dimensional optically trapped structures, M. P MacDonald, L. Paterson, K. Volke-Sepulveda, J. Arlt, W Sibbett, K. Dholakia, Science. -2002. -Vol. 296(5570). -P. 1101-1103.

Turunen, J. Holographic generation of difaction-free beams, J. Turunen, A. Vasara, A. T. Friberg, Applied Optics. -1988. -Vol. 27(19). -P. 3959-3962.

Soifer VA., et al., Komp. Optika. -2005. -Blп.28. -C.5-17.

Khonina, S.N. Rotating microobjects using a DOE-generated laser Bessel beam, S. N. Khonina, R. V Skidanov, V V Kotlyar, V A. Soifer, Proceedings of SPIE. -2004. -Vol. 5456.-P. 244-255.

Miller, W Symmetry and separation of variables, W Miller.-Cambridge University Press., 1977. -318p.

Allen, L. Orbital angular momentum of light and the transformation of Laguerre- Gaussian laser modes, $\mathrm{L}$. Allen, M. W Beijersbergen, R. J. C. Spreeuw, J. P Woerd- man, Phys. Rev. A. -1992. -Vol. 45(11). -P. 8185-8189.

Volke-Sepulveda, K. Orbital angular momentum of a high-order Bessel light beam, K. Volke-Sepulveda, V Garces-Chavez, S. Chavez-Cerda, J. Arlt, K. Dholakia, J. Opt. B: Quantum Semiclass. Opt. -2002. -Vol. 4(2). -P. S82-S89.

Durnin, J. Exact solution for nondifacting beams. I. The scalar theory, J. Durnin, J. Opt. Soc. Am. A. -1987. -Vol. 4(4). -P651-654.

Durnin, J. Difaction-free beams, J. Durnin, J. J. Miceli, J. H. Eberly, Phys. Rev. Lett. -1987. -Vol. 58(15). P. 14991501.

Paterson, C. Higher-order Bessel waves produced by axicon-type computer-gener- ated holograms, C.

Paterson, R. Smith, Optics Comm. -1996. -Vol. 124(1-2).-P. 121-130.

Fedotowsky, A. Optimal flter design for annular imaging, A. Fedotowsky, K. Leh- ovec, Appl. Opt. -1974. Vol. 13(12). -P. 2919-2923.

Skidanov R.V, Komp. Optika. -2006. -No. 29. -4-23.

Kotlyar VV, et al., Komp. Optika. -2005. -No.. 28. -29-36.

Soifer VA., et al. Proc. Second International Symposium Golografya EKSPO-2005. -69-70.

Khonina, S.N. DOE-generated laser beams with given orbital angular moment: ap- plication for micromanipulation, S. N. Khonina, R. V Skidanov, V V Kotlyar, V A. Soifer, J. Turunen, Proc. SPIE Int. Soc. Opt. Eng. -2005. -Vol. 5962. -P. 59622W

Khonina, S. N. Optical micromanipulation using DOEs matched with optical vorti- cies, S. N. Khonina, R. V Skidanov, V V Kotlyar, A. A. Kovalev, V A. Soifer, Proc. SPIE. -2006. -Vol. 6187. -P. 61871F Skidanov, R. V Optical microparticle trapping and rotating using multi-order DOE, R. V Skidanov, S. N. Khonina, V V Kotlyar, V A. Soifer, Proc. Ofthe ICO Topical Meeting on Optoinformatic/Information Photonics. -2006. -4-7 Sep. 2006, Saint- Peterburg, Russia. -P. 466-469.

Soifer, VA. Remarkable laser beams formed by computer-generated optical ele- ments: properties and applications, V A. Soifer, V V Kotlyar, S. N. Khonina, R. V Skidanov, Proc. SPIE. -2006. -Vol. 6252. -P. $62521 B$. 
Guo, C.-S. Optimal annulus structures of optical vortices, C.-S. Guo, X. Liu, J.-L. He, H. -T. Wang, Opt. Express. -2004. -Vol. 12(19). -P. 4625-4634.

Kotlyar, VV. Difaction of conic and Gaussian beams by a spiral phase plate, V V Kotlyar, A. A. Kovalev, S. N. Khonina, R. V Skidano, V A. Soifer, H. Elfstrom, N. Tossavainen, J. Turunen, Appl. Opt. -2006. -Vol. 45(12). -P2656-2665.

Soifer, VA. Optical data processing using DOEs, V A. Soifer, V V Kotlyar, S. N. Khonina, R. V Skidanov, Methods for Computer Design of Difactive Optical Ele- ments, Wiley-Interscience Publication John Wiley\& Sons, Inc, 2002. -Chapter 10. -P. 673-754.

Kotlyar, $\vee \vee$ Difaction of a fnite-radius plane wave and a Gaussian beam by a he- lical axicon and a spiral phase plate, V V Kotlyar, A. A. Kovalev, R. V Skidanov, O. Yu. Moiseev, V A. Soifer, J. Opt. Soc. Am. A. -2007. -Vol. 24(7). -P. 1955-1964.

Soifer VA., et al., Proc. All-Russian Semina: Yu.I. Desinyuk—founder of optical holography. FTI RAN, St. Petersburg. 2007-116-123.

Leach, J. Interactive approach to optical tweezers control, J. Leach, K. Wulff, G. Sinclair, P Jordan, J. Courtial, L. Thomson, G. Gibson, K. Karunwi, J. Cooper, Z. J. Laczik, M. Padgett, Appl. Opt. -2006. -Vol. 45(5). - P. 897903.

Xun, X. D. Phase calibration of spatially nonuniform spatial light modulators, X. D. Xun, R. W Cohn, Appl. Opt. -2004. -Vol. 43(35). -P. 64006406.

Kotlyar VV, et al., Komp. Optika. -2006. -No..30. - 16-22.

Kotlyar VV, et al., Komp. Optika. -2007.-V. 31, No. 1. -35-38.

Skidanov, R. V Micromanipulation in Higher-Order Bessel Beams, R. V Skidanov, $\vee \vee$ Kotlyar, $\vee \vee$ Khonina, A. V Volkov, V A. Soifer, Optical Memory and Neural Networks (Information Optics). -2007. -Vol. 16(2). -P. 91-98.

Davis, J.A. Difaction-free Beam Generated with Programmable Spatial Light Modulators, J. A. Davis, J.

Guertin, D. M. Cottrell, Appl. Opt. -1993. -Vol.32(31). -P. 6368-6370.

Davis, J. A. Intensity and phase measurements of nondifacting beams generated with the magneto-optic spatial light modulator, J. A. Davis, E. Carcole,D. M. Cot- trell, Appl. Opt. -1996. -Vol. 35(4). -P. 593-598. Skidanov VV., et al., Komp. Optika. -2007. V. 31, No. 1. -14-21.

Skidanov VV., et al., Izv. SNTs RAN. -2006.-V. 8, No. 4. -1200-1203.

Gao, M Generation and application ofthe twisted beam with orbital angular momen- tum, M. Gao, C. Gao, Z. L, Chinesse optics letters. -2007. -Vol. 5(2). -P. 89-92.

Farafonov, VS. A new solution of the light scattering problem for axisymmetric particles, $\vee \mathrm{S}$. Farafonov,

U. B. Ilin, T. A. Henning, Journal of Quantitative Spec- troscopy and Radiative Transfer. -1999. -Vol. 63(2-

6) .-P. 205-215.

Hong Du, Hao Zhang Ultra high precision Mie scattering calculations, 2002.

Jesacher, A. Fully phase and amplitude control of holographic optical tweezers with high effciency, A.

Jesacher, C. Maurer, A. Schwaighofer, S. Bernet, M. Ritsch- Marte, Optics Express. -2008. -Vol. 16(7). P4479-4486.

Montes-Usategui, M. Fast generation of holographic optical tweezers by random mask encoding of Fourier components, M. Montes-Usategui, E. Pleguezuelos, J, Andilla, E. Martin-Badosa, Optics Express. -2006. Vol.14(6). -P2101-2107.

Montes-Usategui, M. Adding functionalities to precomputed holograms with ran- dom mask multiplexing in holographic optical tweezers, M. Montes-Usategui, J. Mas, M. S. Roth, E. Martin-Badosa, Applied Optics. 2011. -Vol.50(10). -P. 1417-1424

Kotlyar VV, et al., Komp. Optika. -1999. -No. 19. -54-64.

Kotlyar VV, et al., Komp. Optika. -2001. -No. 21. -36-39.

Khonina, S.N. Encoded binary difactive element to form hyper-geometric laser beams, S.N. Khonina, S. A. Balalayev, R. V Skidanov, V V Kotlyar, B. Palvanranta, J. Tutunen, J. Opt. A: Pure Appl. Opt. -2009. -Vol. 11(6). -P. 065702-065708.

Khonina S.N., et al., Komp. Optika. -2009.-V. 33, No. 2. -138-146.

Cohn, R.W Approximating fully complex spatial modulation with pseudorandom phase-only modulation, R. W Cohn, M. Liang, Applied Optics. -1994. -Vol. 33(20). -P. 4406-4415.

Hassenbrook, L.G. Random phase encoding of composite fully complex flters, L. G. Hassenbrook, M. E.

Lhamon, R. C. Daley, R. W Cohn, M. Liang, Optics Letters. -1996. -Vol.21(4). -P. 272-274.

Skidanov R.V, et al., Komp. Optika. -2010. -V34, No. 2. -214-218.

Guo, C.-S. Optical sorting using an array of optical vortices with fractional topo-logical charge, C.-S. Guo, Y.-N. Yu, Zh. Hong, Optics Communications. -2010. - Vol.283(9). -P. 1889-1893.

Sinclair, G. Assembly of 3-dimensional structures using programmable holographic optical tweezers, G. Sinclair, P Jordan, J. Courtial, M. Padgett, J. Cooper, Z. Laczik, Optics Express. -2004. -Vol. 12(22). P5475-5480.

Polin, M. Optimized holographic optical traps, M. Polin, K. Ladavac, S.-H. Lee, Y. Roichman, D. G. Grier, Optics Express. -2005. -Vol.13(15). -P. 5831-5845. 
Abramochkin E.G., et al., Prod. 27th School for Coherent Optics and holography. Irkutsk-2005. 203-207. Cheong, F. C. Optical travelator: transport and dynamic sorting of colloidal micro- spheres with an asymmetrical line optical tweezers, F. C. Cheong, C. H. Sow, A. T. S. Wee, P Shao, A. A. Bettiol, J. A. van Kan, F Watt, Applied Physics B. -2006. - Vol.83(1). -P121-125.

Marchington, R.F Optical defection and sorting of microparticles in a near-feld optical geometry, R. F Marchington, M. Mazilu, S. Kuriakose, V Garces-Chavez, P. J. Reece, T. F Krauss, M. Gu, K. Dholakia, Optics Express. -2008. -Vol. 16(6). -P. 3712-3726.

Sun, YY Large-scale optical traps on a chip for optical sorting, Y. Y. Sun, X.-C. Yuan, L. S. Ong, J. Bu, S. W Zhu, R. Liu, Applied Physics Letters. -2007. -Vol. 90(3). -P. 031107-1-031107-3.

Rodrigo, P. J. Interactive light-driven and parallel manipulation of inhomogeneous particles, P. J. Rodrigo,

R. L. Eriksen, V R. Daria, J. Gluckstad, Optics Express. - 2002. -Vol. 10(26). -P. 1550-1556.

Dasgupta, R. Microfuidic sorting with a moving array of optical traps, R. Dasgupta, S. Ahlawat, P. K.

Gupta, Applied Optics. -2012. -Vol. 51(19). -P. 4377-4387.

Rodrigo, P. J. Dynamic formation of optically trapped microstructure arrays for bio- sensor applications, $P$. J. Rodrigo, R. L. Eriksen, V R. Daria, J. Gluckstad, Biosen- sors and Bioelectronics. -2004. -Vol. 19(11). P. 1439-1444.

Rodrigo, P. J. Real-time interactive optical micromanipulation of a mixture of high- and low-index particles, P. J. Rodrigo, V R. Daria, J. Gluckstad, Optics Express. -2004. -Vol. 12(7) -P1417-1425.

Prentice, P. A. Manipulation and fltration of low-index particles with holographic Laguerre-Gaussian optical trap arrays, P. A. Prentice, M. P MacDonald, T. G. Frank, A. Cuschieri, G. C. Spalding, W Sibbett, P. A. Campbell, K. Dholakia, Optics Ex- press. -2004. -Vol.12(4). -P. 593-600.

Porfr'ev A.P, Vest. Samarsk. Gosud. Aerokosmich. Univ., -2010. -No. 4(24). - 269-275.

Balalaev S.A., et al., Komp. Optika. -2007. -V31, No. 4. -23-29.

Herman, R.M. Hollow beams of simple polarization for trapping and storing atoms, R. M. Herman, T. A. Wiggins, J. Opt. Soc. Am. -2002. -Vol. 19(1). -P. 116-121.

McQueen, C. A. An experiment to study a"nondifacting" light beam, C. A. Mc- Queen, J. Arlt, K. Dholakia, Am. J. Phys. -1999. -Vol. 67(10). -P. 912915.

Khonina, S.N. An analysis of the angular momentum of a light feld in terms of angular harmonics, S. N. Khonina, V V Kotlyar, V A. Soifer, P Paakkonen, J. Sim- onen, J. Turunen, Journal of Modern optics. -2001. -Vol. 48(10). -P. 1543-1557.

Khonina, S. N. Rotation of microparticles with Bessel beams generated by difac- tive elements, S. N. Khonina, V V Kotlyar, R. V Skidanov, V A. Soifer, K. Je- fmovs, J. Simonen, J. Turunen, Journal of Modern Optics. -2004. -Vol. 51(14). -P2167-2184.

Kotlyar, VV An algorithm for the generation of laser beams with longitudinal periodicity: rotating images, $\mathrm{V}$ V Kotlyar, S. N. Khonina, V A. Soifer, Journal of Modern Optics. -1997. -Vol. 44(7). -P. 1409-1416.

Paakkonen, P Rotating optical felds: experimental demonstration with difactive optics, P Paakkonen, J. Lautanen, M. Honkanen, M. Kuittinen, J. Turunen, S. N. Khonina, V V Kotlyar, V A. Soifer, A. T. Friberg, Journal ofModern Optics. - 1998. -Vol. 45(11). -P. 2355-2369.

Garces-Chavez, $\vee$ Simultaneous micromanipulation in multiple planes using a self- reconstructing light beam, V Garces-Chavez, D. McGloin, H. Melville, W Sibbett, K. Dholakia, Nature. -2002. -Vol. 419(6903). -P. 145-147.

Porfrev, A. P The generation of the hollow optical beams array by the superpo- sitions of Bessel beams, A. P Porfrev, Asia-Pacifc Conference on Fundamental Problems of Opto-and Microelectronics (Russia, Moscow-Samara, 4-8 July). -LPI, 2011. -1 DVD-ROM, SAMP15.

Khonina, S. N. Astigmatic Bessel laser beams, S. N. Khonina, V V Kotlyar, V A. Soifer, K. Jefmovs, P Paakkonen, J. Turunen, Journal of Modern Optics. -2004. - Vol. 51(5). -P677-686.

Chavez-Cerda, S. Interference of traveling nondifacting beams, S. Chavez-Cer- da, M. A. Meneses-Nava, J. M. Hickmann, Optics Letters. - 1998. -Vol. 23(24). -P1871-1873.

Dudley, A. Superpositions of higher-order Bessel beams and nondifacting speckle felds, A. Dudley, R. Vasilyeu, A. Forbes, N. Khilo, P Ropot, V Belyi, N. Kazak, Proc. of SPIE. -2009. -Vol. 7430. -P.74300A-1 74300A-7.

McGloin, D. Interfering Bessel beams for optical micromanipulation, D. McGloin, V Garces-Chavez, K. Dholakia, Optics Letters. -2003. -Vol. 28(8). -P657-659.

Vasilyeu, R. Generating superpositions of higherorder Bessel beams, R. Vasilyeu, A. Dudley, N. Khilo, A. Forbes,Optics Express. -2009. -Vol. 17(26). -P. 23389-23395.

Difactive computer optics. VA. Soifer (editor), Moscow, Fizmatlit, 2007.

Khonina, S. N. Generating a couple of rotating nondiffarcting beams using abinary- phase DOE, S. N. Khonina, V V Kotlyar, V A. Soifer, J. Lautanen, M. Honkanen, J. Turunen, Optik. - 1999. -Vol. 110(3). -P 137-144.

Kotlyar VV, et al., Avtometriya. - 1997. -No. 5. -46-54.

Doskolovich, L. L. Analytical initial approximation for multiorder binary gratings design, L. L. Doskolovich, V A. Soifer, G. Alessandretti, P Perlo, M. Repetto, Pure and Appl.Opt.- 1994. -Vol.3(6). -P921-930. 
Krackhardt, U. Upper bound on the difaction effciency of phase-only fanout ele- ments, U. Krackhardt, J. N. Mait, N. Streibl, Applied Optics. - 1992. -Vol. 31(1). -P27-37.

Zhou, C. Numerical study of Dammann array illuminators, C. Zhou, L. Linen, Ap- plied Optics. -1995. -Vol. 34(26). -P5961-5969.

Kotlyar VV, et al., Komp. Optika. -1999. -No. 19. -74-79.

Doskolovich L.L., et al., Komp. Optika. - 1996. -No.16. -31-35.

Khonina, S. N. Amethod for design of composite DOEs for the generation of letter image, S. N. Khonina, V V Kotlyar, V V Lushpin, V A. Soifer, Optical Memory and Neural Networks. - 1997. -Vol. 6(3). -P213-220.

Kotlyar VV, et al., Komp. Optika. - 1996. -No. 16. -50-53.

Gluckstad, J. Dynamic array of dark optical traps, J. Gluckstad, V R. Daria, P. J. Rodrigo, Applied Physics Letters. -2004. -Vol. 84(3). -P. 323-325.

Yuan, X.-C. The generation of an array of nondifacting beams by a single compos- ite computer generated hologram, X.-C.Yuan, S. H. Tao, B. S. Ahluwalia, J.Opt.A.:Pure Appl.Opt. - 2005. -Vol.7(1). -P40-46.

Skidanov R.V, et al., Komp. Optika. -2012. -V36, No. 1. -80-90.

Skidanov R.V, et al., Komp. Optika. -2012. -V36, No. 2. -211-218.

Leitz, G. Stress response in Caenorhabditis elegans caused by optical tweezers: wavelength, power, and time dependence, G. Leitz, E. Fällman, S. Tuck, O. Axner, Biophys. J. -2002-Vol. 82(4). -P. 2224-2231. König, K. Cell damage in near-infrared multimode optical traps as a result of mul- tiphoton absorption, K. König, H. Liang, M.W Berns, B.J. Tromberg, Opt Lett. - 1996. -Vol. 21(14). -P. 10901092.

Thanh, S.D. Photogenerated singlet oxygen damages cells in optical traps, S.D. Thanh, N.C. Zakharov, arXiv:0911.4651-2009.

Peterman, E.J.G. Laser-Induced Heating in Optical Traps, E.J.G. Peterman, F. Gittes, C.F Schmidt, Biophysical Journal. -2003. -Vol. 84(2). -P. 13081316.

Mirsaidov, U. Optimal optical trap for bacterial viability, U. Mirsaidov, W Timp, K. Timp, M. Mir, P Matsudaira, G. Timp, Phys Rev E Stat Nonlin Soft Matter Phys. - 2008. -Vol. 78(2 Pt 1). -P. 021910. van der Horst, A. Calibration of dynamic holographic optical tweezers for force measurements on biomaterials, A. van der Horst, N.R. Forde, Opt Express. -2008. - Vol. 16(25). -P. 20987-21003.

Emiliani, $\vee$ Wave front engineering for microscopy of living cells, V Emiliani, D. Cojoc, E. Ferrari, $\vee$ Garbin, C. Durieux, M. Coppey-Moisan, E. Di Fabrizio, Opt Express. - 2005. -Vol. 13(5). -P. 1395-1405.

Xin, $\mathrm{H}$. Targeted delivery and controllable release of nanoparticles using a defect- decorated optical nanofber, H. Xin, B. Li, Opt Express. -2011. -Vol. 19(14). -P. 13285-13290.

Moradi, A. Strength control in multiple optical traps generated by means of diffrac- tive optical elements, $A$. Moradi, E. Ferrari, V Garbin, E. Di Fabrizio, D. Cojoc, Optoelectronics and Advanced Materials-Rapid Communications. -2007. -Vol. 1(4). -P. 158-161.

Emiliani, $V$ Multi force optical tweezers to generate gradients of forces, V Emiliani, D. Sanvitto, M. Zahid, F Gerbal, M. Coppey-Moisan, Opt Express. -2004. -Vol. 12(17). -P. 3906-3910.

Dasgupta, R. Optical orientation and rotation of trapped red blood cells with Laguerre-Gaussian mode, R. Dasgupta, S. Ahlawat, R.S. Verma, PK. Gupta, Opt Express. -2011. -Vol. 19(8). -P. 7680-7685.

Daria, VR. Simultaneous transfer of linear and orbital angular momentum to mul- tiple low-index particles, VR. Daria, M.A. Go, H.-A. Bachor, Journal of Optics. - 2011. -Vol. 13(4). -P. 044004.

Sun, Q. Hollow sinh-Gaussian beams and their paraxial properties, Q. Sun, K. Zhou, G. Fang, G. Zhang, Z. Liu, S. Liu, Opt Express. -2012. . Vol. 20(9). -P. 9682-9691.

Olson, C.C. Tailored optical force felds using evolutionary algorithms, C.C. Olson, R.T Schermer, F Bucholtz, Opt Express. -2011. -Vol. 19(19). -P. 18543-18557.

Jesacher, A. Size selective trapping with optical "cogwheel" tweezers, A. Jesacher, S. Fürhapter, S. Bernet, M. Ritsch-Marte, Opt Express. -2004. -Vol. 12(17). -P. 4129-4135.

Brijesh, P Spatially shaping the longitudinal focal distribution into a horseshoe- shaped profle, $\mathrm{P}$ Brijesh, $\mathrm{T}$. J. Kessler, J. D. Zuegel, D. D. Meyerhofer, Quantum Electronics and Laser Science Conference. -2007. QELS '07 (2007). -P. 1-2.

Jiang, Y Radiation force ofhighly focused Lorentz-Gauss beams on a Rayleigh par- ticle, Y Jiang, K. Huang, X. Lu, Opt Express. -2011. -Vol. 19(10). -P. 97089713.

Steuernagel, O. Coherent Transport and Concentration of Particles in Optical Traps using Varying Transverse Beam Profles, O. Steuernagel, arXiv:physics/0502023-2005.

Volostnikov VG., et al., Kvant. Elektronika. -2002. -V. 32, No. 7. -565-566.

Abramochkin E.G., et al., Izv. RAN. Ser. Fiz. -2008. -V. 72, No. 1. -C. 76-79.

Bowman, R. Particle tracking stereomicroscopy in optical tweezers: Control oftrap shape, R. Bowman, G. Gibson, M. Padgett, Opt. Express. -2010. -Vol. 18(11). -P. 11785-11790.

Saito, T.L. SCMD: Saccharomyces cerevisiae Morphological Database, T.L. Saito, M. Ohtani, H. Sawai, F Sano, A. Saka, D. Watanabe, M. Yukawa, Y Ohya, S. Mor- ishita, Nucleic Acids Res. -2004-Vol. 32 (Database issue) -P. D319-322. 
Simpson, S.H. Computational study of the optical trapping of ellipsoidal particles, S.H. Simpson, S. Hanna, Phys. Rev. A. -2011. -Vol. 84(5). -P. 053805.

Skidanov R.V, et al., Komp. Optika. -2010. -V 34, No. 3. -308-314.

Svoboda, K. Biological Applications of Optical Forces, K. Svoboda, S. M. Block, Annual Review of Biophysics and Biomolecular Structure. - 1994. -Vol. 23. -P. 247-285. 